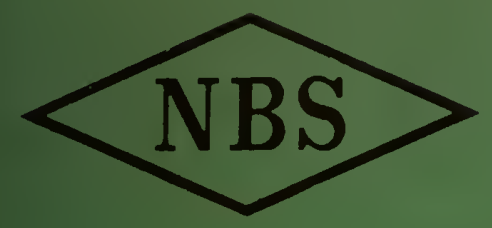

Eechnical Note

no. 314

\title{
STABILITY OF TWO-PHASE ANNULAR FLOW IN A VERTICAL PIPE
}

STEPHEN JARVIS, Jr.

U. S. DEPARTMENT OF COMMERCE NATIONAL BUREAU OF STANDARDS 


\section{THE NATIONAL BUREAU OF STANDARDS}

The National Bureau of Standards is a principal focal point in the Federal Government for assuring: maximum application of the physical and engineering sciences to the advancement of technology in industry and commerce. Its responsibilities include development and maintenance of the national standards of measurement, and the provisions of means for making measurements consistent with those standards; determination of physical constants and properties of materials; development of methods for testing materials, mechanisms, and structures, and making such tests as may be necessary, particularly for government agencies; cooperation in the establishment of standard practices for incorporation in codes and specifications; advisory service to government agencies on scientific and technical problems; invention and development of devices to serve special needs of the Government; assistance to industry, business, and consumers in the development and acceptance of commercial standards and simplified trade practice recommendations; administration of programs in cooperation with United States business groups and standards organizations for the development of international standards of practice; and maintenance of a clearinghouse for the collection and dissemination of scientific, technical, and engineering information. The scop? of the Bureau's activities is suggested in the following listing of its four Institutes and their organizational units.

Institute for Basic Standards. Applied Mathematics. Electricity. Metrology. Mechanics. Heat. Atomic Physics. Physical Chemistry. Laboratory Astrophysics. ${ }^{*}$ Radiation Physics. Radio Standards Laboratory: " Radio Standards Physics; Radio Standards Engineering. Office of Standard Reference Data.

Institute for Materials Research. Analytical Chemistry. Polymers. Metallurgy. Inorganic Materials. Reactor Radiations. Cryogenics.* Materials Evaluation Laboratory. Office of Standard Reference Materials.

Institute for Applied Technology. Building Research. Information Technology. Performance Test Development. Electronic Instrumentation. Textile and Apparel Technology Center. Technical Analysis. Office of Weights and Measures. Office of Engineering Standards. Office of Invention and Innovation. Office of Technical Resources. Clearinghouse for Federal Scientific and Technical Information. **

Central Radio Propagation Laboratory.* Ionospheric Telecommunications. Tropospheric Telecommunications. Space Environment Forecasting. Aeronomy.

* Located at Boulder, Colorado 80301

** Located at 5285 Port Royal Road, Springfield, Virginia 22171. 


\title{
NATIONAL BUREAU OF STANDARDS Eechnical Mote 314 ISSUED June 7, 1965
}

\author{
STABILITY OF TWO-PHASE ANNULAR FLOW \\ IN A VERTICAL PIPE
}

Stephen Jarvis, Jr.

National Bureau of Standards

Boulder, Colorado

NBS Technical Notes are designed to supplement the Bureau's regular publications program. They provide a means for making available scientific data that are of transient or limited interest. Technical Notes may be listed or referred to in the open literature. 



\section{CONTENTS}

Page

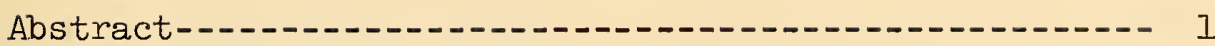

1. Introduction--...- I

2. The Model and Basic Equations-_. 3

3. Fundamental Solution System for Small Viscosity------- 8

4. Singular Point $r=0$

5. Critical Layer Solutions-- 18

6. The Eigenvalue Problem-_. 21

7. Base Flow-- 31

8. Numerical Computation-_- 34

9. Results-- 38

10. References-_- 41

11. Glossary- 42 Appendix 1- 45

Appendix 2-0 50

Appendix 3-0 55

Appendix 4- 58

Appendix 5- 63

Appendix 6-1.- 67

Figure 1-- 71

Figure 2- 72

Figure 3-- 73

Figure 4-1 74

Figure 5-_..- 75

Figure 6-- 76

Figure 7-0.- 77

Figure 8-0 78

Figure 9-_- 79

Figure 10-... 80

Table 1-.

Table 2---1- 82

Table 3-. 84 
Page

Table 4-1- 85

Table 5-1-1

Table 6---1

Table 7 -1-- 88 


\section{Stability of Two-Phase Annular Flow in a Vertical Pipe \\ Stephen Jarvis, Jr.}

The theory of hydrodynamic stability for infinitesimal disturbances is applied to the steady symmetric annular flow of two incompressible fluids in a vertical pipe. The resulting $12 \mathrm{x} 12$ complex determinant for the determination of curves of neutral stability is reduced, by suitable approximations, to an $8 \times 8$ one, and numerical methods are used to determine some neutral curves for air-water mixtures.

\section{Introduction}

In this report, an analysis is made of the stability of two-component annular flow in vertical tubes. The approach employs the well-known stability theory of infinitesimal disturbances [1].

Because of its importance in many engineering applications (e.g., fluid transport, heat exchangers), two-phase flow in tubes and orifices has received a great deal of attention in the last decade. But because the flow is very complex, even in straight vertical pipes, most of the effort has been devoted to accumulating a large quantity of experimental information, and to attempting to correlate data in particular cases with theories based on empirical relations. Furthermore, most of the work deals with the apparently simple (though important) case of annular flow without heat transfer or phase exchange; yet the results to date for this case are theoretically unsatisfactory and of modest practical utility. It is not the aim of this paper to correct this situation, but merely to apply the theory of hydrodynamic stability to this simple case in a more general way than has been used heretofore in this area. The stability theory has a record of significant (though not uniform) success.

Restricting ourselves to two-component incompressible flow in vertical pipes (e.g., air and water), the flows resulting experimentally are readily described in terms of "modes" dependent essentially on the ratio, of gas flow rate $Q_{g}$, to liquid flow rate $Q_{\ell}$. As $r$ increases from zero, we have several modes manifesting themselves in the following order:

A. Aerated flow: gas bubbles entrained in an otherwise normal liquid flow.

B. Piston flow: the gas collects into a train of regularly-shaped slugs which nearly span the pipe. 
C. Churn flow: a chaotic flow of large gas and liquid masses of irregular form.

D. Wave entrainment: the liquid lies against the pipe wall, while the gas fills the central core, the interface being marked by large waves which nearly span the pipe.

E. Annular flow: the large waves of $D$, damp to form a barely rippled surface on the liquid film.

F. Entrained flow: liquid droplets are torn from the film surface and carried along with the gas.

One is tempted to attribute to annular flow, E, a low- $\boldsymbol{\text { instability }}$ leading to wave entrainment, $D$, and a high-rinstability leading to droplet entrainment, F. There is, however, a conceptual difficulty in studying the stability of this annular flow via infinitesimal disturbances on an otherwise steady flow. The gas flow and liquid flows are usually turbulent in cases of interest, while the small interfacial ripples are known to be responsible (probably thru profile arag) for a significant part of the pressure drop. To what extent the small wavy disturbances admitted in the stability analysis can qualitatively or quantitatively approximate the experimentally observed flow can only be determined a posteriori. Some encouragement is given by the results of the only other stability study of two-phase flow known to the author, that of Feldman [2]. He considered the oversimplified model comprising two-dimensional film flow on a flat wall, with a linear velocity profile in both the film and the semiinfinite gas flow above it. While the critical Reynolds number determined was very poor, the destabilizing effects of surface tension and gravity (normal to the wall) were demonstrated. The most important result, however, was the existence of instability, although for a homogeneous flow with linear velocity profile (Couette flow), the analysis is known to predict stability for all Reynolds numbers (contrary to the physical facts however!). The instability must therefore be associated with the interface [3] rather than with the inception of turbulence in either fluid. However, Feldman appears to have erred in his formulation of the interface conditions, which may cause the failure of his result to match film cooling Reynolds numbers. 
The results obtained here, when reduced to Feldman's case, give very much lower critical Reynolds numbers, and agree in an acceptable manner with film data of Charvonia ${ }^{[4]}$.

Squire's result [8] for homogeneous two-dimensional flow, which states that three-dimensional infinitesimal disturbances are more stable than two-dimensional ones, is not clearly applicable to Feldman's model, and is not applicable to the model considered here. (Squire's result appears to fail for finite aisturbances.)

2. The Model and Basic Equations

The model chosen for this analysis. is that of vertical annular flow of two incompressible fluids in a circular pipe of radius $L$. The core fluid is referred to as the gas, the film fluid is referred to as the liquid. Gravity is assumed to act downward, in the $(-z)$ - direction. An undisturbed flow

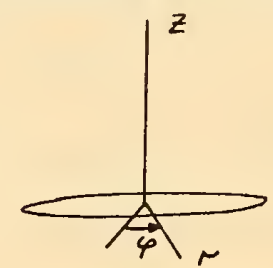
is assumed to exist with cylindrical symmetry having only a vertical velocity component $W(r)$. We assume $W$ is continuous, $W(I)=0$, and $W^{\prime}(r)<0$ for $0<r \leq L$. This latter assumption simplifies the analysis by permitting, at most, one critical layer while placing a limitation on the influence of gravity which is realized in most flows of practical interest. The pressure drop $\gamma=-\frac{d P}{d z}$ is assumed constant. In the gas and liquid, respectively, we have the pressures:

$$
\begin{aligned}
& P_{g}=-r z+P_{g_{0}} \\
& P_{l}=-r z+P_{l_{0}}
\end{aligned}
$$

The constant pressure difference is associated with surface tension at the interface

$$
\Delta P \equiv P_{\circ g}-P_{o l}=\frac{\sigma^{*}}{x_{\star}}
$$

where $x_{*}$ is the undisturbed interface radius and $\sigma^{*}$ the coefficient of surface tension. While the base flow $W(r)$ is assumed to satisfy the NavierStokes equations in each medium, more general flows will be considered; they are discussed in Section 7 . The remainder of the analysis requires 
no further specification of the base flow. On this base flow are superimposed small disturbances to the velocities (radial, azimuthal and axial, respectively) and the pressure of the form:

$$
\left(\begin{array}{l}
u(r) \\
v(r) \\
w(r) \\
\pi(r)
\end{array}\right) e^{i \alpha_{\star}\left(z^{*}-c_{\pi} t^{*}\right)+i m \varphi}
$$

whose amplitudes are taken to be sufficiently small that the linearized equations of flow suffice for their determination. Only real parts are taken to be physically significant. The longitudinal wave number is $\alpha_{*}, m$ gives the rotational mode, and $c_{x_{R}}$ is the wave speed: thus $c_{\star}=c_{x_{R}}+i c_{x I}$. The sign of $c_{* I}$ determines whether the disturbance will grow $\left(c_{\star I}>0\right)$ or decay $\left(c_{\star I}<0\right)$. When $\left(\alpha_{\star}, m\right)$ are given, $c_{*}$ is to be determined from the analysis.

From the Navier-Stokes equations in cylindrical coordinates, [5] we obtain the following ordinary differential equations by linearization:

$$
\begin{aligned}
& A u+\pi^{\prime}=\mu\left\{\begin{array}{c}
u^{\prime \prime}+\frac{1}{r} u^{\prime}-\frac{m^{2}+1}{r^{2} u} \\
-\frac{2 i m}{r^{2}} r-\alpha^{2} u
\end{array}\right\} \\
& A v+\frac{i m}{r} \pi=\mu\left\{\begin{array}{l}
v^{\prime \prime}+\frac{1}{r} v^{\prime}-\frac{m^{2}+1}{r^{2}} v \\
+\frac{2 i m}{r^{2}} u-\alpha^{2} v
\end{array}\right\} \\
& A_{w} w W^{\prime} 4+i \alpha_{*} \pi=\mu\left\{\begin{array}{l}
w^{\prime \prime}+\frac{1}{r} w^{\prime} \\
-\frac{m^{2}}{r^{2}} w-\alpha_{*}^{2} w
\end{array}\right\} \\
& u^{\prime}+\frac{1}{r} u+\frac{i m}{r} v+i \alpha_{\star} w=0 \\
& A(r) \equiv i \alpha_{*} p\left(W(r)-c_{*}\right)
\end{aligned}
$$

4 
where $(\rho, \mu)$ must be given their respective values in the film and in the core.

At the center of the pipe, $r=0$, we require the velocity components to be bounded. At the wall, $r=I$, the disturbance components must vanish:

$$
\begin{aligned}
& u(0), w(0), w(0) \quad \text { bounded } \\
& u(L)=v(L)=w(L)=0 .
\end{aligned}
$$

We assume the interface to be disturbed in the same way as the base flow, and put for the interface

$$
f(z, r, \varphi, t) \equiv r-x_{\star}+F_{0} e^{i \alpha_{*}\left(z-c_{x} t^{*}\right)+i m \varphi}=0,
$$

where again $\left|F_{0}\right|<<1$ is assumed. Since on the interface, with V/ the complete velocity vector,

$$
\frac{\partial f}{\partial t}+V / \nabla f=0
$$

we find, using $W_{x}=W\left(x_{\star}\right)$,

$$
F_{0}=+\frac{u\left(x_{x}\right)}{i \alpha_{x}\left(W_{x}-c_{x}\right)} .
$$

Now across the interface, $u, v, w+w$ must be continuous for this viscous flow. The remaining physical conditions are that the total shearing stress be continuous across the interface, while the total normal stress jump, $\Delta \sigma_{n}$, be given in terms of the mean curvature of the interface

$$
\Delta \sigma_{n}=\sigma^{\star} K_{m},
$$

where $\sigma^{\star}$ is again the surface tension coefficient. The base flow has the normal stress

$$
\sigma_{r r}^{0}=-P_{0}+r z
$$

and the shear stress $\sigma_{r z}^{0}=\tau_{0}=\mu W^{\prime}(r)$ for laminar flow. The complete stress tensor is then 


$$
\begin{aligned}
\sigma_{r r} & =\sigma_{r r}^{0}+\left(-\pi+2 \mu u^{\prime}\right) E_{0} \\
\sigma_{r \varphi} & =\sigma_{\varphi r}=\mu\left(r\left(\frac{\tilde{r}}{r}\right)^{\prime}+\frac{i m}{r} u\right) E_{0} \\
\sigma_{r z} & =\sigma_{z r}=\tau_{0}+\mu\left(i \alpha_{\star} u+w^{\prime}\right) E_{0} \\
\sigma_{\varphi z} & =\sigma_{z \varphi}=\mu\left(i \alpha_{\star} v+\frac{i m}{r} \omega\right) E_{0} \\
\sigma_{\varphi \varphi} & =\sigma_{r r}^{0}+\left(-\pi+2 \mu\left(\frac{i m}{r} v+\frac{1}{r} u\right)\right) E_{0} \\
\sigma_{z z} & =\sigma_{r r}+\left(-\pi+2 \mu i \alpha_{\star} \omega\right) E_{0} \\
E_{0} & =e^{i \alpha_{*}\left(z^{*}-c_{*} t^{*}\right)+i m \varphi}
\end{aligned}
$$

The unit outward normal to the interface has the components

$$
n_{j}=\left(1, n_{\varphi}, n_{z}\right)
$$

where $\left|n_{y}\right|,\left|n_{z}\right| \ll 1$, where:

$$
\begin{aligned}
& n_{\varphi}=-\frac{i m}{r} F_{0} E_{0} \\
& n_{z}=-i \alpha_{\star} F_{0} E_{0} .
\end{aligned}
$$

Thus the force on the interface has the components (neglecting quadratic terms in the disturbances):

$$
\left(\begin{array}{l}
\sigma_{r r}^{0}+\left(-\pi+2 \mu u^{\prime}-i \alpha_{*} F_{0} \tau_{0}\right) E_{0} \\
\left(u r\left(\frac{v}{r}\right)^{\prime}+\mu \frac{i m}{r} u-\frac{i m}{r} F_{0} \sigma_{r r}^{0}\right) E_{0} \\
\tau_{0}+\left(\mu i \alpha_{\pi} u+\mu \omega^{\prime}-i \alpha_{*} F_{0} \sigma_{r r}^{0}\right) E_{0}
\end{array}\right) .
$$

Now a tedious calculation of the mean curvature $K_{m}$ of the interface gives

$$
K_{m}=-\frac{1}{x_{*}}-\left(\alpha_{*}^{2}+\frac{m^{2}-1}{x_{*}^{2}}\right) E_{0} F_{0}
$$

to the first order in small quantities. (In this calculation, only the real part of $f$ is used, leading to $K_{m}$ which is the real part of (13).)

6 
Noting that on the undisturbed interface:

$$
\begin{aligned}
& \left.\sigma_{r r}^{0}\right|_{\text {liquid }} ^{\text {gas }}=-\frac{\sigma^{*}}{x_{\star}} \\
& \left.\tau_{0}\right|_{\text {liquid }} ^{\text {gas }}=0
\end{aligned}
$$

for the base flow, and putting

$$
\left[\tau_{0}(r)\right]_{f=0}=\tau_{0}\left(x_{\star}\right)+\tau_{0}^{\prime}\left(x_{\star}\right) F_{0} E_{0}
$$

to first order, we determine the jump conditions for the disturbances. In the following $\delta A=\left.A\right|_{\text {liquid }} ^{\text {gas }}$ and $r=x_{\star}$ :

$$
\begin{aligned}
& \delta u=\delta v=0, \quad \delta w+\frac{u\left(x_{*}\right)}{i \alpha_{\star} W_{x}-c_{\star}} \delta W_{x}^{\prime}=0 \\
& \left(2 \delta\left(\mu \omega^{\prime}\right)-\delta \pi=\frac{-i u \sigma^{*}}{\alpha_{\star}\left(W_{x}-c_{\star}\right)}\left(\alpha_{*}^{2}+\frac{m^{2}-1}{x_{*}^{2}}\right)\right. \\
& \left\{\delta\left(u v^{\prime}\right)+\frac{i m u-v}{x_{\star}} \delta \mu=\frac{\sigma^{\star} m u}{\alpha_{x} x_{x}^{2}\left(W_{x}-c_{\star}\right)}\right. \\
& \left(\delta\left(\mu w^{\prime}\right)+i \alpha_{\star} 4 \delta \mu+\frac{u}{i \alpha_{\star}\left(w_{x}-c_{\star}\right)}=\frac{\sigma^{\star} u}{x_{\star}\left(w_{x}-c_{\star}\right)}\right.
\end{aligned}
$$

The twelve conditions ( $6,7,15$ and 16$)$ are homogeneous in ( $u, v, w, \pi)$ as are the pair of 6 th order systems $(2-5)$, associated with the film and core flows. This will be seen in section 6 to lead to an eigenvalue problem for the determination of $c_{*}$ as a function of the other parameters of the problem. In fact, we shall be led to require that a complex determinant $\Delta=\Delta_{R}+i \Delta_{I}$ vanish:

$$
\left\{\begin{array}{l}
\Delta_{R}=0 \\
\Delta_{I}=0
\end{array}\right.
$$

We shall, in effect, set $C_{x}=0$, and eliminate $C_{\star R}$ between these real equations, obtaining a single relation among all the parameters $\alpha_{*}, \mu$, etc., of the problem which when satisfied, assures that the solutions so 
specified will be neutrally stable, that is, they will neither grow nor decay. This hypersurface in the space of the parameters will, in general, separate a region of stable solutions from a region of unstable solutions.

We shall now proceed to the details of determining solutions to the system $(2-5)$, bearing in mind that $c_{\star}$ will be taken to be real.

3. Fundamental Solution System for Small Viscosity

The customary approach to solving the complicated set of $(2-5)$ is to assume that the viscosity $\mu \rightarrow 0$. The resulting solutions can be proved to be rigorously asymptotic in domains devoid of the singularities of the solutions, i.e., $r=0$ and $r=r_{c}$, where $W\left(r_{c}\right)=c_{\star}$ (if such exists), the so-called "critical layer". We shall require that when $r_{c}$ exists for real $c_{*}$,

$$
r_{c} \neq\left(0, x_{\star}, L\right)
$$

Setting $\mu=0$, the system $(2-5)$ may be reduced to the system:

$$
\begin{aligned}
& \pi^{\prime \prime}+\left(\frac{1}{r}-\frac{2 W^{\prime}}{W-c_{*}}\right) \pi^{\prime}-\left(\frac{m^{2}}{r^{2}}+\alpha_{\star}^{2}\right) \pi=0 \\
& u=-\frac{\pi^{\prime}}{A} \\
& v=-\frac{i m}{r A} \pi \\
& w=-\frac{i \alpha_{m}}{A}+\frac{\rho W^{\prime}}{A^{2}} \pi^{\prime}
\end{aligned}
$$

This system is evidently of 2 nd order, and are the so-called "inviscid equations". They provide only two of the independent set of 6 solutions we must obtain for $(2-5)$ in order to be able to satisfy general boundary conditions. Before proceeding to the development of a complementary set of four solutions, we shall establish certain facts about the solutions of (17). The points $r=0$ and $r=r_{c}$ are regular singular points of (17). 
It does not appear possible to integrate these equations in closed form for general values of the parameters $m$ and $\alpha_{*}$ for the profiles $W(r)$ of interest. In studying the stability of plane boundary layer flow, the simpler equation $\pi^{\prime \prime}-\frac{2 W^{\prime}}{W-c} \pi^{\prime}-\alpha_{*}^{2} \pi=0$ is obtained which can be integrated in terms of hypergeometric functions for a useful profile. On the other hand, it turns out that the neutral stability curve is associated with $\alpha_{*} L^{*} \ll 1$ (where $L^{*}$ is the boundary layer thickness), so that the solutions may be determined adequately by two terms of an expansion in $\alpha_{*}^{2}$. In the present case, we could solve the equation in terms of (untabulated) hypergeometric functions for $\alpha_{\star} L \rightarrow 0$, but Feldman's results indicate the neutral stability curve involves values $\alpha_{\star}$ between the values $0.01 \leqslant \alpha_{\star}\left(L-x_{\star}\right) \leqslant 1$, so that we must be prepared to have wave numbers of the order of the inverse film thickness, too large for this approximation.

When a critical layer occurs in the flow at $r=r_{c}$, it will be apparent from Section 5 that certain phases must be adopted to continue properly asymptotic solutions across the singularity:

$$
\begin{aligned}
& 0 \leqslant \arg \left(r-r_{c}\right) \leqslant \pi \\
& \pi \leqslant \arg (W-c) \leqslant 2 \pi \\
& \frac{3 \pi}{2} \leqslant \arg A \leqslant \frac{5 \pi}{2} \\
& \arg t=\frac{3 \pi}{2} \\
& \frac{\pi}{2} \leqslant \arg \zeta \leqslant \frac{3 \pi}{2},
\end{aligned}
$$

where

$$
t \equiv \frac{d A}{d r}, \quad \quad \rho \equiv \mu^{-1 / 3} t^{-2 / 3} A .
$$

It is convenient to define here specific solutions of the inviscid equation which will be used in the numerical work. For this we shall 
introduce the dimensionless quantities:

$$
\begin{array}{ll}
\xi=r / L, & \alpha=\alpha_{*} L, \\
x=x_{*} / L, & c=c_{*} / W_{x},
\end{array}
$$

and put

$$
\pi(r)=\pi(\xi), \quad Q(\xi)=\pi^{\prime}(\xi) \text {. }
$$

The inviscid equations for $(\pi, Q)$ are then

$$
\left\{\begin{array}{l}
T^{\prime}=Q \\
Q^{\prime}=\left(\frac{m^{2}}{\xi^{2}}+\alpha^{2}\right) \pi+\left(\frac{2 r^{\prime}}{m-c}-\frac{1}{\xi}\right) Q .
\end{array}\right.
$$

We define a pair of independent solutions of (22) in the liquid film by their values at the (assumed regular) point $\xi=x$ :

$$
\left\{\begin{array}{l}
\left(T_{5}(\xi), Q_{5}(\xi)\right)_{\xi=x}=(1,0) \\
\left(\pi_{6}(\xi), Q_{6}(\xi)\right)_{f=x}=(0,1) .
\end{array}\right.
$$

Their values at the wall $\xi=l(r=L)$ will be denoted by $\left(\prod_{j L}, Q_{j L}\right.$; $j=5,6$ ) which may be complex.

In the gas core, we shall define a unique $H \equiv \xi \frac{Q_{2}(\xi)}{\pi_{2}(\rho)}$ by the requirement (since $r_{c} \neq 0$ ):

$$
\pi_{2}(\xi) \underset{\xi \rightarrow 0}{\longrightarrow} \xi^{m}
$$

(Any linearly independent solution $T_{1}(\rho)$ is singular at $\xi=0$. ) In Section 8, we shall assume a core flow of the form:

$$
W(\xi)=k-f \xi^{2} \text {. }
$$

Then with

$$
\bar{\sigma}=\alpha \xi, \quad \bar{\beta}=\alpha\left(\frac{k-c}{f}\right)^{1 / 2}
$$

10 
$H(\bar{\sigma} ; \bar{\beta} ; m) \quad$ satisfies the equations

$$
\left\{\begin{array}{c}
H(\bar{\sigma}) \equiv H_{R}(\bar{\sigma})+i H_{I}(\bar{\sigma}) \\
\bar{\sigma} H_{R}^{1}+H_{R}^{2}-H_{I}^{2}+\frac{4 \bar{\sigma}^{2}}{\bar{\beta}^{2}-\bar{\sigma}^{2}} H_{R}-\left(m^{2}+\bar{\sigma}^{2}\right)=0 \\
\bar{\sigma} H_{I}^{\prime}+2 H_{R} H_{I}+\frac{4 \bar{\sigma}^{2}}{\bar{\beta}^{2}-\bar{\sigma}^{2}} H_{I}=0
\end{array}\right.
$$

for real and imaginary parts. The initial condition for $\mathrm{H}$ is:

$$
\left\{\begin{array}{l}
H_{I}(0)=0 \\
H_{R}(\bar{\sigma}) \underset{\bar{\sigma} \rightarrow 0}{\longrightarrow} m+\frac{\bar{\sigma}^{2}}{2(1+m)}\left(1-\frac{4 m}{\bar{\beta}^{2}}\right) \\
+\theta\left(\bar{\sigma}^{+}\right) .
\end{array}\right.
$$

The critical layer is at $\bar{\sigma}=\bar{\sigma}_{c}=\bar{\beta}$. Of course, $H_{I}(\bar{\sigma}) \equiv 0$ for $\bar{\sigma}<\bar{\beta}$.

We now turn to the derivation of a complementary set of solutions for $(2-5)$. The approach is based on the results of characteristic theory for inviscid flows, in which jumps in tangential velocity across streamlines are permitted. Then for small viscosity, we expect derivatives across the base flow streamlines ( $y=$ constant) to be large. We set:

$$
\left(\begin{array}{l}
u \\
v \\
w \\
\pi
\end{array}\right)=\left(\begin{array}{c}
\mu^{1 / 2} u_{0}+u_{1}+\cdots \\
v_{0}+\mu^{1 / 2} v_{1}+\cdots \\
w_{0}+\mu^{1 / 2} w_{1}+\cdots \\
\mu^{3 / 2} \pi_{0}+\mu^{2} \pi_{1}+\cdots
\end{array}\right) e^{\mu^{-1 / 2} \int_{x_{\star}}^{r} d r g(r) .}
$$

Substituting these in (2-5), and ordering in powers

11 
of $\mu^{1 / 2}$, the leading terms in each equation are homogeneous algebraic equations in $\left(u_{0}, U_{0}, w_{0}, \pi_{0}\right)$. For solutions to exist, the determinant of the coefficients must vanish. This leads to two determinations of $g(r):$

$$
\left\{\begin{array}{l}
g_{1}(r)=A^{1 / 2} \\
g_{2}(r)=A^{1 / 2} e^{-i \pi}
\end{array}\right.
$$

The next highest order set of equations leads, for each $g$, to two independent list order differential systems in $\left(u_{0}, v_{0}, w_{0}, \pi_{0}\right)$ :

$$
\left\{\begin{array}{l}
2 r^{2} g v_{0}^{\prime}+r^{2} v_{0} g^{\prime}+r g U_{0}=0 \\
2 r^{2} g W_{0}^{\prime}+r^{2} W_{0} g^{\prime}+r g w_{0}-p r^{2} W^{\prime} u_{0}=0
\end{array}\right.
$$

with the auxiliary specifications:

$$
\left\{\begin{array}{l}
u_{0}=-\frac{1}{r g}\left(i m v_{0}+i \alpha_{x} r w_{0}\right) \\
\pi_{0}=\frac{2}{(r g)^{1 / 2}}\left((r g)^{1 / 2} u_{0}\right)^{\prime}-\frac{2 i m}{r^{2} g} v_{0} .
\end{array}\right.
$$

Equations 25 and 26 together with the solutions of (17) enable us to write the general solution in the gas core:

$$
\begin{aligned}
& v=\frac{C_{1} e_{1}}{\left(r^{2} A\right)^{1 / 4}}+\frac{i C_{2} e_{2}}{\left(r^{2} A\right)^{1 / 4}}+C_{5} v_{1}+C_{6} v_{2} \\
& w=-\frac{m \int_{x^{*}}^{r} \frac{d r}{r} \frac{W^{\prime}}{\left(W-C_{x}\right)^{1 / 2}}}{2 \alpha_{*}\left(W-c_{*}\right)^{1 / 2}\left(r^{2} A\right)^{1 / 4}}\left(C_{1} e_{1}+i C_{2} e_{2}\right) \\
& +\frac{1}{\left(r^{2} A\right)^{1 / 4}\left(W-C_{*}\right)^{1 / 2}}\left(C_{3} e_{1}+i C_{+} e_{2}\right) \\
& +C_{5} w_{1}+C_{6} w_{2} \\
& u=-\frac{i \alpha_{\star} r \mu^{1 / 2}}{\left(W-c_{*}\right)^{1 / 2}\left(r^{2} A\right)^{3 / 4}}\left(C_{3} e_{1}-i C_{4} e_{2}\right) \\
& -\frac{i m \mu^{1 / 2}}{\left(r^{2} A\right)^{3 / 4}}\left(1-\frac{\frac{1}{2} r}{\left(W-c_{*}\right)^{1 / 2}} \int_{x_{*}}^{r} \frac{d r}{r} \frac{W^{\prime}}{\left(W-c_{*}\right)^{1 / 2}}\right)\left(C_{1} e_{1}-i C_{2} e_{2}\right) \\
& +\mathrm{C}_{5} \mathrm{H}_{1}+\mathrm{C}_{6} \mathrm{u}_{2}
\end{aligned}
$$

12 


$$
\begin{aligned}
\pi=C_{5} \pi & +C_{6} \pi_{2} \\
& +\mu^{3 / 2} L\left(C_{1}, C_{2}, C_{3}, C_{4}\right) .
\end{aligned}
$$

(29)

Here

$$
e_{1,2}=\exp \left\{ \pm \mu^{-1 / 2} \int_{x^{\prime}}^{r} d r A^{1 / 2}\right\} \text {. }
$$

The latter term in $\pi$ need not be explicitly given. A similar solusion can be written for the liquid film.

Clearly, the solutions (29) are singular at $r=\left(0, r_{c}\right)$, but the conditions (18) determine their correct continuations.

However, since boundary conditions on the general solution must be applied at $r=\left(0, x_{*}, L\right)$, it is desirable to derive solutions which are regular at these points without restricting a priori the location of the critical layer. Furthermore, this analysis will determine the continuation conditions (18).

For convenience later.in joining solutions, we shall show how the solutions (29) behave as $r \rightarrow 0$ and $r \rightarrow x_{\star}$.

$\stackrel{r \rightarrow 0}{ }$

$$
\begin{aligned}
& \text { Writing } A(0)=A_{0} \text {, and } \\
& \begin{aligned}
E_{1,2} & =e^{ \pm \mu^{-1 / 2} A_{0}^{1 / 2} r} \\
P_{0} & =e^{-\mu^{-1 / 2} \int_{0}^{x_{*}} d r A^{1 / 2}} \\
P_{1} & =-\int_{0}^{x_{*}} d r \frac{1}{r} \frac{W^{1}}{\left(W-c_{*}\right)^{1 / 2}}
\end{aligned}
\end{aligned}
$$

we find as $r \rightarrow 0$ :

13 


$$
\begin{aligned}
v \rightarrow & \frac{C_{1} p_{0}}{A_{0}^{1 / 4}} \frac{E_{1}}{r^{1 / 2}}+\frac{i C_{2}}{p_{0} A_{0}^{1 / 4}} \frac{E_{2}}{r^{1 / 2}}+C_{5} v_{1}+C_{6} v_{2} \\
w \rightarrow & \frac{-m p_{1}}{2 \alpha_{4}\left(W_{0}-C_{*}\right)^{1 / 2} A_{0}^{1 / 4}}\left\{C_{1} p_{0} \frac{E_{1}}{r^{1 / 2}}+i C_{2} \frac{E_{2}}{p_{0} r^{1 / 2}}\right\} \\
& +\frac{1}{A_{0}^{1 / 4}\left(W_{0}-C_{*}\right)^{1 / 2}}\left\{C_{3} p_{0} \frac{E_{1}}{r^{1 / 2}}+i C_{4} \frac{E_{2}}{p_{0} r^{1 / 2}}\right\} \\
& +C_{5} w_{1}+C_{6} \omega_{2} \\
u \rightarrow & \frac{-i \alpha_{*} \mu^{1 / 2}}{A_{0}^{3 / 4}\left(W_{0}-C_{*}\right)^{1 / 2}}\left\{C_{3} p_{0} \frac{E_{1}}{r^{1 / 2}}-i C_{4} \frac{E_{2}}{p_{0} r^{1 / 2}}\right\} \\
& -\frac{i m \mu^{1 / 2}}{A_{0}^{3 / 4}}\left\{C_{1} p_{0} \frac{E_{1}}{r^{3 / 2}}-i C_{2} \frac{E_{2}}{p_{0} r^{3 / 2}}\right\} \\
& +C_{5} u_{1}+C_{6} u_{2} \\
\longrightarrow & C_{5} \pi_{1}+C_{6} \pi_{2}+L \mu^{3 / 2}
\end{aligned}
$$

$$
\begin{aligned}
& \underset{\text { Writing }}{r \rightarrow x_{\star}:} \\
& \begin{array}{l}
A \rightarrow p+t\left(r-x_{*}\right), \\
\zeta=A \mu^{-1 / 3} t^{-2 / 3},
\end{array} \quad\left\{\begin{array}{l}
t \equiv i \alpha_{*} \rho W_{x}^{\prime} \\
p=A\left(x_{*}\right) \neq 0
\end{array}\right. \\
& E_{3,4}=e^{ \pm \frac{2}{3} r^{3 / 2}} \\
& P_{2}=e^{-\frac{2}{3} \mu^{-1 / 2} t^{-1} p^{3 / 2}}
\end{aligned}
$$

14 
Then as $r \rightarrow X_{\star}:$

$$
\begin{aligned}
& v \rightarrow\left(u^{1 / 3} t^{2 / 3} x_{*}^{2}\right)^{-1 / 4}\left(\frac{C_{1} E_{3} P_{2}}{\rho^{1 / 4}}+\frac{i C_{2} E_{4}}{P_{2} \rho^{1 / 4}}\right) \\
& +C_{5} v_{1}+C_{6} v_{2} \\
& w \rightarrow\left(x_{*} W_{x}^{1} \mu^{1 / 2}\right)^{-1 / 4}\left(\frac{C_{3} E_{3} P_{2}}{S^{3 / 4}}+\frac{i C_{4} E_{4}}{P_{2} e^{3 / 4}}\right) \\
& +C_{5} w_{1}+C_{6} w_{2} \\
& u \rightarrow \frac{-i \alpha_{*} \mu^{1 / 12}}{\left(x_{*} W_{x}^{1} t^{2 / 3}\right)^{1 / 2}}\left(\frac{C_{3} E_{3} P_{2}}{\rho^{5 / 4}}-\frac{i C_{4} E_{4}}{P_{2} S^{5 / 4}}\right) \\
& -\frac{i m \mu^{1 / 2}}{\left(x^{6} \mu t^{2}\right)^{1 / 4}}\left(\frac{C_{1} E_{3 P_{2}}}{\rho^{3 / 4}}-\frac{i C_{2} E_{4}}{p_{2} \rho^{3 / 4}}\right) \\
& +C_{5} u_{1}+C_{6} u_{2} \\
& \pi \rightarrow C_{5} \pi_{1}+C_{6} \pi_{2}+\mu^{3 / 2} L .
\end{aligned}
$$

4. Singular Point $\underline{r=0}$.

Regular solutions to the system (2-5) may be obtained by introduping the singular - layer coordinate:

$$
z=r \mu^{-1 / 2}
$$

and expanding the dependent variables and coefficients in the equations

15 
in powers of $\mu^{1 / 2}$ :

$$
\begin{aligned}
& u=u_{0}(z)+\cdots \\
& v=v_{0}(z)+\cdots \\
& w=\mu^{1 / 2} W_{0}(z)+\cdots \\
& \pi=\mu^{1 / 2} \pi_{0}(z)+\cdots \\
& W=W_{0}+\sigma\left(r^{2}\right) .
\end{aligned}
$$

Substituting in $(2-5)$, one can derive a single factorable 4 th order equation in $\mathrm{Z}_{0}$.

Putting $\quad D^{0} \equiv \frac{d}{d z}$ :

$$
\begin{aligned}
& {\left[D^{2}+\frac{3}{3} D+\left(\frac{1-m^{2}}{z^{2}}-A_{0}\right)\right] Q=0} \\
& {\left[D^{2}+\frac{3}{3} D+\frac{1-m^{2}}{z^{2}}\right] U_{0}=Q .}
\end{aligned}
$$

The remaining variables are determined by the and order auxiliary system

$$
\begin{aligned}
& U_{0}=-\frac{1}{i m}\left(z U_{0}^{\prime}+U_{0}\right) \\
& \Gamma=\frac{z}{i m}\left[V_{0}^{\prime \prime}+\frac{1}{z} U_{0}^{\prime}-\left(\frac{1+m^{2}}{z^{2}}+A_{0}\right) U_{0}+\frac{2 i m}{z^{2}} U_{0}\right] \\
& {\left[D^{2}+\frac{1}{z} D-\left(\frac{m^{2}}{z^{2}}+A_{0}\right)\right] r_{0}=i \alpha \pi \tau_{0}+\rho W_{0}^{\prime \prime} z U_{0} .}
\end{aligned}
$$

These equations are readily solved in terms of Bessel functions. In applying the regularity conditions at $r=0$, we must distinguish the cases $m=0$ and $m \neq 0$. The required solutions are as follows:

$$
\begin{aligned}
u_{0}= & B_{2} z^{-1+m} \\
& +B_{3}\left\{\begin{array}{c}
z^{-1-m} \int_{0}^{z} d \rho \Gamma^{1+m} I_{m}\left(5 A_{0}^{1 / 2}\right) \\
-z^{-1+m} \int_{0}^{z} d \Gamma \Gamma^{1-m} I_{m}\left(5 A_{0}^{1 / 2}\right)
\end{array}\right\} \\
W_{0}= & B_{5} I_{m}\left(z A_{0}^{1 / 2}\right)+\hat{L}\left(B_{2}, B_{3}\right)
\end{aligned}
$$

(32)

16 
the latter term depending on $\boldsymbol{U}_{0}$.

$$
\begin{aligned}
& V_{0}=B_{1} I_{1}\left(z A_{0}^{1 / 2}\right) \\
& W_{0}=B_{3} I_{0}\left(z A_{0}^{1 / 2}\right) \\
& u_{0}=-i \alpha_{x} z \int_{0}^{z} d s w_{0}\left(\rho A_{0}^{1 / 2}\right) \\
& (m=0) \\
& \pi_{0}=\bar{B}_{5}+\int_{0}^{z} d r\left[u_{0}^{\prime \prime}+\frac{1}{p} u_{0}^{\prime}-\left(A_{0}+\frac{1}{\sigma^{2}}\right) u_{0}\right] .
\end{aligned}
$$

From the known asymptotic behavior of the Bessel functions, one can deduce forms for these solutions as $\left|z A_{0}^{1 / 2}\right| \rightarrow \infty$. These are matched with the corresponding asymptotic solutions evaluated as $r \rightarrow 0$ (30), and relations between the constants $B_{j}, C_{k}$-are found. Elimination of the $B_{j}$ gives three relations among the $C_{k}$ required for regularity of the solutions on the axis. Details of this procedure are given in Aspendix 3 where the notation is defined. We obtain the following:

$$
\begin{aligned}
& \left\{\begin{array}{l}
C_{2}-C_{1} P_{0}^{2}=0 \\
C_{4}+C_{3} P_{0}^{2}=0 \\
C_{5}-\frac{i \alpha_{*} \sqrt{2 \pi} \mu^{3 / 4} P_{0} i_{01}}{\left(W_{0}-C_{*}\right)^{1 / 2}} C_{3}=0
\end{array} \quad(m=0) \quad(34)\right. \\
& \left\{\begin{array}{l}
C_{2}-\frac{\gamma^{*}}{r^{*}} i P_{0}^{2} C_{1}=0 \\
C_{4}+P_{0}^{2} e^{-i m \pi} C_{3}=0 \\
C_{5}+\frac{i m, 1+m}{r^{*}} i P_{0} \mu^{\frac{1+2 m}{4}} A_{0}^{\frac{1-m}{2}} C_{1}=0 .
\end{array} \quad(m \neq 0) \quad(35)\right.
\end{aligned}
$$

17 


\section{Critical Layer Solutions}

We shall now derive a set of solutions to the system (2-5) which are regular in a neighborhood of the singular point, yet are of the same character as the asymptotic solutions outside such a neighborhood.

We shall assume that in a neighborhood of a fixed (though arbitrary) point $r_{s}$,

$$
\begin{aligned}
& A=p+t\left(r-r_{s}\right)+\theta\left(\left(r-r_{s}\right)^{2}\right) \\
& W^{\prime}=W_{s}^{\prime}+\theta\left(r-r_{s}\right)=\frac{t}{i \alpha_{*} p}+\theta\left(r-r_{s}\right)
\end{aligned}
$$

and confine ourselves to a neighborhood of $r_{s}$ in which the higher order terms are negligible. If $r_{s}=r_{c}, P=0$; no restriction will be placed on the magnitude of $p$.

Iet

$$
C=\left(\mu t^{2}\right)^{-1 / 3} A
$$

Iet

$$
\left(\begin{array}{l}
w \\
w \\
u \\
\pi
\end{array}\right)=\left(\begin{array}{l}
q(5) \\
w(5) \\
u^{1 / 3} u(5) \\
\mu \pi(5)
\end{array}\right) .
$$

To the highest order in $\mu^{1 / 3}$, we obtain the 6th order system

$$
\left\{\begin{array}{l}
v^{\prime \prime}-r q=0 \\
u^{\prime \prime \prime}-r u^{\prime}+u=0 \\
w^{\prime}=-\frac{1}{i \alpha_{0}}\left(t^{\prime / 3} u^{\prime}+\frac{i m}{x_{*}} u\right) \\
\pi^{\prime}=t^{\prime / 3}\left(u^{\prime \prime}-r u\right) .
\end{array}\right.
$$

The first two do not depend on the geometry of the problem. They occur in stability analyses in general, and lead to the well-known Tietjen's function and related functions. 
Let

$$
\left\{\begin{array}{l}
Z_{k}=\int_{c_{1}} \frac{d t}{t^{k}} e^{\frac{1}{3} t^{3}-e t} \\
T_{k}=\int_{c_{2}} \frac{d t}{t} e^{\frac{1}{3} t^{3}-e t}
\end{array}\right.
$$

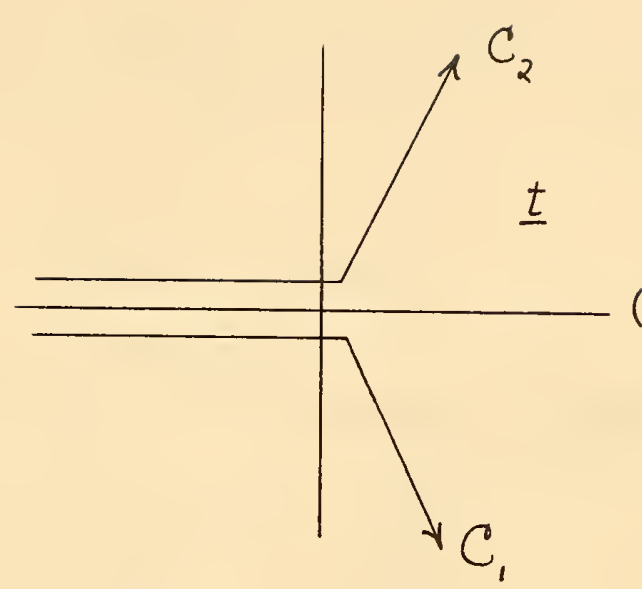

where the contour $C_{2}\left(\infty e^{i \pi}\right.$ to $\left.\infty e^{i \frac{\pi}{3}}\right)$ passes over $t=0$ and $C_{1}\left(\infty e^{-i \pi}\right.$ to $\left.\infty e^{-i \frac{\pi}{3}}\right)$ passes under $t=0$. We write the expressions:

$$
\begin{aligned}
& v=A_{1} Z_{0}+A_{2} Y_{0}+A_{5} v_{1}+A_{6} v_{2} \\
& u=A_{3} Z_{2}+A_{4} Y_{2}+A_{5} u_{1}+A_{6} u_{2} \\
& w=\frac{1}{i \alpha_{*}}\left(\frac{t}{\mu}\right)^{1 / 3}\left(A_{3} Z_{1}+A_{+} Y_{1}\right) \\
& -\frac{m}{\alpha_{*} x_{*}}\left(A_{1} Z_{0}+A_{2} \Psi_{0}\right)+A_{5} w_{1}+A_{6} w_{2} \\
& \pi=\left(\omega^{2} t\right)^{1 / 3}\left\{A_{3}\left(Z_{4}+r Z_{3}-Z_{1}\right)+A_{4}\left(\Psi_{4}+\rho Y_{3}-\Psi_{1}\right)\right\} \\
& +A_{5} \pi_{1}+A_{6} \pi_{2} \text {. }
\end{aligned}
$$

When $A_{5}=A_{6}=0$, this set satisfies (36). The inviscid solutions $\left(\pi_{1}, \pi_{2}\right)$ are appended as extensions to the approximations to the inviscid solutions which are obtained from the pole contributions of (37).

It is readily shown by the method of steepest descents that with the specification (18):

$$
\frac{\pi}{2} \leqslant \arg \rho \leqslant \frac{3 \pi}{2}
$$


we have the asymptotic evaluations:

$$
\begin{aligned}
& Z_{k}(r) \sim \sqrt{\pi}(-)^{k} e^{\frac{2}{3} e^{3 / 2}} e^{-\frac{1+2 k}{4}} \cdot \\
& \cdot\left[1+e^{-3 / 2}\left(\frac{5}{48}+\frac{k(k+2)}{4}\right)+\theta\left(r^{-3}\right)\right] \\
& Y_{k}(r) \sim i \sqrt{\pi} e^{-\frac{2}{3} r^{3 / 2}} e^{-\frac{1+2 k}{4}} \cdot \\
& \cdot\left[1-5^{-3 / 2}\left(\frac{5}{48}+\frac{k(k+2)}{4}\right)+\theta\left(r^{-3}\right)\right]
\end{aligned}
$$

Specifying $r_{s}=x_{*},\left(r \leqslant x_{*}\right)$ in (38), and using the asymptotic forms (39), we readily identify the coefficients $A_{j}$ with the $C_{j}$ of (31):

$$
\begin{aligned}
& \left(\begin{array}{l}
A_{1} \\
A_{2}
\end{array}\right)=\frac{1}{\sqrt{\pi}\left(\mu^{1 / 3} t^{2 / 3} x_{*}^{2}\right)^{1 / 4}}\left(\begin{array}{c}
p_{2} C_{1} \\
C_{2} / p_{2}
\end{array}\right) \\
& \left(\begin{array}{l}
A_{3} \\
A_{4}
\end{array}\right)=-\frac{i \alpha_{\star} \mu^{\frac{1}{12}}}{\sqrt{\pi}\left(x_{\star} W_{x}^{1} t^{2 / 3}\right)^{1 / 2}}\left(\begin{array}{c}
-p_{2} C_{3} \\
C_{4} / p_{2}
\end{array}\right) \\
& \left(\begin{array}{l}
A_{5} \\
A_{6}
\end{array}\right)=\left(\begin{array}{c}
C_{5} \\
C_{6}
\end{array}\right)
\end{aligned}
$$

Thus the solution is determined in the core near the core-film interface in terms of the $\mathrm{C}_{j}$.

An entirely analogous procedure can be applied in the liquid film. That is, asymptotic forms analogous to (29) can be joined to critical layer forms analogous to (38) near the interface $\left(r \rightarrow x_{*}, r \geqslant x_{*}\right)$ and near the wall $(r \rightarrow L, r \leqslant L)$. It turns out, however, that with the approximation to be made in Section 6 on exponential orders of magnitude, the same result is obtained when it is assumed that a critical 
layer form is valid throughout the film and the evaluations of $\zeta$ and $t$ are made locally:

$$
\begin{aligned}
& \hat{\zeta}=\left(\hat{\mu} \hat{t}^{2}\right)^{-1 / 3} \hat{A} \\
& \hat{t}=\frac{d \hat{A}}{d r}
\end{aligned}
$$

The $\hat{(})$ refers to evaluations in the film. Thus throughout the film, we take:

$$
\begin{aligned}
& v=\hat{A}_{1} \hat{Z}_{0}+\hat{A}_{2} \hat{\varphi}_{0}+\hat{A}_{5} v_{5}+\hat{A}_{6} v_{6} \\
& u=\hat{A}_{3} \hat{Z}_{2}+\hat{A}_{4} \hat{\varphi}_{2}+\hat{A}_{5} u_{5}+\hat{A}_{6} u_{6} \\
& w=\frac{1}{i \alpha_{4}}\left(\frac{\hat{t}}{\hat{\mu}}\right)^{1 / 3}\left(\hat{A}_{3} \hat{Z}_{1}+\hat{A}_{4} \hat{\Psi}_{1}\right) \\
& -\frac{m}{x_{\star} x_{*}}\left(\hat{A}_{1} \hat{Z}_{0}+\hat{A}_{2} \hat{\varphi}_{0}\right)+\hat{A}_{5} \omega_{5}+\hat{A}_{6} \omega_{6} \\
& \pi=\left(\hat{\varphi}^{2} \hat{t}\right)^{1 / 3}\left[\hat{A}_{3}\left(\hat{Z}_{4}+\hat{r} \hat{Z}_{3}-\hat{z}_{1}\right)+\hat{A}_{4}\left(\hat{\varphi}_{4}+\hat{\varphi} \hat{\varphi}_{3}-\hat{\varphi}\right)\right] \\
& +\hat{A}_{5} \pi_{5}+\hat{A}_{6} \pi_{6}
\end{aligned}
$$

where $\left(\hat{Z}_{k}, \hat{\varphi}_{k}\right) \equiv\left(Z_{k}(\hat{r}), \Psi_{k}(\hat{\zeta})\right)$.

6. The Eigenvalue Problem

The regularity conditions (6) have given three linear homogeneous conditions $(34,35)$ on the $C_{j}$. Applying the wall conditions (7) to the film solution (38) leads to three linear homogeneous conditions in. the $A_{j}$ :

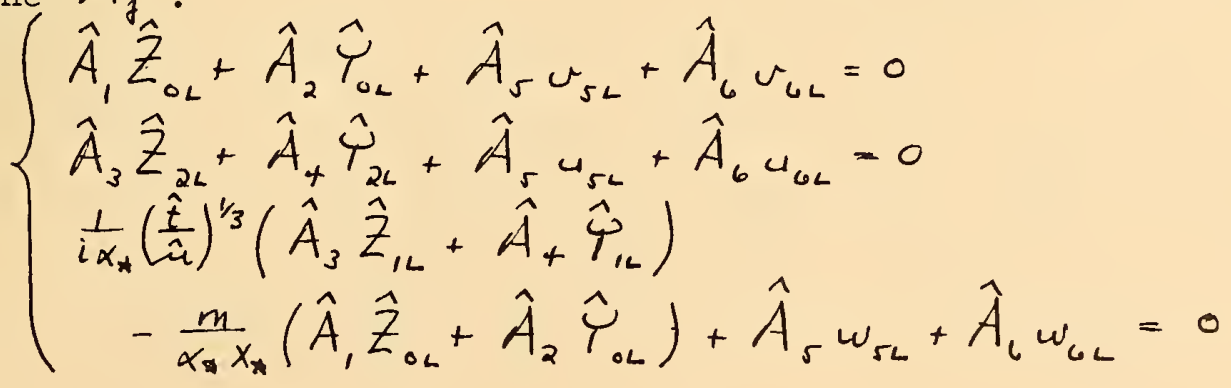

21 
where $\hat{Z}_{j L} \equiv Z_{j}(\hat{\varphi}(L))$.

The six interface conditions $(15,16)$ lead to six linear, homogeneous relations among the $\left(\hat{A}_{j}, C_{j}\right)$. We shall not write them down explicitly because of their length, but shall refer to them as (44) and (45) respectively:

$$
\left\{\begin{array}{l}
6 \text { linear homogeneous relations in }\left(\hat{A}_{j}, C_{j}\right) \\
\text { derived from interface conditions }(15,16)
\end{array}\right\} \text {. }
$$

The twelve linear homogeneous relations ( 34 or $35,43,44,45)$ suffice to determine nontrivial $\left(C_{j}, \hat{A}_{j}\right)$ to within a single scaling factor if the twelfth order determinant of the coefficient vanishes.

This determinant is given explicitly in Appendix 2 in the following form:

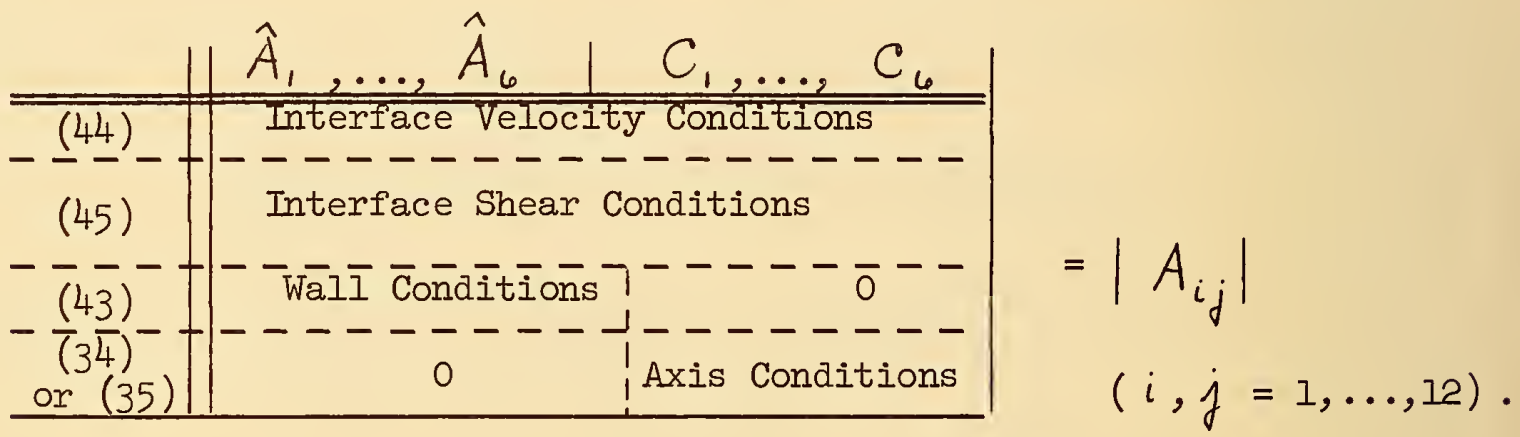

In the determinant, we have used:

$$
\begin{aligned}
& \hat{\mu}=m_{0} \mu \\
& \hat{p}=m_{1} \rho \\
& q_{\star}=i \alpha_{*}\left(m_{0}-1\right)+\frac{\sigma^{*}}{\mu x_{\star}\left(c_{*}-W_{x}\right)} \\
& \beta_{*}=\frac{+1}{i \alpha_{\star} \mu\left(W_{x}-c_{*}\right)}\left[\left(\tau_{0}^{\prime}\right)_{\text {liquid }}-\left(\tau_{0}^{\prime}\right)_{\text {gas }}\right]_{r=x_{*}}
\end{aligned}
$$

The prime reduction to be made in this determinant is the elimination of exponentially small terms, i.e., terms down $\theta\left(e^{-\mu^{-1 / 2} l}\right)$ for some $\ell$ from the leading terms. To do this, we first replace row 9, $R_{q}$, by (row 9); $R_{q}^{\prime}=R_{q}+\frac{m}{\alpha_{\star} x_{*}} R_{7}$. This makes $A_{q 1}=0, A_{q 2}=0$,

$$
A_{q 5}=w_{r_{L}}+\frac{m}{\alpha_{1} x_{1}} v_{5 L}, \quad A_{q 6}=w_{G L}+\frac{m}{\alpha_{1} x_{2}} v_{6 L} \text {, }
$$

22 
and does not effect the other elements. Now we divide columns 1 through 4 by, respectively, ( $\hat{Z}_{0 x}, \hat{\varphi}_{0 x}, \hat{Z}_{2 x}, \hat{\varphi}_{2 x}$ ) and columns 7 through 10 by, respectively, $\left(A_{17}, A_{18}, A_{29}, A_{210}\right)$, row 10 by $A_{18}$, row 12 by $A_{2,10}$. Then the first 6 rows are purely algebraic in $\mu$ (i.e., algebraic in $\zeta$ as $S \rightarrow \infty$ ). On the other hand (39):

$$
\frac{\hat{z}_{K L}}{\hat{z}_{n x}} \tilde{|\rho|} f_{K n}\left(\hat{r}_{L}, \hat{e}_{x}\right) e^{\frac{2}{3}\left(\hat{r}_{L}^{3 / 2}-\hat{r}_{x}^{3 / 2}\right)}
$$

for integral $(k, n)$, where $f_{k n}$ is algebraic in its arguments. Now the real part of $e^{3 / 2}$ :

$$
|r|^{3 / 2} \cos \left(\frac{3}{2} \operatorname{ang} r\right)=\left(\frac{\rho \alpha_{\pi} / W-c_{*} \mid}{\mu^{1 / 3}|t|^{2 / 3}}\right)^{3 / 2} \cdot\left\{\begin{array}{cc}
\cos \frac{q \pi}{4} & \left(r<r_{c}\right) \\
\cos \frac{3 \pi}{4} & \left(r>r_{c}\right)
\end{array}\right\}
$$

is monotonically decreasing with increasing $r$ in each medium. Hence

$$
\left|\frac{\hat{Z}_{K L}}{\hat{z}_{n x}}\right| \widetilde{\rho}\left|f_{K n}\right| e^{-\mu^{-1 / 2} \delta}
$$

for some positive $\delta$ independent of $\mu$ associated with $I-x$ and is exponentially small as $\mu \rightarrow 0$. On the other hand,

$$
\left|\frac{\hat{\varphi}_{K L}}{\hat{\varphi}_{n x}}\right| \underset{|F|}{\sim}\left|f_{k n}\right| e^{\mu^{1 / 2} \delta}
$$

is exponentially large as $\mu \rightarrow 0$. .

$$
\left|p_{0}\right|=\left|e^{-\mu^{-1 / 2} \int_{0}^{x_{1}} d r A^{1 / 2}}\right| \underset{\mu \rightarrow 0}{\sim} e^{\mu^{-1 / 2} X}
$$

is exponentially large as $\mu \rightarrow 0$, with $\underline{\bar{x}}$ related to the core radius $x$. Then

$$
\begin{aligned}
& \frac{P_{0}^{2} A_{18}}{A_{17}} \sim \frac{P_{0}^{2} A_{210}}{A_{29}} \sim e^{2 \mu^{-1 / 2} \underline{X}} \\
& \frac{P_{0}}{A_{17}} \sim \frac{P_{0}}{A_{29}} \sim e^{\mu^{-1 / 2} \underline{X}}
\end{aligned}
$$


Clearly the largest terms in the determinant will contain the factors:

$$
A_{107} \cdot A_{129} \cdot A_{1111} \cdot A_{72} \cdot\left(\begin{array}{l}
A_{84} \\
A_{94}
\end{array}\right)
$$

which is

$$
\approx e^{-1 / 2(2 \bar{X}+2 \delta)} .
$$

Then dropping the exponentially small terms, the vanishing of the $12 \times 12$ determinant leads to the vanishing of the $8 \times 8$ determinant obtained from the cofactor of

$$
A_{107} \cdot A_{129} \cdot A_{111} \cdot A_{72}
$$

in which we also set

$$
\begin{gathered}
\left(A_{83}, A_{93}\right)=0 \\
\left(A_{14}, A_{24}, \ldots, A_{64}\right)=0 .
\end{gathered}
$$

Now we replace row (3) by $\frac{m}{\alpha_{\star} x_{\star}}[$ row (1) + row (3) $]$, row (5) by $\left[\right.$ row $(5)+\frac{\alpha_{\star} x_{x}}{m}$ row (6) $-\left(\frac{m_{0}-1}{x_{\star}}\right)$ row (1)], and row (6) by $\left[\right.$ row $(6)-\frac{m}{\alpha_{\star} x_{\star}}$ row (5)], in that order, and use the row and column multipliers:

$\begin{array}{cccc}\text { row } & \text { mult } & \text { col } & \text { mult } \\ 1 & 1 & 1 & 1 \\ 2 & m & 3 & \frac{1}{m} \\ 3 & m & 4 & \frac{1}{m} \\ 4 & \frac{m}{x_{x} A_{x}}=\frac{m m_{1}}{x_{*} \hat{A}_{k}} & 5 & 1 \\ 5 & m^{2} x_{*} & 6 & 1 \\ 6 & \frac{x_{*}}{m} & 8 & 1 \\ 8 & m & 10 & \frac{1}{m} \\ 9 & m & 12 & 1\end{array}$

We obtain the $8 \times 8$ determinant as shown in Appendix 2.

One can now examine algebraic orders of magnitude in $\mu$ as $5 \rightarrow \infty$, and expand the determinant in the form

$$
\left(d_{e} t\right)=\left(d_{e} t\right)_{0} \mu^{-5 / 2}+\left(d_{e} t\right) \mu^{-2}+\theta\left(\mu^{-3 / 2}\right) \text {. }
$$


One obtains in this way, retaining the top two orders, a bilinear form

$$
\text { det }=\sum B_{i} \bar{B}_{i}
$$

of 11 terms, where the $B_{i}$ are the $3 \times 3$ minors of the "inviscid" terms, (cols. 5, 6, 12), while the $\bar{B}_{i}$ are dependent on the viscous elements, (cols. 1, 3, 4, 8, 10). The result is still sufficiently complicated to make further analysis fruitless and require numerical methods for further evaluation.

The $8 \times 8$ determinant will be put in a final dimensionless form by setting

$$
\begin{array}{ll}
\xi=r / L & \sum=\frac{\sigma *}{\hat{\rho} L\left(W_{x}\right)^{2}} \\
\alpha=\alpha_{*} L & \frac{1}{F^{2}}=\frac{g^{*} L}{\left(W_{x}\right)^{2}} \\
c=c_{\star} / W_{x} & \\
W(\xi)=W(r) / W_{x} & \\
x=x_{\star} / L & \hat{W_{x} L}
\end{array}
$$

so that Re is a Reynold's number, $Z$ the Froude number (dependent on sign $g^{*}$ ), and $\sum$ a dimensionless surface tension parameter. We divide column 5 by $v_{5 x}=-\frac{i m}{x_{x} \widehat{A}_{x}}$, column 6 by $u_{6 x}=\frac{1}{L \hat{A}_{x}}$, and column 12 by $\pi_{2 x}$. We have, then, finally:

$$
0=\Delta=\left|A_{i j}\right|
$$




$$
\Delta=\left|\begin{array}{cccccccc}
-1 & 0 & 0 & -1 & 0 & 1 & 0 & 1 \\
0 & -1 & 0 & 0 & A_{26} & 0 & 1 & A_{212} \\
0 & A_{33} & 0 & A_{35} & A_{36} & 0 & A_{310} & A_{312} \\
0 & A_{43} & 0 & A_{45} & A_{46} & 0 & A_{410} & A_{412} \\
0 & A_{53} & 0 & A_{55} & A_{56} & A_{58} & A_{510} & 0 \\
A_{61} & A_{63} & 0 & A_{65} & A_{66} & A_{68} & 0 & 0 \\
0 & 0 & 1 & A_{85} & A_{86} & 0 & 0 & 0 \\
0 & 0 & A_{94} & A_{95} & A_{96} & 0 & 0 & 0
\end{array}\right| .
$$

The values of the $A_{i j}$ follow.

(The validity of the basic reduction to an $8 \times 8$ determinant is difficult to assess. At some points of the critical curves computed, the exponential factors dropped were calculated to be down by only $e^{-2.5}$ from the factors retained, which is certainly not as much as one would wish. The effect of this on the location of the critical curves is, nevertheless, probably not very great, although it would be desirable to have some results for the complete $12 \times 12$ case.)

26 


$$
\begin{aligned}
& A_{61}=\frac{m_{0}}{\alpha} \frac{\hat{\mathscr{W}}_{x}^{\prime}}{1-c}\left(\hat{S}_{x} \frac{\hat{Z}_{-1 x}}{\hat{Z}_{0 x}}\right) \\
& A_{33}=-\frac{1}{i \alpha} \frac{\hat{F}_{x}^{\prime}}{1-c}\left(\hat{\rho}_{x} \frac{\hat{Z}_{1 x}}{\hat{Z}_{2 x}}\right)+\frac{W_{x}^{\prime}-\hat{W}_{x}^{\prime}}{i \alpha(1-c)} \\
& A_{43}=-\frac{m_{1} \hat{r}_{x}^{\prime}}{i \alpha x \operatorname{Re}(1-c)^{2}}\left(\hat{\rho}_{x} \frac{\hat{Z}_{1 x}+\hat{\rho}_{x} \hat{Z}_{3 x}+\hat{Z}_{4 x}}{\hat{Z}_{2 x}}\right) \\
& +\frac{m, \sum}{x(1-c)^{2}}\left(1+\frac{m^{2}-1}{\alpha^{2} x^{2}}\right) \\
& A_{53}=\alpha x\left[b+q\left(1+\frac{m^{2}}{\alpha^{2} x^{2}}\right)\right]+i m_{0} x^{2}\left(\frac{\hat{r}_{x}^{\prime}}{1-c}\right)^{2}\left(\frac{\hat{\rho}_{x}^{2} \hat{Z}_{0 x}}{\hat{z}_{2 x}}\right) \\
& A_{63}=-\frac{1}{\alpha^{2} x} q \\
& A_{94}=-\frac{1}{i \alpha}\left(\frac{\hat{r}_{1}^{\prime}}{c}\right)\left(\frac{\hat{\vec{r}}_{L} \hat{T}_{1 L}}{\hat{\varphi}_{2 L}}\right) \\
& A_{58}=-\left(m_{0}-1\right) m^{2} \\
& A_{68}=-\frac{1}{\alpha} \frac{W_{x}^{\prime}}{1-c}\left(\frac{\rho_{x} \varphi_{-1 x}}{\Psi_{0 x}}\right)+\frac{m_{0}-1}{\alpha x} \\
& A_{310}=\frac{1}{i \alpha} \frac{\Psi_{x}^{\prime}}{1-c}\left(\frac{P_{x} Y_{1 x}}{Y_{2 x}}\right)
\end{aligned}
$$

27 


$$
\begin{aligned}
& A_{410}=\frac{m_{1} r_{x}^{\prime}}{i \alpha m_{0} x \operatorname{ra}(1-c)^{2}}\left(\rho_{x} \frac{\varphi_{1 x}+\Gamma_{x} \varphi_{3 x}+\varphi_{+x}}{\varphi_{2 x}}\right) \\
& A_{510}=-i x^{2}\left(\frac{w_{x}{ }^{\prime}}{1-c}\right)^{2}\left(\frac{\Gamma_{x}^{2} \varphi_{0 x}}{Y_{2 x}}\right) \\
& A_{26}=i x \\
& A_{212}=-i H \\
& A_{35}=-\alpha x\left(1+\frac{m^{2}}{\alpha^{2} x^{2}}\right) \\
& A_{36}=-\frac{x W_{x}^{\prime}}{\alpha(1-c)} \\
& A_{312}=\frac{w_{x}^{\prime}}{\alpha(1-c)} H+\alpha \times\left(1+\frac{m^{2}}{\alpha^{2} x^{2}}\right) \\
& A_{45}=-i m_{1}-\frac{2 \alpha m_{1}}{R(1-c)}\left(1+\frac{m^{2}}{\alpha^{2} c^{2}}\right) \\
& A_{46}=-\frac{i m_{1} \sum}{(1-c)^{2}}\left(1+\frac{m^{2}-1}{\alpha^{2} x^{2}}\right)+\frac{2 m_{1}}{\alpha \operatorname{Ra}(1-c) x}\left(1-\frac{x \hat{\psi}_{x}^{\prime}}{1-c}\right) \\
& A_{+12}=i-\frac{2 m_{1} \alpha}{m_{0} r_{2}(1-c)}\left[\frac{H}{\alpha^{2} x^{2}}\left(1-\frac{x r_{x}^{\prime}}{1-c}\right)-\left(1+\frac{m^{2}}{\alpha^{2} x^{2}}\right)\right]
\end{aligned}
$$

28 


$$
\begin{aligned}
& A_{55}=-m_{0} m^{2} \\
& A_{56}=m_{0} \alpha^{2} x^{3}\left(1+\frac{m^{2}}{\alpha^{2} x^{2}}\right)-i \alpha x^{2}\left[-\sigma+q\left(1+\frac{m^{2}}{\alpha^{2} x^{2}}\right)\right] \\
& -m_{0} x^{2}\left(\frac{\hat{w}_{x}^{\prime}-x \hat{w}_{x}^{\prime \prime}}{1-c}\right) \\
& A_{5,2}=-m^{2}\left(m_{0}-2\right)-\alpha^{2} x^{2} H\left[\left(1+\frac{m^{2}}{\alpha^{2} x^{2}}\right)+\frac{x^{2} r_{x}^{\prime \prime}-x w_{x}^{\prime}}{\alpha^{2} x^{2}(1-c)}\right] \\
& A_{c s}=\frac{m_{0}}{\alpha}\left(\frac{1}{x}+\frac{{\hat{w_{x}^{\prime}}}_{x}}{1-c}\right) \\
& A_{66}=-x\left(\frac{m_{0}}{\alpha x}-\frac{i q}{\alpha^{2} x^{2}}\right) \\
& A_{612}=\frac{1}{\alpha x}\left(m_{0}-2-\frac{x w_{x}^{\prime}}{1-c}+H\right) \\
& A_{85}=i \times \frac{1-c}{c} Q_{51} \\
& A_{86}=i \times \frac{1-c}{c} Q_{6 L} \\
& A_{95}=-\frac{\alpha x(1-c)}{c}\left(1+\frac{m^{2}}{\alpha^{2} x}\right) \pi_{5 L}+\frac{x \psi_{1}^{\prime}(1-c)}{\alpha c^{2}} Q_{5 L}
\end{aligned}
$$

29 


$$
\begin{aligned}
& A_{q 6}=\frac{x(1-c) \hat{r}_{1}^{\prime}}{\alpha c^{2}} Q_{G L}-\frac{\alpha x(1-c)}{c}\left(1+\frac{m^{2}}{\alpha^{2} x^{2}}\right) \pi_{G L} \\
& q=i \alpha \times\left(m_{0}-1\right)-\frac{m_{0} \gamma \sum}{1-c} \\
& G=\frac{-i x}{\alpha(1-c)}\left(\frac{L^{2}}{u W_{x}}\right) /\left.\left(\frac{d}{d r} \tau_{0}(r)\right)_{r=x_{*}}\right|_{\text {gas }} ^{\text {loguid. }} \\
& =\frac{-i \times m_{0}}{\alpha(1-c)} \frac{m_{1}-1}{m_{1}} \frac{\gamma \varepsilon_{0}}{Z} \\
& \Gamma_{x}=z_{1} e^{i \arg r_{x}} \\
& z_{1}=11-c \mid\left(\frac{m_{0} \alpha \gamma}{m_{1}\left(r_{x}^{\prime}\right)^{2}}\right)^{1 / 3} \\
& \hat{e}_{x}=z_{2} e^{i \arg \hat{\rho}_{x}} \text {, } \\
& z_{2}=|1-c|\left(\frac{\alpha r_{2}}{\left(\hat{r}_{x}^{\prime}\right)^{2}}\right)^{1 / 3} \\
& \hat{\zeta}_{L}=z_{3} e^{i \arg \hat{\rho}_{L}} \text {, } \\
& z_{3}=|c|\left(\frac{\alpha r_{e}}{\left(\hat{r}_{1}\right)^{2}}\right)^{1 / 3}
\end{aligned}
$$

30 


\section{Base Flow}

The inviscid disturbance solutions depend on the entire base flow velocity profile $\mathcal{W}(\xi)$, while the viscous solutions depend on certain local properties of $W$. Strictly speaking a stability analysis has meaning only when applied to an exact steady solution of the Navier-Stokes equations. (It has been applied with success, however, to boundary layer flow when the streamlines are assumed to be parallel to the wall.) The velocity profile for laminar pipe flow is exactly a function of the radius only. Feldman's analysis, however, shows instability of a two-phase flow to disturbances of wave length associated with film thickness while instability in homogeneous Poiseuille flow occurs only for wave lengths comparable to the radius. Then to some extent, we may be able to distinguish in the thin film flow two types of instability, the one associated with the gas core, the other with the film-core interface. It may be possible that the core be turbulent while in a neighborhood of the thin film the turbulent fluctuations could be of so small a magnitude as to be contained in a linearized stability theory. Near the film in this case the turbulent core makes itself felt through a radical change in mean velocity profile and shear stress. Then in a non-rigorous way one might extend the stability analysis to thin film flows with velocity profiles of a turbulent type. This extension is not included in this note.

We shall adopt velocity profiles in the gas core and liquid film as follows:

$$
\begin{aligned}
& \mathscr{W}_{g}(\xi)=k-f s^{2} \\
& W_{l}(s)=-W_{1}^{\prime}(1-\xi)+\lambda(1-s)^{2} \\
& w_{g}(x)=W_{l}(x)=1 .
\end{aligned}
$$

The core flow profile is of the correct form for.laminar flow. A determination of the constants $\left(K, f, W_{1}^{\prime}, \lambda\right)$ must be made. The requirements $\frac{d p}{d z}=-r=$ constant, $W=W(r)$ only, lead to the base flow equation

$$
-u\left(W^{\prime \prime}+\frac{1}{r} W^{\prime}\right)+\left(r-\rho g^{\prime}\right)=0,
$$


which has the general solution in non-dimensional form

$$
\begin{gathered}
W(\xi)=A_{0} \ln \xi+A_{1}-\Gamma \xi^{2} \\
\Gamma \equiv \frac{r-\rho g^{+}}{4 \mu} \frac{L^{2}}{W_{x}^{2}} .
\end{gathered}
$$

In the gas core then

$$
\begin{aligned}
W_{g}(\rho)=B_{1}-\Gamma_{g} \xi^{2} \\
\Gamma_{g}=\frac{\gamma-\rho g}{4 \mu} \frac{L^{2}}{W_{\lambda}^{2}}
\end{aligned}
$$

while in the liquid film, to 2nd order in film thickness:

$$
\begin{aligned}
W_{l}(\xi)= & A_{0} \ln (1-(1-\xi))+A_{1} \\
& -\Gamma_{l}(1-(1-\rho))^{2} \\
\doteq & A_{0}\left[(1-\rho)+\frac{1}{2}(1-\rho)^{2}\right] \\
& +\Gamma_{l}\left[2(1-\xi)-(1-\rho)^{2}\right] \\
\Gamma_{l} \equiv & \frac{\gamma-\hat{\rho} g}{4 \hat{\mu}} \frac{L^{2}}{W_{x}^{2}}
\end{aligned}
$$

We have the wall condition

$$
W_{e}(1)=0
$$

the interface velocity conditions

$$
W_{l}(x)=q_{g}(x)=1
$$

and the interface shear condition

$$
m_{0} w_{l}^{\prime}(x)=w_{g}^{\prime}(x)
$$


to determine the unknown constants. Identifying the resulting forms with equations 49 through 51, we find:

(59)

$$
\begin{aligned}
& n_{g}=k-f \xi^{2} \\
& w_{l}=-w_{1}^{\prime}(1-p)+\lambda(1-\beta)^{2} \\
& x=1+f x^{2} \\
& f=\frac{m_{0}}{1-x^{2}}+\frac{m_{0}\left(m_{1}-1\right)}{2 m_{1}} \frac{\gamma_{e}}{z^{2}} \frac{1-x}{(1+x)(2-x)} \\
& W_{1}^{\prime}=-\frac{2}{1-x^{2}}+\frac{m_{1}-1}{m_{1}} \frac{\gamma_{2}}{t^{2}} \frac{x(1-x)}{(1+x)(2-x)} \\
& \left\{\lambda=-\frac{1}{1-x^{2}}+\frac{m_{1}-1}{m_{1}} \frac{R_{1}}{t^{2}} \frac{x}{(1+x)(2-x)}\right. \\
& r=\frac{W_{x}^{2} \hat{\rho}}{L}\left[\frac{4}{R_{e}\left(1-x^{2}\right)}\right. \\
& \left.+\frac{1}{t^{2}}\left(1-\frac{m_{1}-1}{m_{1}} \frac{x(3-x)}{(1+x)(2-x)}\right)\right] \\
& W_{x}^{\prime}=-2 f x \\
& \hat{W}_{x}^{\prime}=w_{1}^{\prime}-2 \lambda(1-x)
\end{aligned}
$$




\section{Numerical Computation}

It did not appear that anything much could be done analytically with this $8 \times 8$ complex determinant, even in the thin film case with linear velocity profiles, corresponding to Feldman's study. He, indeed, used numerical methods also.

The $\mu=0$ limit, the so-called "inviscid limit," may be obtained from the determinant, and corresponds to setting (See Appendix 6):

$$
\Delta_{\mu=0}=\left|\begin{array}{ccc}
0 & A_{26} & A_{212} \\
A_{45} & A_{+6} & A_{412} \\
A_{85} & A_{86} & 0
\end{array}\right|=0
$$

The same result is readily found by solving the inviscid boundary value problem from scratch. (Feldman goes to same length to excuse the fact that his two inviscid limits differ, when in fact they do not, as a simple algebraic manipulation shows!) While the inviscid solution cannot give a "critical Reynolds number" for stability, it locates any non-stable domains in the $(\alpha, \bigvee)$ plane which extend to infinity in $\gamma e$, and provides the inviscid limit of one of the two functions defined by the vanishing of the $8 \times 8$ complex determinant $\triangle$;

$$
\Delta=E\left(\alpha, R_{e}, c\right)+i F\left(\alpha, \gamma_{e}, c\right)=0
$$

Searching this limit curve, $F(\alpha, \infty, c)=0$ in the approach used, enabled us to find a zero $\Delta(\alpha, \gamma e, c)=0$ quite easily, even though the resulting critical Reynolds number ( $\left(\mathrm{C}_{\mathrm{C}}\right)_{\text {a }}$ was not very large. Varying the neutral wave speed $c$ by small steps, one is able to trace out a neutral stability curve:

$$
\begin{aligned}
& \left\{\begin{array}{l}
E(\alpha, \gamma, c)=0 \\
F(\alpha, \gamma, c)=0
\end{array}\right. \\
& \left\{\begin{array}{l}
\alpha=\alpha(c) \\
\gamma e=\gamma(c)
\end{array}\right.
\end{aligned}
$$


defined by the parameter $c$. These curves have a nose defining a minimum ( $\gamma_{e_{a}}, \alpha_{a}$ ) for which the flow is unstable. The position of this nose, the critical $\gamma \mathrm{C}_{\mathrm{C}}=\mathrm{C}_{\boldsymbol{a}}$, is then sought for other flows by small variation of the other parameters:

$$
\left\{\begin{array}{l}
h=1-x \\
m \\
m_{0} \\
m_{1} \\
\frac{t^{2}}{\sum}
\end{array}\right.
$$

All this would require a great deal of computing, and attempts to simplify $\triangle$ further were made.

First, all the elements $A_{i j}$ were expanded in the top two orders in $\gamma$, and then $\Delta$ itself was expanded, retaining only the top two orders again. It was found that the order of magnitude of $\gamma_{\mu_{4}}, \theta$ (100), was far too small to justify the asymptotic expansion used, values of 5 of $\theta\left(10^{-1}\right)$ occurring in some cases, where $P=\theta$ (10) would be required to use asymptotic forms for the $\Psi_{i}(\rho), Z_{i}(\zeta)$. Since full range numerical tabulations of $\psi$ and $Z^{[6]}$ are sparse in $\zeta$, and with too few significant figures in the mid-range $2 \leq|\varphi| \leq 8$, and since complex routines for $a_{i}(S)$, Bi $(S)$ had recently been written and checked out $[7]$, further routines were written (see Appendix 4) to permit at least four-place evaluation of the required functions on $0 \leq|e|<\infty$. Also, the entire determinant is calculated using single-precision complex arithmetic with no restriction on the order of $\boldsymbol{k}_{\mathrm{a}}$.

A more satisfactory simplification was the approximations made for $H$ and for $\left(\pi_{5 L}, Q_{5 L}, \pi_{G L}, Q_{G L}\right)$. When the inviscid equations for $\Pi(\xi)$ and $H(\bar{\sigma})$ are solved numerically, typical times for these alone were a minute. The approximating forms used (see Appendix 
E) are very good for thin films $h \leqslant 0 . /$, and probably adequate to $h \approx 0.5$. Numerical checks have shown that $\mathbb{R}_{c}$ is not significantly affected by using the approximate forms for thin films, but no comparisons were made at the thicker films values because of the long computing times required. Our primary interest is in the thin film anyway, as being the normal form in annular flow.

In its final form, in evaluation of the elements $A_{i j}$ and $\triangle$ took about 0.003 minutes. A single $\boldsymbol{R}_{\text {u }}(h)$ curve for $(0.001 \leq h \leq 0.3)$ took 30 minutes at a minimum.

In actual fact, the variations of the nose of the critical curve with respect to the parameters computed were:

$$
\frac{\partial R_{F}}{\partial h}, \frac{\partial R_{F}}{\partial m_{0}}, \frac{\partial R_{F}}{\partial m_{1}}, \frac{\partial R_{F}}{\partial \sigma}, \frac{\partial R_{F}}{\partial g}, \frac{\partial R_{F}}{\partial \beta}
$$

where

$$
\begin{aligned}
& g \equiv \frac{\gamma \varepsilon_{F}}{h{ }^{\prime}} \operatorname{sgn} g^{*}=\frac{\hat{\rho}}{\hat{\mu}} \sqrt{\left|g^{*}\right| L^{3}} \operatorname{sgn} g^{*} \\
& \sigma \equiv \frac{\sum Q_{F}}{h^{2}}=\frac{\sigma^{*} L \hat{\rho}}{\hat{\mu}^{2}} \\
& \beta \equiv \frac{m \alpha}{x}
\end{aligned}
$$

defining, respectively, gravity and surface tension parameters independent of flow rates, and an interface azimuthal wave number factor, or "wave pitch". Also, the derivative:

$$
\frac{\partial c_{I}}{\partial R_{F}}
$$

was computed on the nose curve to measure the rate of growth of the disturbance moving off the neutral line in the direction of increasing $R_{F}$. It is found from the requirement:

$$
\delta \Delta=\frac{\partial \Delta}{\partial R_{F}} \delta R_{F}+\frac{\partial \Delta}{\partial c} \delta c=0
$$


where

$$
\delta c=\delta c_{R}+i \delta c_{I}
$$

and $c_{I}=0$ on neutral curves. This complex equation leads to:

$$
\frac{\partial c_{I}}{\partial R_{F}}=\frac{\frac{\partial E}{\partial R_{F}} \frac{\partial F}{\partial c}-\frac{\partial F}{\partial R_{F}} \frac{\partial E}{\partial c}}{\left(\frac{\partial F}{\partial c}\right)^{2}+\left(\frac{\partial E}{\partial c}\right)^{2}}
$$

the partial derivatives being estimated by taking small independent changes in $W_{F}$ and real $c$. We must expect $\frac{\partial c_{I}}{\partial C_{F}}>0$ if the flow is

$$
\frac{\partial c_{I}}{\partial R_{F}}>0
$$

unstable for values of $\widehat{C}_{F}$ exceeding the values on the neutral curve.

Because of the extensive computing required, most computing was done for the air-water case, taking

$$
\begin{aligned}
& m_{0}=47.0 \\
& m_{1}=814.4
\end{aligned}
$$

The automated computation process, curve following by using a linear-variation predictor-corrector method, broke down for thick films, and could not be continued. In the cases shown, all values appeared numerically sound; values showing instability have not been included.

To be more precise, stariing from some known point on an $\left(\alpha_{F}, \gamma_{F}\right)$ curve, $\alpha_{F}$ was stepped by a small increment, then $\left(\gamma_{F}, c\right)$ readjusted to lie on curve, using the complex equation

$$
\delta \Delta=0=\frac{\partial \Delta}{\partial \alpha_{F}} \delta \alpha_{F}+\frac{\partial \Delta}{\partial R_{F}} \delta R_{F}+\frac{\partial \Delta}{\partial c} \delta c
$$

to determine $\left(\delta c, \delta \ell_{F}\right)$, and small variations in $\left(\alpha_{F}, c, \Omega_{F}\right)$ to compute the partial derivatives. We continue to follow this curve through decreasing values of $\mathcal{C}_{F}$ until $R_{F}$ increases, and call the smallest value $\left(\gamma_{F}\right)_{C} \cdot$ In a similar way, starting from a point on one $\left(\alpha_{F}, \zeta_{F}\right)$ curve, one finds a point on another curve $\left(\alpha_{F}, \gamma_{F}\right)$ 
corresponding to changing some parameter $p$ into $p+\delta p$ by solving

$$
\delta \Delta=0=\frac{\partial \Delta}{\partial p} \delta p+\frac{\partial \Delta}{\partial c} \delta c+\frac{\partial \Delta}{\partial \gamma_{F}} \delta R_{F}
$$

and this curve is again followed to a nose $\left(\left(\ell_{C_{F}}\right)_{c s},\left(\alpha_{F}\right)_{c}\right)$. Breakdown of this procedure appears to occur when the estimates of partial derivatives fail to give useful predictions. While one would like to take smaller variation tken to compute partial derivatives, one is then limited by the four to five place accuracy of the computations.

$\partial R_{F}$ At each point near the nose of an $\left(\alpha_{F}, \gamma \zeta_{F}\right)$ - curve, variations $\frac{\partial R_{F}}{\partial p}$ in the position of the critical curve for fixed $\alpha_{F}$ are made for parameters $h, m_{0}, m_{1}, \sigma, g, \beta$ as an estimate of the change to be expected $\frac{\left(\delta \mathrm{C}_{F}\right)_{\boldsymbol{r}}}{\delta \rho}$. Much of the irregularity of these derivatives is doubtless due to the irregularity of the determination of $\left(\gamma_{F}\right)_{\boldsymbol{c}}$, due to the discrete stepping of $\alpha_{F}$.

9. Results

Because of the large number of parameters involved in this stability analysis and the large computing times involved, it has not been practical to generate neutral stability curves $\left(\alpha_{F}, \gamma_{F}\right)$ and critical curves $\left(\left(\gamma e_{F}\right)_{e}, h\right)$ for the wide variety of materials and flow conditions common in moderm engineering. For this reason, the results given are all for fluids with density ratio $m_{1}=814.4$ and viscosity ratio $m_{0}=47.0$, reasonably good values for an air-water system. The derivatives $\left(\frac{\partial R_{F}}{\partial m_{0}}, \frac{\partial R_{F}}{\partial m_{1}}\right)$ can be used to estimate the critical curve changes for fluids with somewhat different ratios $m_{0}$ and $m_{1}$.

Tables I through VII contain the computed data on the critical curves as functions of the basic parameters $\sigma, h, \beta, g$. The values of $\left(\gamma_{F}\right)_{c}, c$ and $\left(\alpha_{F}\right)_{c u}$ given are those nearest the nose of the particular neutral stability curve $\left(\alpha_{F}, \Omega_{F}\right)$ obtained by stepping through discrete increments, a change of from one to five percent as seemed desirable. This discretization in $\alpha_{F}$ is the major cause of the irregularity in the differences in derivatives shown with respect to $h$, as was remarked earlier. Data are plotted in figures $1-9$. 
The tables are arranged in order of increasing surface tension parameter, $0 \leq \sigma \leq .23(10)^{7}$. Within each table, the non-gravity flows are given first, all variables given as functions of film thickness $h$ for increasing values of wave pitch $\beta$. In the last two tables, Tables VI and VII , for which a gravity flow is given in each case, the $\beta=0$ curve develops complexities at larger values at $h$ which are difficult to trace with assurance because of the rapid changes. There is most certainly more than one curve involved, and consequent intersections evidenced by changes in sign of $\frac{\partial c_{I}}{\partial r_{F}}$; the linear extrapolation method cannot cope with these complications. For values of $h$ smaller than those at which these problems arise, however, there is a change in the sign of $\frac{\partial \gamma_{F}}{\partial \beta}$, indicating that the axially symmetric wave is not the least stable for larger values of $h$. The scattered values of $\beta$ included beyond this indicate the locus of $\frac{\partial \mathbb{Q}_{F}}{\partial \beta}=0$, and should give then the lowest critical Reynolds number ( $\left.G_{f}\right)_{c}$ in its dependence on both $h$ and $\beta$. Trends are not, in general, easily characterized, and the following generalizing remarks should be checked in individual cases:

(1) In the absence of gravity, surface tension is destabilizing for very thin films, stabilizing for thick films*. For gravity flows, a second destabilizing trend occurs at the thickest films studied. *( For the very thick films, $h \geqslant .15$, an unaccounted for irregularity in $\frac{\partial R_{F}}{\partial \sigma}$ with $h$ occurs.)

(2) For very thin films, $\frac{\partial R_{F}}{\partial m_{0}}$ is positive while $\frac{\partial R_{F}}{\partial m_{1}}$ is negative. These signs change at approximately the same small value of $h$, so that for all larger $h$, increased viscosity ratio is destabilizing, increased density ratio is stabilizing.

(3) For very thin films, increasing the downward (-g) acceleration due to gravity destabilized the flow, while for non-thin films it stabilizes the flow. (Again, an unexplained irregularity in $\frac{\partial R_{F}}{\partial \sigma}$ appears for very thick films.)

(4) Thin films are more stable for $\beta>0$ than for $\beta=0$, while for nonthin films, there are many cases in which the reverse is true. 
Figure 10 is extracted from Charvonia's paper, and shows a disturbance instability line based on apparent droplet entrainment occurring in experimental air-water f'lows downward in vertical pipes. Charvonia's dimensionless parameters are thickness parameter $T_{p}$ and liquid Reynolds number $N_{\mathbb{R}_{1}}$ :

$$
\begin{aligned}
& T_{p}=h^{*}\left[\frac{1}{g^{*}}\left(\frac{\hat{p}}{\hat{i}}\right)^{2}\right]^{1 / 3} \\
& N_{R_{1}}=\frac{4 G^{\prime}}{g^{*} \hat{\imath}}
\end{aligned}
$$

where $G^{\prime}$ is the peripheral liquid flow rate:

$$
G^{\prime}=\frac{1}{2} h^{*} \hat{\rho} W_{x}
$$

for thin films. Using his pipe radius $L=1.25^{\prime \prime}$ and $\hat{\mu}=2.0910^{-5}\left(\frac{\text { s/ugs }}{\text { ft-sec }}\right)$

$$
\begin{aligned}
& T_{P}=678 . \mathrm{h} \\
& N_{R_{1}}=2 R_{F} .
\end{aligned}
$$

The critical curves of figures 8 and 9 are plotted as curves 8 and 9 on rigure 10. Charvonia's experiments lead to:

$$
\begin{aligned}
& g=\frac{\left(g^{*} L^{3}\right)^{1 / 2} \hat{\rho}}{\hat{\mu}} \doteq 0.360(10)^{5} \\
& \sigma=\frac{\sigma^{*} L \hat{\rho}}{\hat{\mu}^{2}} \doteq 0.230(10)^{7}
\end{aligned}
$$

corresponding to curve 9. Curve 8 represents intermediate values for and $\sigma$.

\section{ACKNOWLEDGEMMENT:}

The author wishes to thank Miss Garney Hardy and Mr. M. D. Bunch for their strenuous efforts in programming portions of the numerical scheme. 


\section{References}

[1] C. C. Lin, "Theory of Hydrodynamic Stability," Cambridge University Press, (1955).

[2] S. Feldman, "On the Hydrodynamic Stability of Two Viscous Incompressible Fluids in Parallel Uniform Shearing Motion," J. Fluid Mech. ㄹ, 343, (1957).

[3] T. Brooke Benjamin, "Three-Dimensional Disturbances on an Unstable Film," J. Fluid Mech. 10, 3, 401, (1961).

[4] D. A. Charvonia, "A Study of the Mean Thickness of the Liquid Film and the Characteristics of the Interfacial Surface in Annular, TwoPhase Flow in a Vertical Pipe," Purdue University, Lafayette, Indiana, (May 1959).

[5] H. Schlicting, "Boundary Layer Theory," McGraw-Hill, (1960).

[6] H. Holstein, "Uber die Aussere und Innere Reibungsschicht bei Storungen Laminarer Stromungen," Z. Angwe. Math. Mech. 30, 25, (1950).

[7] 0. N. Strand, Private Communication, (1964).

[8] H. B. Squire, "On the Stability of Three-Dimensional Distribution of Viscous Fluid between Parallel Walls," Proc. Roy. Soc. Iondon, A, 142, (1933). 


\section{Glossary}

Dimensions are indicated in brackets: [L] length, [T] time, [M] mass, and combinations of them.

Subscripts $\mathrm{x}$ and $\mathrm{L}$ indicate evaluations at $\xi=\mathrm{x}$ and $\xi=\mathrm{L}$, respectively. Cap, $(\hat{)}$, implies a liquid film quantity.

$A\left(r^{*}\right)=i \alpha_{*} p\left(W-c_{*}\right)$ : coefficient in linearized equations $(2-4) \cdot\left[\mathrm{ML}^{-3} \mathrm{~T}\right]$

$A_{i j}$ : elements of the stability determinant, $\triangle$.

$b=\frac{i x m_{0}}{\alpha c} \frac{m_{1}-1}{m_{1}} \frac{R}{\tau^{2}} \quad:$ quantity in $\Delta$, see p. 30. [I]

$c=c_{R}+i c_{I}$ : complex wave speed; $c_{I}=0$ on neutral curve. [I]

$c_{*} \doteq \mathrm{W}_{\mathrm{X}} c \cdot\left[\mathrm{IT}^{-1}\right]$

E: real $(\triangle)$

$\mathrm{F}$ : imaginary $(\triangle)$

Z: Froude number, $\frac{W_{x} \text { squ } g^{*}}{\sqrt{1 g^{*} / L}}$.

$g=\frac{\hat{p}}{\dot{k}} \sqrt{\mid g^{*} / L^{3}} \operatorname{sqn} g^{*}$
rates. [I]

$g^{*}$ : gravitational constant. $\left[\mathrm{IT}^{-2}\right]$

$h$ : film thickness, $1-x^{*} / I$. [I]

H: parameter based on inviscid disturbances in the gas core. [1]

$i_{m, n}:$ numbers defined by asymptotic expansions of viscous flow near $r=0$ (see Appendix 3).

L: pipe radius. [L]

$m$ : azimuthal mode number in wave factor $e^{i m \varphi}$.

$m_{0}$ : viscosity ratio, $\hat{\mu} / \mu$. [I]

$m_{1}$ : density ratio, $\hat{\rho} / p$. [I]

$P_{0}, P_{2}$ : exponential factors occurring in viscous flow in core (see pp. $13-14)$.

$q=i \alpha x\left(m_{0}-1\right)-\frac{m_{0} R \Sigma}{1-c}:$ quantity in $A_{i j}$, see p. 30. [I] 


$$
\begin{aligned}
& q_{*}=q / x^{*} .[\mathrm{I}] \\
& Q(\xi)=\pi^{\prime}(\xi) .
\end{aligned}
$$

$r$ : radial coordinate. [L]

$\gamma_{e}=\frac{\hat{\rho} W_{x} L}{\hat{\mu}}$, pipe Reynolas number. [I]

$\gamma_{e_{F}}=h$ Re, film Reynolds number. [I]

$\left(\gamma_{F_{F}}\right)_{a}$ : critical film Reynolds number, a minimum with respect to $\alpha$, dependent on other parameters. [1]

$t^{*}$ : time coordinate [T]

$u(r), v(r), \omega(r)$ : radial, azimuthal and axial velocity coefficients for disturbance wave factor $e^{i \alpha_{*}\left(z^{*}-c_{\star} t^{*}\right)+i m \varphi} \quad .\left[\mathrm{IT}^{-1}\right]$

$W(r)$ : undisturbed axial velocity profile. $\left[\mathrm{LT}^{-1}\right]$

$\mathscr{W}(\xi)=\mathrm{W} / \mathrm{w}_{\mathrm{x}}$.

$\mathrm{x}: \mathrm{x}_{*} / \mathrm{L}$. [I]

$\mathrm{x}_{*}$ : undisturbed interface radius. [L]

$Y(\zeta), Z(\zeta)$ : functions describing flow near viscous critical layer $W=c$; see $(37)$.

$z^{*}$ : axial coordinate. [L]

$\alpha: \alpha_{*}^{\mathrm{I}}$.

$\alpha_{*}$ : wave number for axial component of wave factor $e^{i \alpha_{*} z^{*}}$. [I $\left.\mathrm{I}^{-1}\right]$

$\beta=\frac{m}{\alpha x}$; interface azimuthal wave number factor, or "wave pitch". [1]

$\beta_{*}=b / x_{*}$.

$\gamma$ : axial base flow pressure gradient: $\left[\mathrm{ML}^{-2} \mathrm{~T}^{-2}\right.$ ]

$\gamma^{*}, \gamma^{* *}$ : numbers defined by asymptotic expansions of viscous flow near $r=0$ (see Appendix 3).

$\Delta=E+i F$ : complex determinant whose vanishing defines neutral stability curves.

$\zeta$ : coordinate basic to viscous flow near critical layer $W=c$. 
$\mu:$ dynamic viscosity. $\left[\mathrm{ML}^{-I_{\mathrm{T}}}{ }^{-1}\right]$

$\rho=r / L \cdot[1]$

$\pi(r):$ pressure coefficient for disturbance wave factor

$$
e^{i \alpha_{*}\left(z^{*}-c_{*} t^{*}\right)+i m \varphi} \cdot\left[\mathrm{ML}^{-I_{\mathrm{T}^{-2}}}\right]
$$

$\Pi(\xi)$ : inviscid pressure coefficient.

$\Pi_{2}(\xi)$ : pressure coefficient satisfying $\Pi_{2}(\xi) \rightarrow \xi^{m}$ as $\xi \rightarrow 0$.

$\pi_{5}(\xi)$ : pressure coefficient satisfying

$$
\left(\pi T_{5}(x), Q_{5}(x)\right)=(1,0) \text {. }
$$

$\pi_{6}\left(\xi^{\circ}\right)$ : pressure coefficient satisfying

$$
\left(\pi_{6}(x), \quad Q_{6}(x)\right)=(0,1) \text {. }
$$

$\rho:$ density. $\left[\mathrm{ML}^{-3}\right]$

$\sigma=\frac{\sigma^{*} L \hat{p}}{\hat{\mu}^{2}}$ : a surface tension number independent of flow rates. [1] $\sigma *=$ surface tension. $\left[\mathrm{MT}^{-2}\right]$

$\Sigma=\frac{\sigma^{*}}{\hat{\rho} L\left(W_{x}\right)^{2}} \quad$ : Weber's number. [1]

$\sigma_{r r}{ }^{0}(r): \overline{F r}$-component of base flow stress tensor. $\left[\mathrm{ML}^{-I_{T}}{ }^{-2}\right]$

$\tau_{0}(r): \widetilde{r z}$-component of base flow stress tensor. $\left[\mathrm{ML}^{-1} \mathrm{~T}^{-2}\right]$

$\varphi$ : azimuthal coordinate, in radians. 
Appendix 1

$12 \times 12$ DETERMINANTT

Elements of the basic $12 \times 12$ determinant are given in the following charts. 


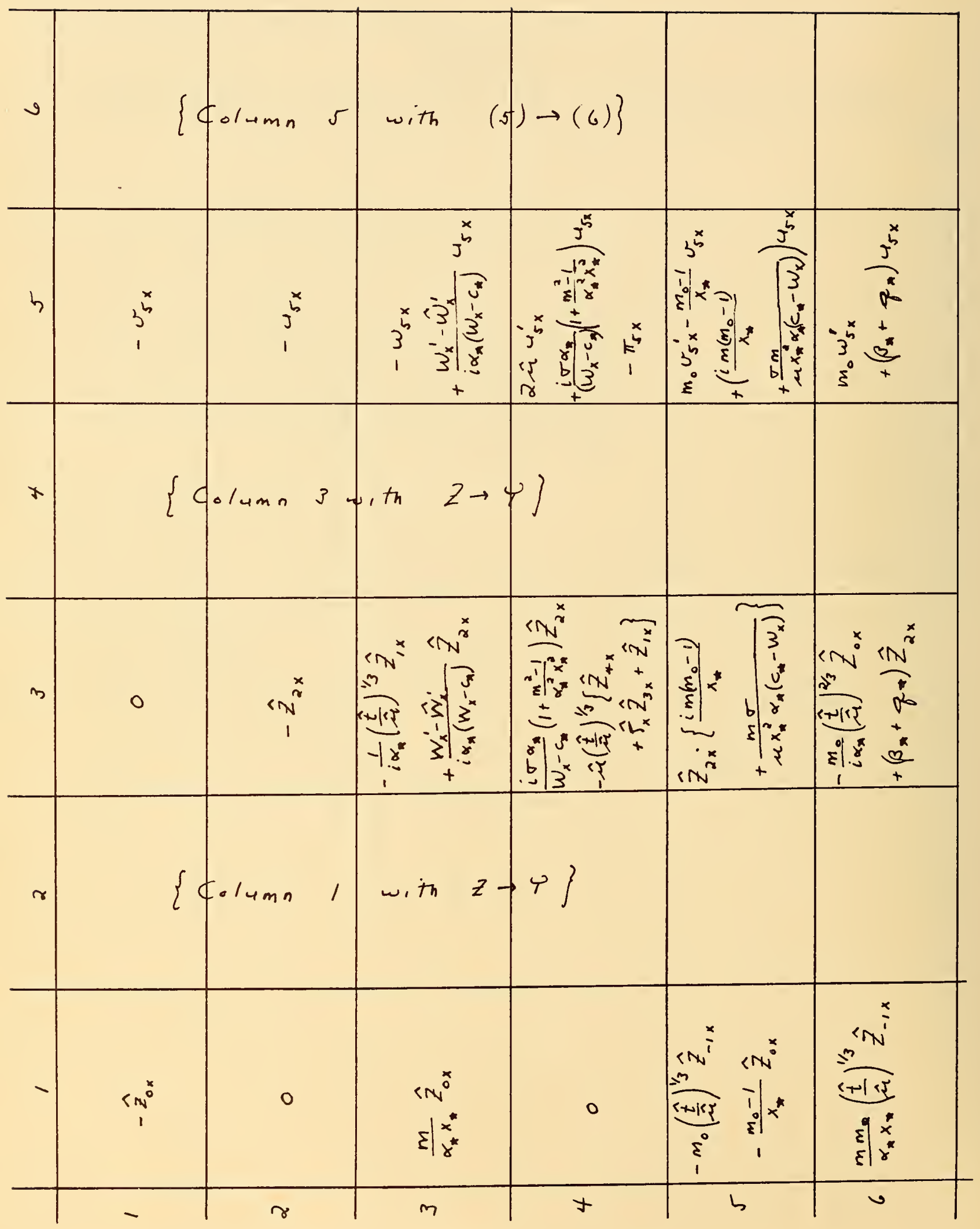




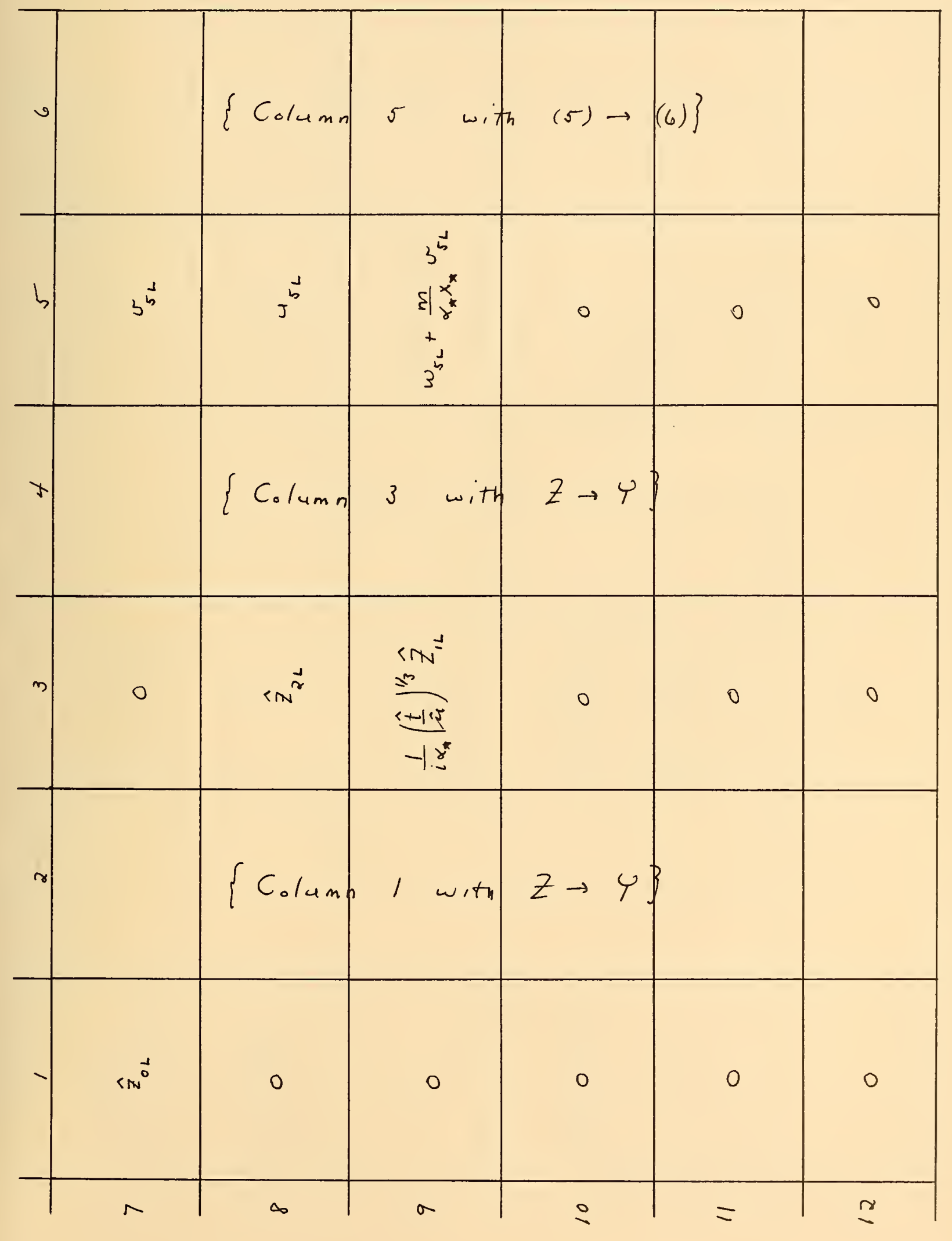




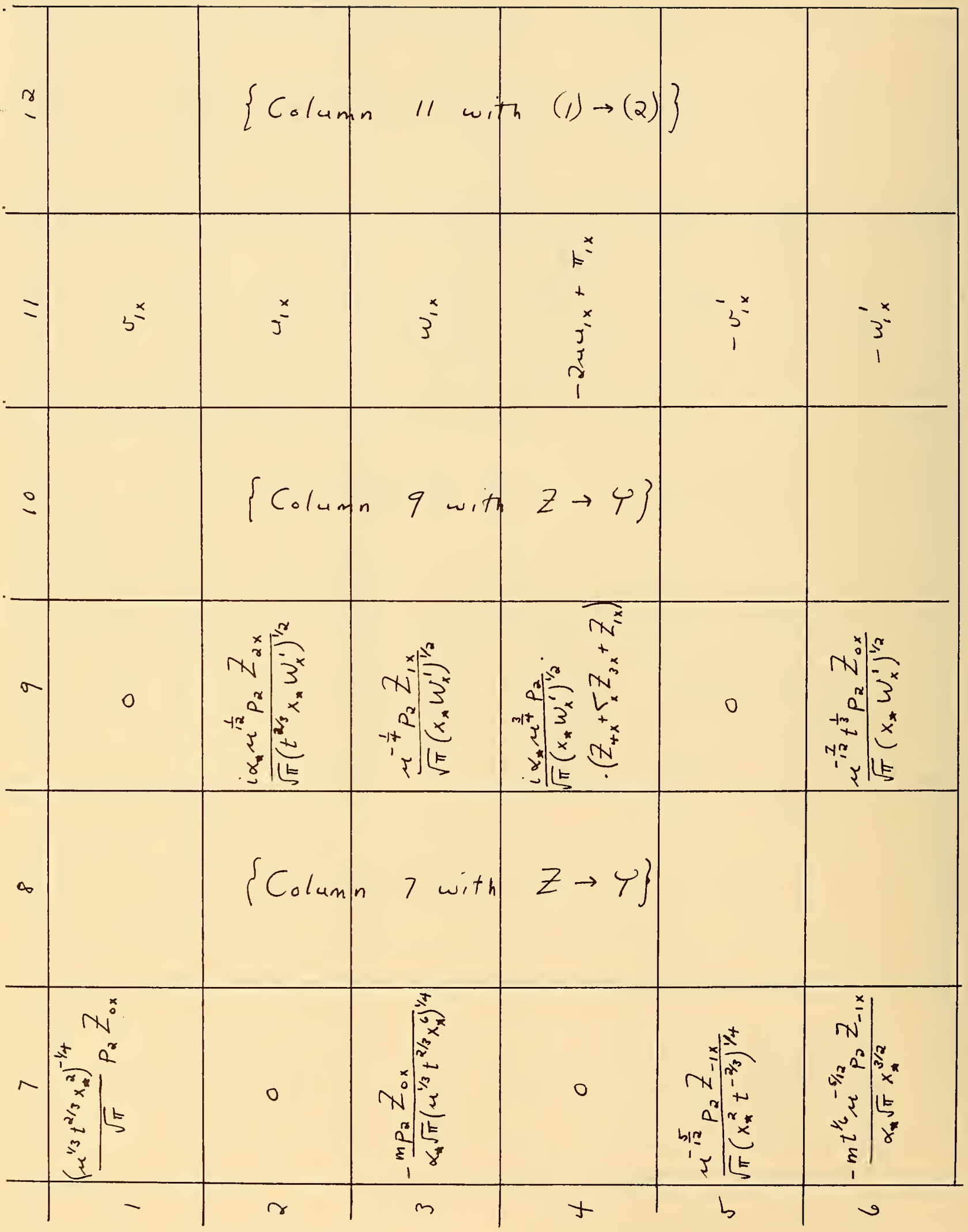




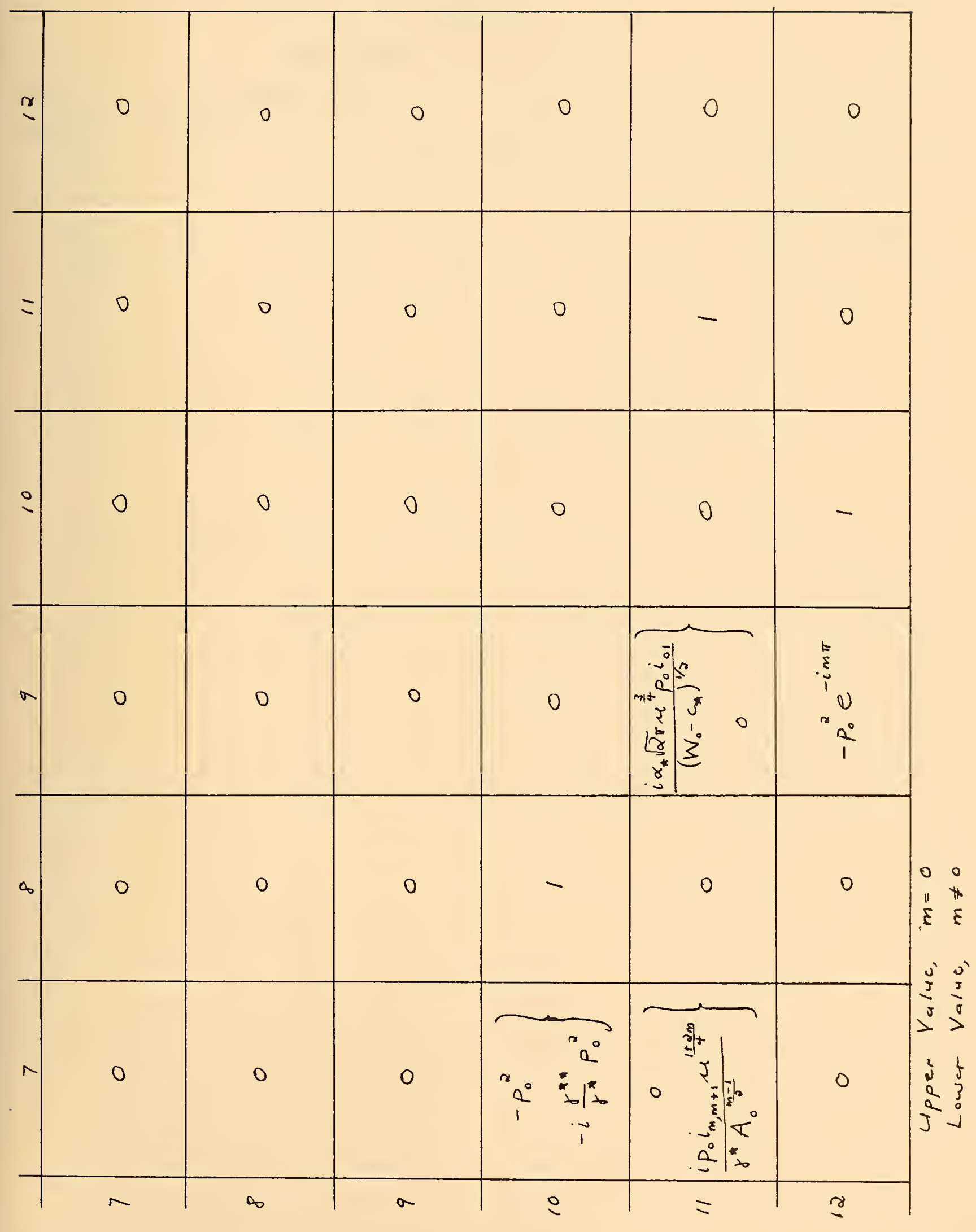


Appendix 2

INTERMEDIATE $8 \times 8$ DETERMTINANT

Elements of the $8 \times 8$ determinant after the exponential reduction and certain sealing operations have been performed are given in the following charts. Quantities are still dimensional. 


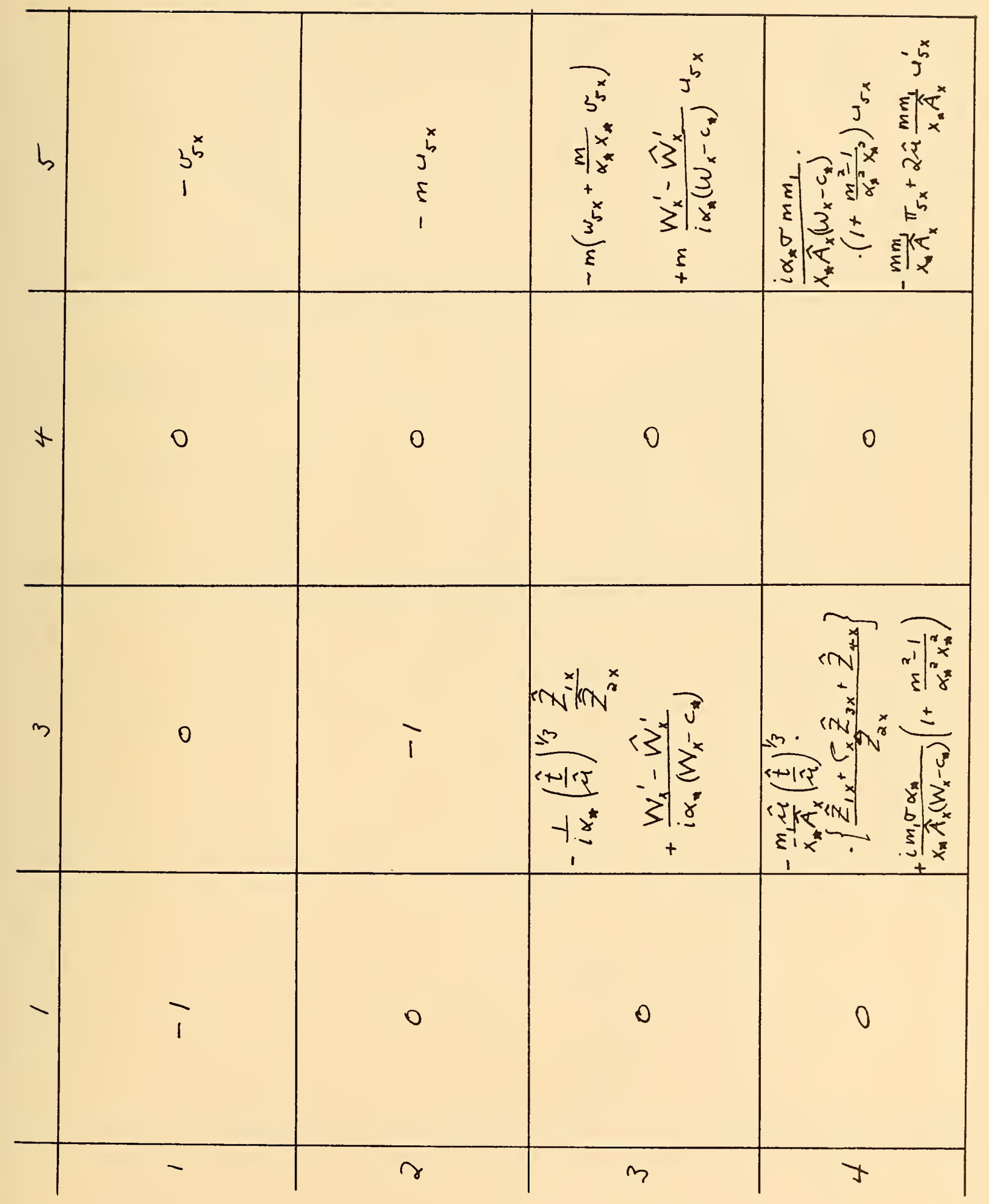




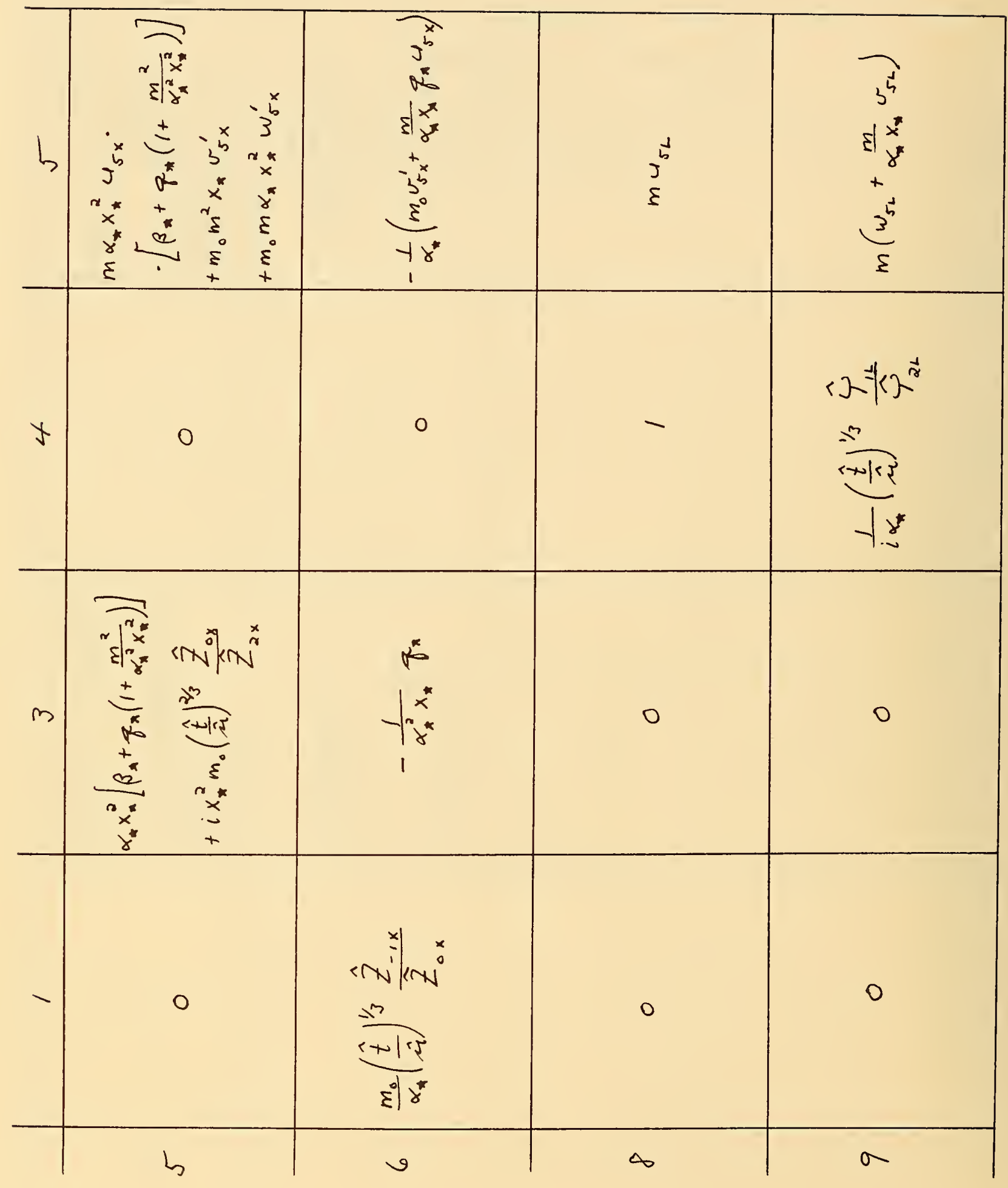




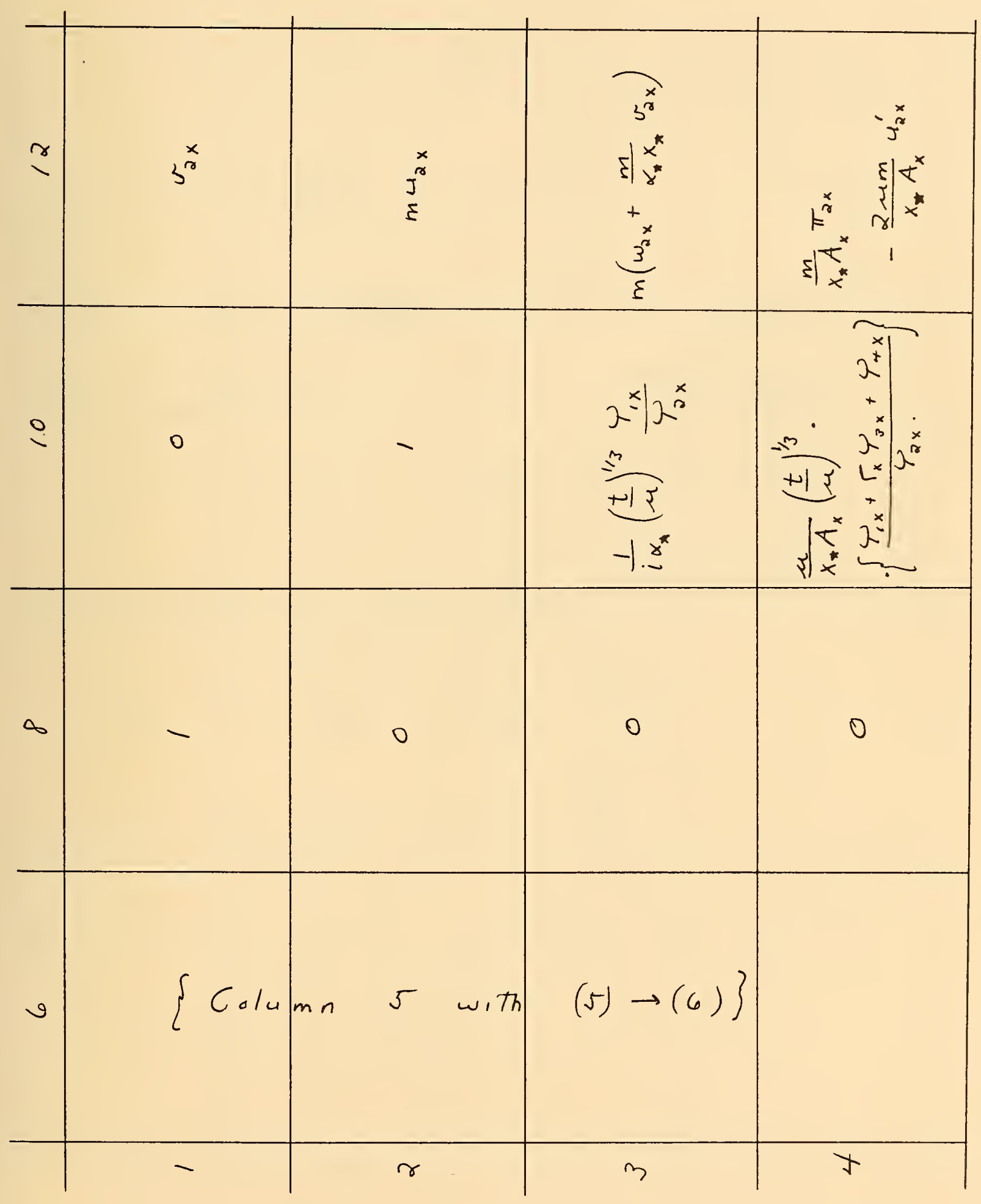




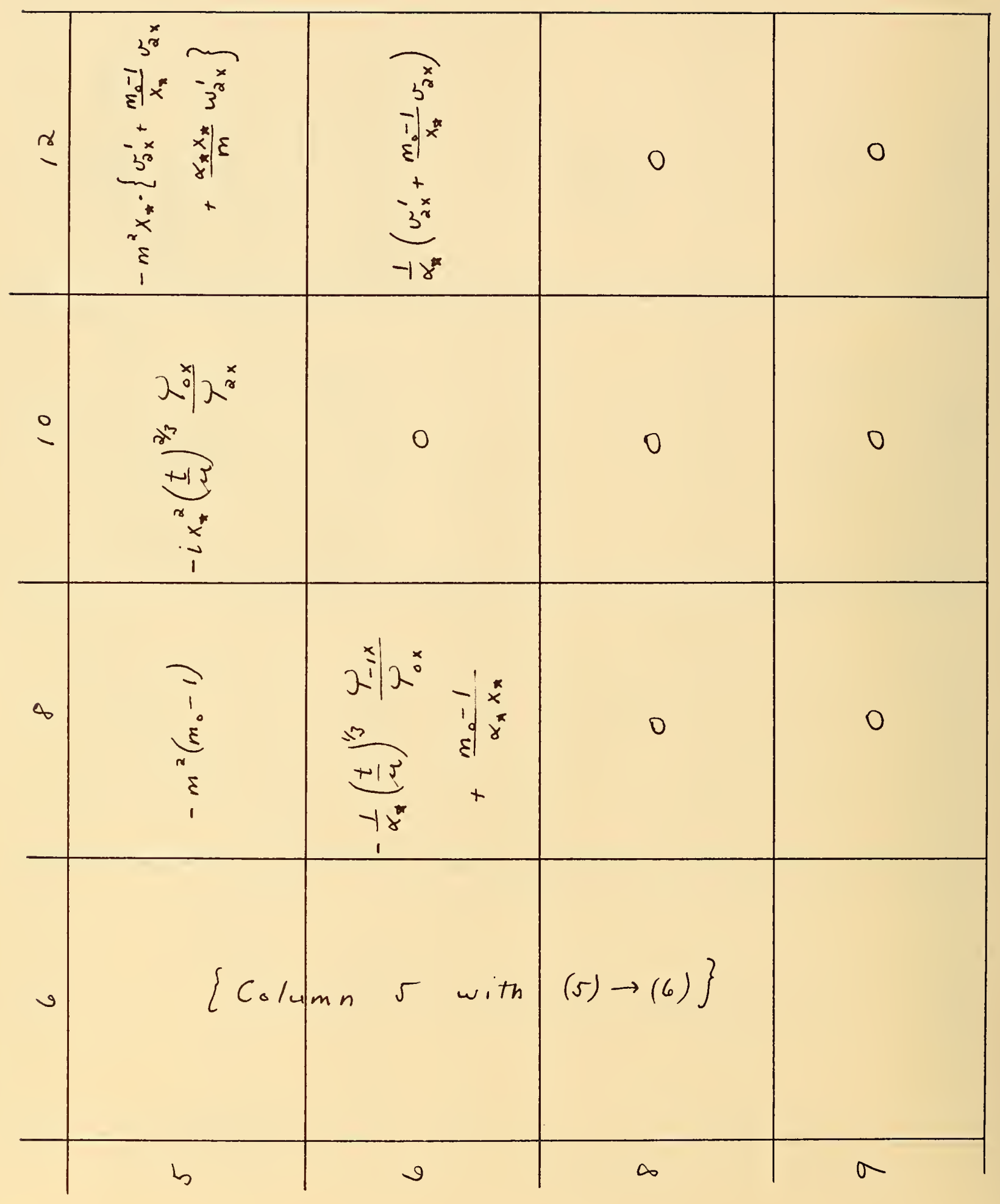




\section{Appendix 3}

Letting $z=z A_{0}^{1 / 2}$, we have the asymptotic expansion:

$$
\left(-\frac{3 \pi}{2} \leqslant \arg z \leqslant \frac{\pi}{2}\right)
$$

$$
\begin{aligned}
& I_{m}(z) \underset{|z|}{\sim} \frac{e^{z}}{(2 \pi z)^{1 / 2}}\left[1+\int_{1 m}\left(\frac{1}{z}\right)\right] \\
&+\frac{e^{-z}}{(2 \pi z)^{1 / 2}} e^{-i\left(m+\frac{1}{2}\right) \pi}\left[1+S_{2 m}\left(\frac{1}{z}\right)\right]
\end{aligned}
$$

where the $S_{j m}$ are asymptotic series in $\frac{1}{z}$ which vanish as $|z| \rightarrow \infty$. Then for some constant $i_{01}$ :

$$
\int_{0}^{z} d s<I_{0}(s)=i_{01}+\frac{z^{1 / 2}}{\sqrt{2} \pi}\left\{\begin{array}{l}
e^{z}\left[1+\int_{10}^{(1)}\left(\frac{1}{z}\right)\right] \\
+i e^{-z}\left[1+\int_{0}^{(1)}\left(\frac{1}{z}\right)\right]
\end{array}\right\}
$$

by integration by parts involving new series $S_{j 0}^{(1)}$.

For $m=0$, we obtain the asymptotic forms

$$
\begin{aligned}
& u \sim B_{1} \frac{\mu^{1 / 4} A_{0}^{-1 / 4}}{\sqrt{2 \pi}}\left(\frac{e^{z}}{r^{1 / 2}}+i \frac{e^{-z}}{r^{1 / 2}}\right) \\
& w \sim B_{3} \frac{\mu^{-1 / 4} A_{0}^{-1 / 4}}{\sqrt{2 \pi}}\left(\frac{e^{z}}{r^{1 / 2}}-i \frac{e^{-z}}{r^{1 / 2}}\right) \\
& u \sim B_{3}\left\{-\frac{i \alpha \mu^{1 / 2} i_{01}}{A_{0} r}-\frac{i \alpha \mu^{1 / 4}}{\sqrt{2 \pi} A_{0}^{3 / 4}}\left(\frac{e^{z}}{r^{1 / 2}}+\frac{i e^{-z}}{r^{1 / 2}}\right)\right\} \\
& \pi \sim B_{5}+L\left(B_{3}\right)
\end{aligned}
$$




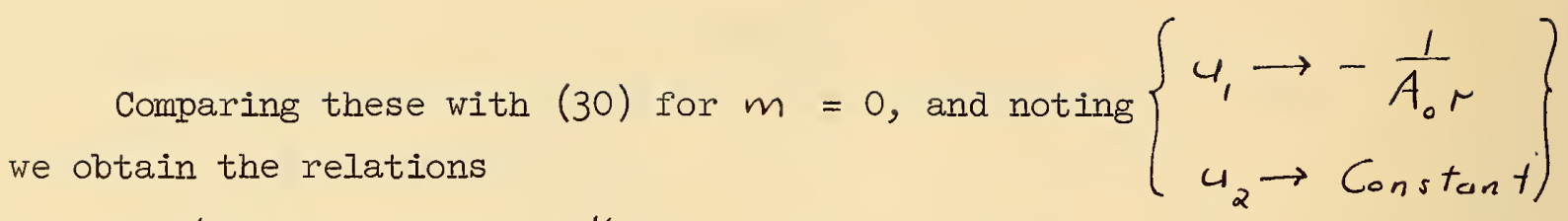

$$
\begin{aligned}
& \frac{P_{0} C_{1}}{A_{0}^{1 / 4}}=B_{1} \frac{\mu^{1 / 4} A_{0}^{-1 / 4}}{\sqrt{2 \pi}}=\frac{C_{2}}{P_{0} A_{0}^{1 / 4}} \\
& \frac{P_{0} C_{3}}{\left(W_{0}-C_{*}\right)^{1 / 2} A_{0}^{1 / 4}}=\frac{B_{3}}{\sqrt{2 \pi} \mu^{1 / 4} A_{0}^{1 / 4}}=-\frac{C_{4}}{P_{0}\left(W_{0}-C_{*}\right)^{1 / 2} A_{0}^{1 / 4}} \\
& -\frac{C_{5}}{A_{0}}=-\frac{i \alpha \mu^{1 / 2} i_{0} B_{3}}{A_{0}} ; \quad C_{6}=B_{5}
\end{aligned}
$$

These provide the three relations (34).

For $m \geq 1$, the matching is a little more difficult. The constants $i_{m, p}$,

$i_{m, p}^{*}, i_{m, p}^{* *}$ are defined by the $|z| \rightarrow \infty$ result: $\left(-\frac{3 \pi}{2} \leqslant \arg z \leqslant \frac{\pi}{2}\right)$

$$
\begin{aligned}
\int_{0}^{z} d \rho e^{p} I_{m}(\rho) \underset{|z|}{\tilde{i_{m p}}} & +\left[\frac{1}{\sqrt{2 \pi}} z^{p-\frac{1}{2}} e^{z}-\frac{1}{\sqrt{2 \pi}} z^{p-\frac{1}{2}} e^{-z} e^{-i \pi\left(m+\frac{1}{2}\right)}\right] \\
+ & {\left[i_{m p}^{*} z^{p-\frac{3}{2}} e^{z}+i_{m p}^{* *} z^{p-\frac{3}{2}} e^{-z}\right] } \\
& +\theta\left(z^{p-\frac{5}{2}} e^{ \pm z}\right)
\end{aligned}
$$

Using this in the relations (32) and their auxiliary relations, we obtain the asymptotic forms

$$
\begin{aligned}
& u \underset{|z|}{\pi}\left[B_{2} z^{m-1}+B_{3} A_{0}^{-1 / 2}\left(i_{m, 1+m} z^{-1-m}-i_{m, 1-m} z^{-1+m}\right)\right] \\
& +z^{-3 / 2} e^{z}\left[B_{3} A_{0}^{-1 / 2}\left(i_{m, 1+m}^{*}-i_{m, 1-m}^{*}\right)\right] \\
& +z^{-3 / 2} e^{-z}\left[B_{3} A_{0}^{-1 / 2}\left(i_{m, 1+m}^{*}-i_{m, 1-m}^{* *}\right)\right] \\
& \omega \tilde{r}_{|z|} \mu^{1 / 2} B_{5}\left[\frac{e^{z}}{(2 \pi z)^{1 / 2}}+\frac{e^{-z} e^{-i \pi\left(m+\frac{1}{2}\right)}}{(2 \pi z)^{1 / 2}}\right]+\theta(u, \pi)
\end{aligned}
$$

56 
For the matching, it suffices to consider the above relations with respect to their counterparts in $(30)$. Since the exponential terms in $4\left(B_{2}, B_{3}\right)$ are correct to a factor $\left(1+\theta\left(\frac{1}{z}\right)\right)$ while $u\left(C_{1}, \ldots, C_{4}\right)$ in (30) is correct to a factor $(1+\theta(r))$, the best match possible to the level of approximation taken is in the leading terms only, $u\left(C_{1}, C_{2}\right)$. The $4\left(C_{3}, C_{4}\right)$ terms are due to coupling with other functions in the $u\left(C_{1}, \ldots, C_{4}\right)$ - approximation and should be suppressed for this match. Then in $w$, the $w\left(C_{1}, C_{2}\right)$ portion is induced by $(w, 4)$ coupling which is the part of $w\left(B_{2}, B_{3}, B_{5}\right)$ not explicitly given. The remaining part, $w\left(C_{3}, C_{4}\right)$, is not coupled to $u$ (the $u\left(C_{3}, C_{4}\right)$ having been suppressed) and should be matched with the free part $w\left(B_{5}\right)$. We obtain then

$$
\begin{aligned}
& -\frac{i m \mu^{1 / 2} P_{0}}{A_{0}^{3 / 4}} C_{1}=B_{3} A_{0}^{-5 / 4} \mu^{3 / 4}\left(i_{m, 1+m}^{*}-i_{m, 1-m}^{*}\right) \\
& -\frac{m \mu^{1 / 2}}{P_{0} A_{0}^{3 / 4}} C_{2}=B_{3} A_{0}^{-5 / 4} \mu^{3 / 4}\left(i_{m, 1+m}^{*}-i_{m, 1-m}^{*}\right)
\end{aligned}
$$

and

$$
\begin{aligned}
& \frac{P_{0}}{\left(W_{0}-c_{n}\right)^{1 / 2} A_{0}^{1 / 4}} C_{3}=\frac{1}{\sqrt{2 \pi}} \mu^{3 / 4} B_{5} \\
& \frac{i}{\left(W_{0}-c_{n}\right)^{1 / 2} A_{0}^{1 / 4} P_{0}} C_{4}=\frac{1}{\sqrt{2 \pi}} A_{0}^{-1 / 4} u^{3 / 4} e^{-i \pi\left(m+\frac{1}{2}\right)} B_{5} .
\end{aligned}
$$

For the inviscid parts, $\left[u\left(B_{j}\right), u_{l}\left(C_{f}\right)\right]$ matching gives, since

$$
\begin{aligned}
& \left\{u_{1} \rightarrow \frac{m}{A_{0}} r^{-m-1}, u_{2} \rightarrow-\frac{m}{A_{0}} r^{m-1}\right\}: \\
& \quad \frac{m}{A_{0}} C_{6}=\mu^{\frac{1+m}{2} A_{0}^{-\left(1+\frac{m}{2}\right)} i_{m, 1+m} B_{3}} \\
& -\frac{m}{A_{0}} C_{5}=\mu^{\frac{1-m}{2}} B_{2}-\mu^{\frac{--m}{2} A_{0}^{-\left(1-\frac{m}{2}\right)} i_{m, 1-m} B_{3} .}
\end{aligned}
$$

Eliminating the $B_{j}$ from the above lead to (33), where

$$
\left(\begin{array}{l}
\gamma^{*} \\
\gamma^{*}
\end{array}\right)=\left(\begin{array}{c}
i_{m, 1+m}^{*}-i_{m, 1-m}^{*} \\
i_{m, 1+m}^{*}-i_{m, 1-m}^{* \star}
\end{array}\right) \text {. }
$$


Appendix 4

TIETJEN'S AND RELATED FUNCTIONS.

In the elements of the determinant $A_{i j}$ occur the Tietjen's function and some related functions incorporating the viscous effects in the so-

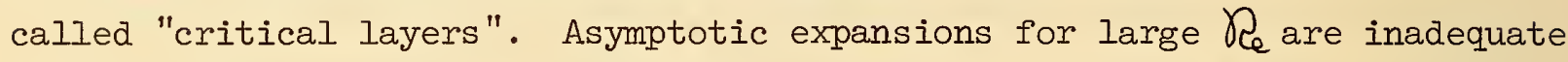
approximations on the neutral stability curves, while the tabulations of some of them given by Holstein [6] do not give adequate accuracy in the crossover range between convergent (small $R$ ) and asymptotic representations. A computing routine was developed to compute these functions taking advantage of a routine written by strand ${ }^{[7]}$ giving Airy's functions $a i(z)$ and $\operatorname{Bi}(z)$ to eight decimal digits.

We introduce the notation:

$$
Q_{p}(z ; B, C) \equiv\left\{\begin{array}{ll}
\frac{z_{p-1}(\rho)}{z_{p}(r)} & (C=1) \\
\varphi_{p-1}(\rho) & (C=-1)
\end{array}\right\}
$$

where

$$
\Gamma=z e^{i \pi\left(1-\frac{B}{2}\right)}, \quad z \geqslant 0 .
$$

Then

$$
\arg \sigma=\left\{\begin{array}{cc}
\frac{\pi}{2} & (B=1) \\
\frac{3 \pi}{2} & (B=-1)
\end{array}\right\} \text {. }
$$

Putting

$$
\begin{array}{ll}
\arg \hat{r}_{x}=\pi\left(1-\frac{B_{1}}{2}\right), & z_{1} \equiv\left|\Gamma_{x}\right|, \\
\operatorname{ang} \hat{r}_{x}=\pi\left(1-\frac{\beta_{2}}{2}\right), & z_{2} \equiv\left|\hat{\Gamma}_{x}\right|, \\
\operatorname{ang} \hat{r}_{L}=\pi\left(1-\frac{B_{3}}{2}\right), & z_{3}=\left|\hat{r}_{L}\right|,
\end{array}
$$

58 
we have:

\begin{tabular}{l|c|c|c} 
& $B_{1}$ & $B_{2}$ & $B_{3}$ \\
\hline$c<0$ & -1 & -1 & -1 \\
$0<c<1$ & -1 & -1 & 1 \\
$c>1$ & 1 & 1 & 1
\end{tabular}

and

$$
\begin{aligned}
& \rho_{x}=i B_{1} z_{1} \\
& \hat{r}_{x}=i B_{2} z_{2} \\
& \hat{r}_{L}=i B_{3} z_{3} .
\end{aligned}
$$

We also have the identity:

$$
\left(\begin{array}{l}
z_{1} \\
T_{1}
\end{array}\right)+r\left(\begin{array}{l}
z_{3} \\
T_{3}
\end{array}\right)+\left(\begin{array}{l}
z_{+} \\
T_{4}
\end{array}\right)=\frac{4}{3}\left(\begin{array}{l}
z_{1} \\
Y_{1}
\end{array}\right)+\frac{2}{3} 5\left(\begin{array}{l}
z_{3} \\
y_{3}
\end{array}\right)
$$

eliminating the need for determining $Z_{+}, Y_{+}$.

Let also

$$
2_{j}(z ; B, C) \equiv i B z Q_{j}(z ; B, C) \text {. }
$$

The elements $A_{i j}$ containing $\left(Z_{j}, Y_{j}\right)$ functions take the following forms:

$$
\begin{aligned}
A_{61}= & \frac{i m_{0}}{\alpha} \frac{\hat{W}_{x}^{\prime}}{1-c} B_{2} z_{2} \mathscr{Z}_{0}\left(z_{2} ; B_{2}, 1\right) \\
A_{33}= & -\frac{\hat{W}_{x}^{\prime}}{(1-c) \alpha} B_{2} z_{2} \mathscr{Z}_{2}\left(z_{2} ; B_{2}, 1\right)+\frac{W_{x}^{\prime}-\hat{W}_{x}^{\prime}}{i \alpha(1-c)} \\
A_{43}=-\frac{2 m_{1}}{x} & \frac{\hat{W}_{x}^{\prime}}{(1-c)^{2} \alpha d} B_{2} z_{2}\left[\frac{2}{3} 2_{2}\left(z_{2} ; B_{2,1}\right)+\frac{i}{3} \frac{B_{2} z_{2}}{2_{3}\left(z_{2} ; B_{2}, 1\right)}\right] \\
& \quad+\frac{m_{1} \sum}{x(1-c)^{2}}\left(1+\frac{m^{2}-1}{\alpha^{2} x^{2}}\right)
\end{aligned}
$$

59 


$$
\begin{aligned}
& A_{53}=\alpha x\left(b+q\left(1+\frac{m^{2}}{\alpha^{2} x^{2}}\right)\right)-i m_{0} x^{2}\left(\frac{\hat{W}_{x}^{\prime}}{1-c}\right)^{2} z_{2}^{2} \mathscr{Z}_{1}\left(z_{2} ; B_{2}, 1\right) \mathscr{Z}_{2}\left(z_{2} ; B_{2}, 1\right) \\
& A_{9+}=-\frac{\hat{W}_{1}^{\prime}}{\alpha c} \cdot B_{3} z_{3} 2_{2}\left(z_{3} ; B_{3},-1\right) \\
& A_{68}=\frac{m_{0}-1}{\alpha x}-\frac{W_{x}^{\prime}}{\alpha(1-c)} i B_{1} z_{1} 2_{0}\left(z_{1} ; B_{1},-1\right) \\
& A_{310}=\frac{W_{x}^{\prime}}{\alpha(1-c)} B_{1} z_{1} 2_{2}\left(z ; B_{1},-1\right) \\
& A_{+10}=\frac{2 m_{1}}{m_{0}} \frac{W_{x}^{\prime}}{\alpha x(1-c)^{2} \gamma d} B_{1} z_{1}\left[\frac{2}{3} Q_{2}\left(z_{1} ; B_{1},-1\right)+\frac{i}{3} \frac{B_{1} z_{1}}{2_{3}\left(z_{1} ; B_{1},-1\right)}\right] \\
& A_{510}=i x^{2}\left(\frac{W_{x}^{1}}{1-c}\right)^{2} z_{1}^{2} 2_{2}\left(z_{1} ; B_{1},-1\right) 2_{1}\left(z_{1} ; B_{1},-1\right) \text {. }
\end{aligned}
$$

The following formulae are readily verified:

$$
\begin{aligned}
Q_{0}(z ; B, C) & \equiv R(z ; B C)+i C I(z ; B C) \\
Q_{0}(z ; 1,1)=\tilde{Q}_{0}(z ; 1,-1) & -e^{i \frac{4 \pi}{3}} \frac{a_{i}{ }^{\prime}\left(z e^{-i \frac{\pi}{6}}\right)}{a_{i}\left(z e^{-i \frac{\pi}{6}}\right)} \\
Q_{0}(z ;-1,1) & =\tilde{Q}_{0}(z ;-1,-1) \\
= & -\frac{a_{i}{ }^{\prime}\left(z e^{i \frac{3 \pi}{2}}\right)+i B_{i}^{\prime}\left(z e^{i \frac{3 \pi}{2}}\right)}{a_{i}\left(z e^{i \frac{3 \pi}{2}}\right)+i B_{l}\left(z e^{i \frac{3 \pi}{2}}\right)}
\end{aligned}
$$

60 
when $R$ and I are real. In addition, we compute the function

$$
X_{3}(z ; B, C)=S(z ; B C)+i C T(z ; B C)
$$

where $\mathrm{S}, \mathrm{T}$ are real. Then with

$$
\begin{aligned}
& X_{2} \equiv\left(1-X_{3}\right) / i B z \\
& X_{1} \equiv\left(Q_{0}-X_{2}\right) / i B z
\end{aligned}
$$

we have may derive:

$$
\begin{aligned}
& Q_{1}=1 / x_{1} \\
& Q_{2}=x_{1} / x_{2} \\
& Q_{3}=2 x_{2} / x_{3} .
\end{aligned}
$$

These are found from the Airy function properties:

$$
\begin{aligned}
& \left(\begin{array}{l}
Z_{p+1} \\
Y_{p+1}
\end{array}\right)=-\left(\begin{array}{l}
Z_{p}^{\prime} \\
Y_{p}^{\prime}
\end{array}\right) \\
& e\left(\begin{array}{l}
Z_{0} \\
Y_{0}
\end{array}\right)=\left(\begin{array}{l}
Z_{-2} \\
Y_{-2}
\end{array}\right)
\end{aligned}
$$

(Recursion formula)

giving

$$
Q_{p}=\frac{p-1}{Q_{p-1} Q_{p-2}-r} \quad(p \geq 2) .
$$

61 
$\chi_{3}(z ; B, C)$ itself is found from the formula:

$$
X_{3}(z ; B, C)=\left(\begin{array}{cc}
\frac{2 Z_{3}}{z_{0}} & (C=1) \\
\frac{2 Y_{3}}{Y_{0}} & (C=-1)
\end{array}\right)
$$

calculating $Z_{3}$ and $Y_{3}$ as follows:

$$
\begin{array}{lll}
\mathrm{BC}=1 & \text { Convergent series: } & 0 \leq z \leq 7.5 \\
& \text { Integral evaluation: } & 7.5<z \leq 13.0 \\
& \text { Asymptotic series: } & z>13.0 \\
\mathrm{BC}=-1 & \text { Convergent series: } & 0 \leq z \leq 10.6 \\
& \text { Asymptotic series: } & z>10.6
\end{array}
$$

Tests indicate a minimum of four significant figures near the joint for $B C=-1$, five significant figures minimum elsewhere. The integrals used, and the basis for the asymptotic expansions, arise from the saddle point method applied to the defining integrals for $\left(Z_{P}, Y_{P}\right)$. They are:

$$
\begin{array}{r}
Z_{p}(5)=e^{\frac{2}{3} \rho^{3 / 2}} r^{-\frac{1+2 p}{4}}(-)^{p} \int_{-\infty}^{\infty} d \sigma e^{-\sigma^{2}} \frac{e^{\sigma 3 / 3 \rho^{3 / 4}}}{\left(1-\frac{\sigma^{3 / 4}}{r^{p}}\right.} \\
Y_{p}(e)=i e^{-\frac{2}{3} e^{3 / 2}} r^{-\frac{1+2 p}{4}} \int_{-\infty}^{\infty} d \sigma e^{-\sigma^{2}} \frac{e^{-i \sigma^{3} / 3 \rho^{3 / 4}}}{\left(1+\frac{i \sigma}{\Gamma^{3 / 4}}\right)^{p}} \\
\left(\frac{\pi}{2} \leqslant \arg r \leqslant \frac{3 \pi}{2}\right)
\end{array}
$$

62 
Appendix 5

THIN FILM APPROXIMATIONS FOR $\left(H, \pi_{5 L}, Q_{5 L}, \pi_{6 L}, Q_{6 L}\right)$.

For the determination of $H=x \frac{Q_{2}(x)}{\pi_{2}(x)}$ from the inviscid pressure equation

$$
\pi^{\prime \prime}+\left(\frac{1}{\rho}-\frac{2 w^{\prime}}{w-c}\right) \pi^{\prime}-\left(\frac{m^{2}}{\rho^{2}}+\alpha^{2}\right) \pi=0
$$

in the core, satisfying $\pi(s) \rightarrow s^{m}$ as $s \rightarrow 0$, we shall break the core into an inner core with $w=w_{0}$ constant for $0 \leq \xi \leq f$ and $m=0$ assumed, and an outer core with linear velocity profile

$w=1+w_{x}^{\prime}(\xi-x)$ for $\xi_{I}<\xi \leq x$ and in which we drop the cylindrical terms $\left(\frac{1}{\rho},{\frac{m}{\xi^{2}}}^{2}\right)$ from the equation. The constants $\xi_{I}$ and $W_{0}$ are determined to give the same gas flow rate as the true profile:

$$
w=1+f\left(x^{2}-\rho^{2}\right)
$$

$$
(0 \leq \rho \leq x)
$$

and $W_{0}=1+W_{x}^{\prime}\left(\rho_{I}-x\right)$

gives continuity in $W$ at $\rho=\rho_{I}$.

$$
\begin{aligned}
& \text { In the inner core, } \\
& \qquad \begin{aligned}
\pi(\xi) & =a I_{0}(\alpha \beta) \\
Q(\xi) & =\pi{ }^{\prime}(\xi)=\alpha a I_{-1}(\alpha \xi) .
\end{aligned}
\end{aligned}
$$

Assuming $\propto \xi_{I}>>I$ as occurs, we shall use leading asymptotic terms at $\xi=\xi_{I}$ :

$$
\begin{aligned}
& \pi\left(\xi_{I}^{-}\right) \doteq \frac{a e^{\alpha \xi_{I}}}{\sqrt{2 \pi \alpha \xi_{I}}} \\
& Q\left(\xi_{I}^{-}\right) \doteq \frac{a \alpha e^{\alpha \xi_{I}}}{\sqrt{2 \pi \alpha \xi_{I}}} .
\end{aligned}
$$

In the outer core, we have:

$$
\Pi^{\prime \prime}-\frac{2}{\rho-\bar{\xi}_{c}} \pi \pi^{\prime}-\alpha^{2} \Pi=0
$$

where $\bar{\xi}_{c}$ is such that

$$
1-c=-w_{x}^{\prime}\left(\bar{\xi}_{c}-x\right) \text {. }
$$

63 
Thus $\bar{\xi}_{c}$ is the critical layer location assuming the same linear profile is valid to $\mathscr{W}=c$ i.e., if $1<c<W_{0}$. If $W(\xi)=c$ in the film, $\bar{\xi}_{c}$ is not the true critical point $\xi_{c}$. Note $\mathscr{W}_{x}^{\prime}=-2 f x$. It is assumed that $\bar{\xi}_{c}>\xi_{I}$. Then

$$
\begin{aligned}
T(p)=A\left(1-\alpha\left(\xi-\bar{\xi}_{c}\right)\right) e^{\alpha\left(\rho-\bar{\rho}_{c}\right)} \\
+B\left(1+\alpha\left(\xi-\bar{\xi}_{c}\right)\right) e^{-\alpha\left(p-\bar{\xi}_{c}\right)} .
\end{aligned}
$$

Joining $\left(\pi, T^{\prime}\right)$ continuously at $\xi^{\prime}=\xi_{I}$, determines the ratio $B / A$ :

$$
\frac{B}{A}=\frac{-e^{2 \alpha\left(\xi_{I}-\bar{\xi}_{c}\right)}}{1+2 \alpha\left(\xi_{I}-\bar{\xi}_{c}\right)} \text {. }
$$

Then we obtain the approximation

$$
H_{1}(x ; \alpha) \equiv \frac{\alpha^{2} \times\left(\bar{\xi}_{c}-x\right)\left[1+\frac{B}{A} e^{\left.-2 \alpha\left(x-\overline{\xi_{c}}\right)\right]}\right.}{\left[1+\alpha\left(\bar{\xi}_{c}-x\right)\right]+\frac{B}{A}\left[1+\alpha\left(x-\bar{\xi}_{c}\right)\right] e^{-2 \alpha\left(x-\bar{\xi}_{c}\right)}} .
$$

The mass flow calculation leads to

$$
\xi_{I}=\frac{x}{4^{1 / 3}} \doteq 0.630 x \text {. }
$$

For the thin film limit $h \rightarrow 0$, another approximation may be made with

$$
\begin{aligned}
& m=\alpha \beta \\
& a=\alpha \sqrt{1+\beta^{2}} .
\end{aligned}
$$

We can, to principal order in $h$, obtain:

$$
T T^{\prime \prime}-\frac{2}{\xi-\bar{\xi}_{c}} \Pi^{\prime}-a^{2} \Pi=0 .
$$

64 
The solution bounded as $\left(\xi-\bar{\xi}_{c}\right) a \rightarrow-\infty$ yields the approximation:

$$
H_{2} \doteq \frac{x a^{2}}{a+\frac{1}{\xi_{c}^{5}-x}}
$$

agreeing with the earlier approximation $H$, when $B e^{-2 \alpha\left(x-\bar{s}_{c}\right)}=0$. Since the terms with $\frac{B}{A}$ are at best approximate the form we have used is the first with $\alpha$ set to $a$,

$$
H=H,(x, a) \text {. }
$$

The approximation is weakest for non-thin films with $\beta>0$. This might be remedied by retaining $m \neq 0$ in the inner core solution, and using the asymptotic expansion associated with $\alpha \rightarrow \infty$ in the solution:

$$
\pi\left(\xi_{I}\right)=a \cdot I_{\alpha \beta}\left(\alpha \xi_{I}\right) \text {. }
$$

These expansions are very complicated and the result would still be approximate in the outer core even if the broken velocity-profile were exact, so this approximation was rejected.

For the liquid film, the profile is again linear, and the thin film case again leads to

$$
\pi^{\prime \prime}-\frac{2}{\xi^{0}-\xi_{c}} \pi^{\prime}-a^{2} \pi=0
$$

as above, where now we have

$$
\xi_{c}=1-c(1-x)
$$

is the critical layer when $0<c<1$.

Setting

$$
\begin{aligned}
& \eta_{x}=x-\xi_{c} \\
& \eta_{1}=1-\xi_{c}
\end{aligned}
$$

and again using

$$
a=\alpha \sqrt{1+\beta^{2}},
$$


the solutions satisfying the requirements:

$$
\begin{aligned}
& \left(\Pi_{5}(x)=1, Q_{5}(x)=0\right) \\
& \left(\Pi_{6}(x)=0, Q_{6}(x)=1\right)
\end{aligned}
$$

give on $\xi=\mathrm{L}$ :

$$
\begin{aligned}
& \left\{\begin{array}{l}
\pi_{\sigma_{L}}=\frac{\eta_{1}}{\eta_{x}}\left[\cosh a\left(\eta_{1}-\eta_{x}\right)-\frac{1}{a \eta_{x}} \sinh a\left(\eta_{1}-\eta_{x}\right)\right] . \\
Q_{5 L}=a \frac{\eta_{1}}{\eta_{x}} \sinh a\left(\eta_{1}-\eta_{x}\right)
\end{array}\right. \\
& \left\{\begin{array}{l}
\pi_{G L}=\frac{a^{2} \eta_{1} \eta_{x}-1}{a^{3} \eta_{x}^{2}} \sinh a\left(\eta_{1}-\eta_{x}\right) \\
+\frac{a\left(\eta_{1}-\eta_{x}\right)}{a^{3} \eta_{x}^{2}} \cos a\left(\eta_{1}-\eta_{x}\right) \\
Q_{G L}=\frac{\eta_{1} \cosh a\left(\eta_{1}-\eta_{x}\right)+\frac{\eta_{1}}{a} \sinh a\left(\eta_{x} \eta_{x}-\eta_{x}\right) .}{} .
\end{array}\right.
\end{aligned}
$$

Special care must be taken in computing $\prod_{6 L}$ when $a\left(\eta_{1}-\eta_{x}\right)$ is small since much cancellation occurs.

These thin film approximations cannot be expected to give good results if gravity causes significant film profile curvature.

66 
Appendix 6

INVISCID LIMIT

As mentioned earlier, the inviscid stability limit may be found by a completely inviscid analysis, or by an order analysis as $\mu \rightarrow 0$, giving the result:

$$
\left|\begin{array}{ccc}
0 & A_{26} & A_{212} \\
A_{45} & A_{+6} & A_{4.2} \\
A_{85} & A_{86} & 0
\end{array}\right|=0
$$

The values of these elements for $\mu=0$ are as follows:

$$
\begin{aligned}
& A_{26}=i x \\
& A_{212}=-i H \\
& A_{45}=-i m_{1} \\
& A_{46}=-\frac{i m_{1} \sum}{(1-c)^{2}}\left(1+\frac{m^{2}-1}{\alpha^{2} x^{2}}\right) \\
& A_{412}=i \frac{1-c}{c} Q_{5 L} \\
& A_{85}=i \times \frac{1-c}{c} Q_{6 L} . \\
& A_{86}=i \times \frac{1}{585} .
\end{aligned}
$$

The result is written simply as :

$$
x+m_{1} H \frac{Q_{6 L}}{Q_{5 L}}-m_{1} H \frac{1}{(1-c)^{2}} \sum \cdot\left(1+\frac{m^{2}-1}{\alpha^{2} x^{2}}\right)=0 .
$$

We use the thin film approximations derived in Appendix 5,

$$
H=\frac{x a^{2}}{a+\frac{2 f x}{1-c}} \text { since } 1-c=-W_{x}^{\prime}\left(\bar{\xi}_{c}-x\right) \text {. }
$$

Noting also

$$
\begin{aligned}
& \eta_{1}=1-\xi_{c}=c h \\
& \eta_{x}=-h(1-c)
\end{aligned}
$$

67 
we have

$$
\begin{aligned}
& Q_{5 L} \doteq-a \frac{c}{1-c} \sinh a_{F} \\
& Q_{6 L} \doteq-\frac{c}{1-c} \cosh a_{F}+\frac{c}{(1-c)^{2} a_{F}} \sinh a_{F}
\end{aligned}
$$

where $a a_{F}=a h$.

Then

$$
\frac{Q_{L L}}{h Q_{r L}}=\frac{\operatorname{cth} a_{F}}{a_{F}}-\frac{1}{(1-c) a_{F}^{2}}
$$

and putting $x \rightarrow 1$,

$$
h H \doteq \frac{a_{F}^{2}}{a_{F}+\frac{2}{1-c}(h f)}
$$

Introducing

$$
\begin{array}{ll}
h f \equiv \frac{m_{0}}{2}\left[1+\frac{m_{1}-1}{2 m_{1}} \frac{g^{2} h^{5}}{\gamma a_{F}} \operatorname{sqn} g^{*}\right] & \text { for } \operatorname{small} h,
\end{array}
$$

we obtain the inviscid stability curve for thin films:

$$
\begin{aligned}
& a_{F}(1-c)^{2}\left(1+m_{1} \operatorname{cthh} a_{F}\right) \\
&-(1-c)\left[m_{1}-m_{0}\left(1+\frac{m_{1}-m_{0}}{2 m_{1}} \frac{g^{2} h^{r}}{\gamma a_{F}}+q g^{*}\right)\right] \\
&-m_{1} a_{F}^{2} \frac{h \sigma}{\gamma a_{F}}\left(1+\beta^{2}-\frac{1}{\alpha^{2}}\right)=0 .
\end{aligned}
$$

(Note that only the disturbance flow is assumed inviscid. The base flow must be viscous.)

68 
When $\sigma=0$, we have the neutral curve

$$
c=1-\frac{m_{1}-m_{0}\left(1+\frac{m_{1}-1}{2 m_{1}} \frac{q^{2} h^{5}}{r_{F}} \operatorname{sqn} g^{*}\right)}{a_{F}\left(1+m_{1} \operatorname{cthh} a_{F}\right)}
$$

which agrees with Feldman's result when $g=0$. When $\sigma \neq 0$, the equation is quadratic. (Feldman's erroneous formulation of the interface equation again leads to a linear equation.) Since concurrent flow implies for the film

$$
\hat{w}^{\prime}(1)<0, \hat{w}^{\prime}(x)<0
$$

we have the restrictions on $g$ :

$$
|g|<\sqrt{\frac{2 m_{1}}{m_{1}-1} k_{F} h^{5}}
$$

for small $h$. With the restriction to concurrent flow then, we have

$$
m_{1}-2 m_{0}<m_{1}-m_{0}\left(1+\frac{m_{1}-1}{2 m_{1}} \frac{g^{2} h^{5}}{k_{F}} \operatorname{sgn} g^{+}\right)<m_{1} \text {. }
$$

Thus in general, since $m_{1}>2 m_{0}$, the sums of the roots $\left(c_{1}, c_{2}\right)$ satisfies

$$
\left(1-c_{1}\right)+\left(1-c_{2}\right)>0
$$

Then for waves not too long such that

$$
\alpha>\frac{1}{\sqrt{1+\beta^{2}}}
$$

the roots $(1-c)$ are of opposite sign: one travels faster than the interface, one slower. For long waves such that

$$
\alpha<\frac{1}{\sqrt{1+\beta^{2}}}
$$

the roots $(I-c)$ are both positive, each wave travelling more slowly than the interface.

For non-long waves, we have for the second solution for small surface 
tension:

$$
c=1+\frac{m_{1} a_{F}^{2} \frac{h \sigma}{\gamma \varepsilon_{F}}\left(1+\beta^{2}-\alpha^{-2}\right)}{m_{1}-m_{0}\left(1+\frac{m_{1}-1}{2 m_{1}} \frac{g^{2} h^{5}}{\gamma R_{F}} \operatorname{sqn} g^{*}\right)} .
$$

70 


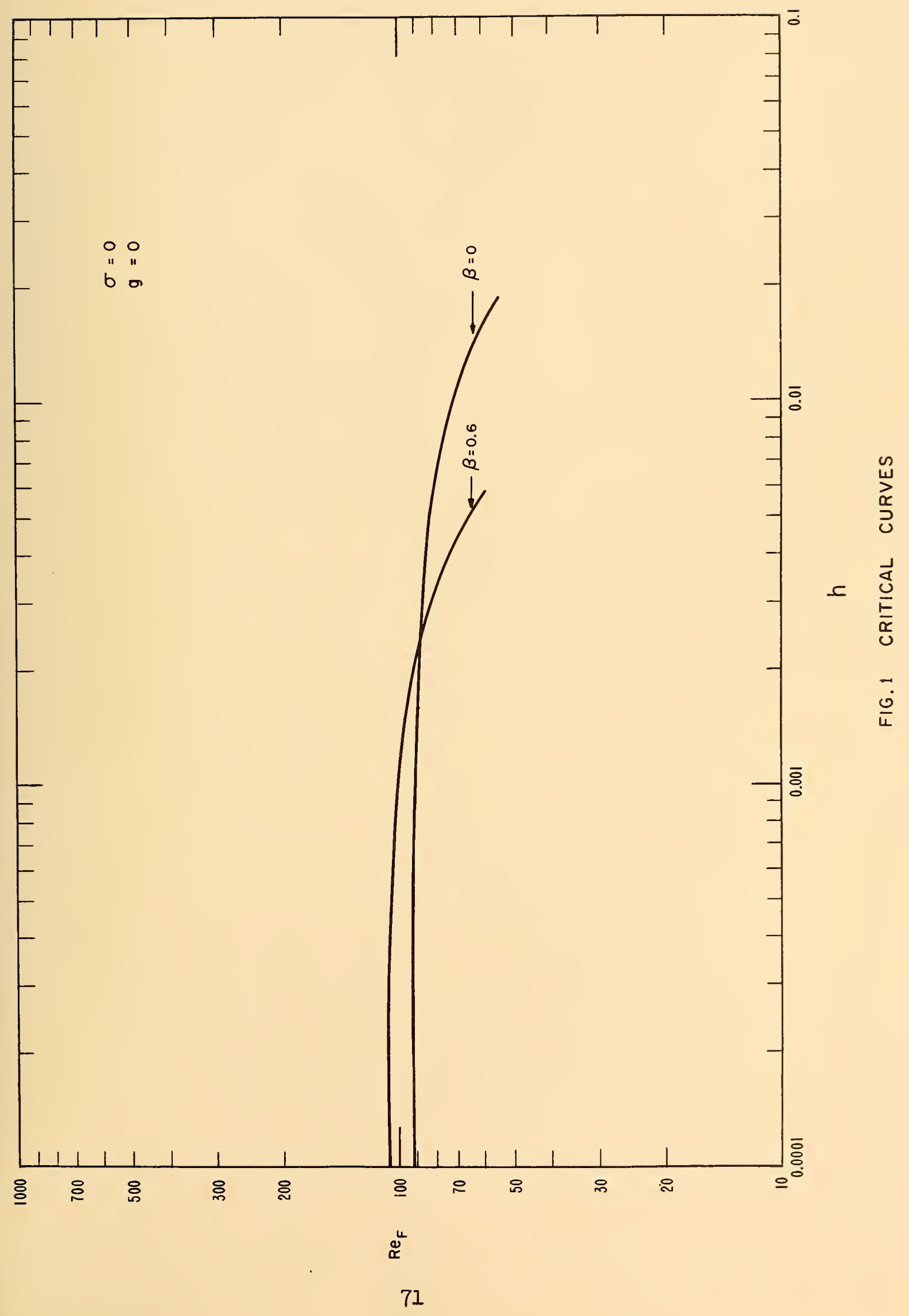





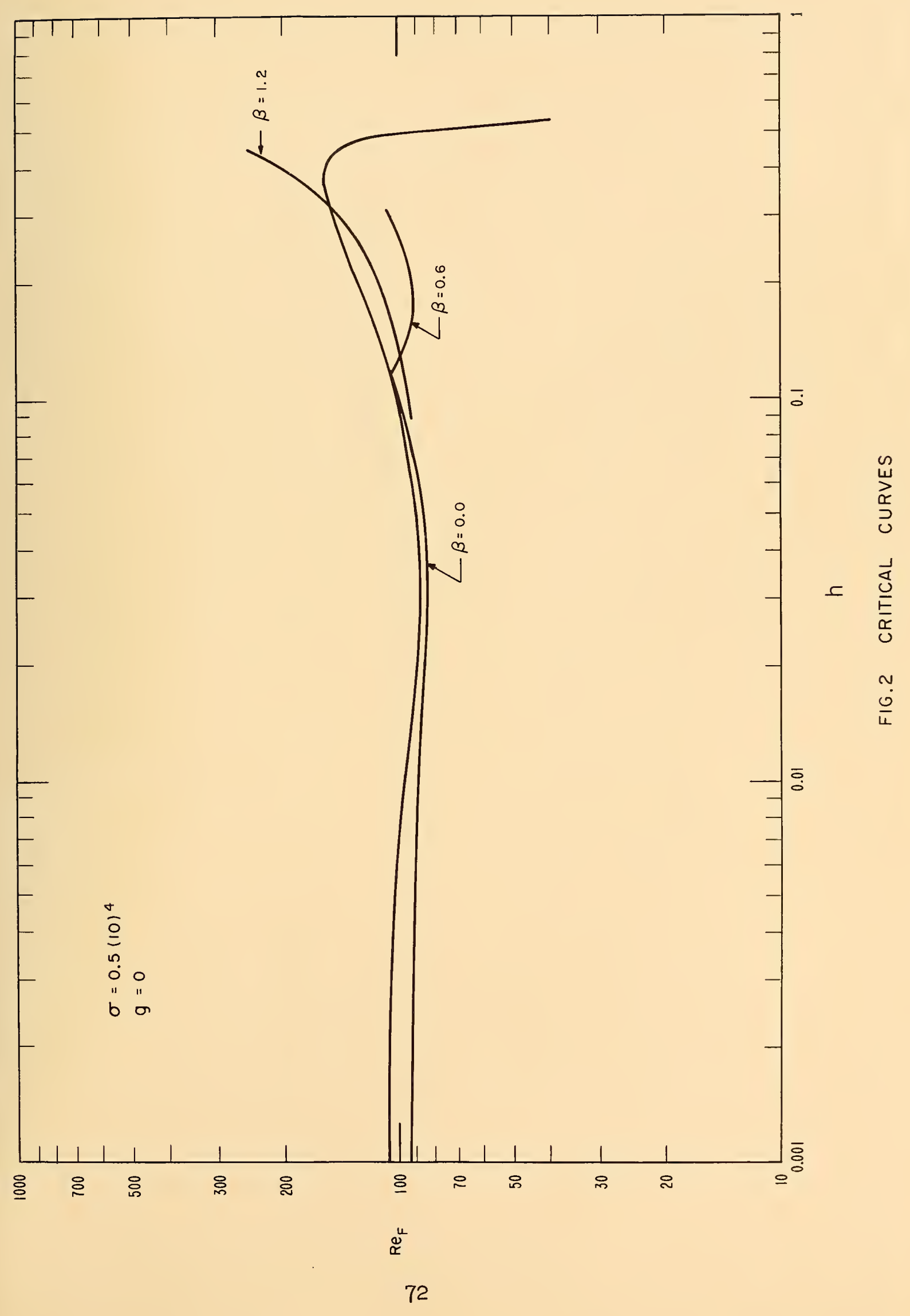





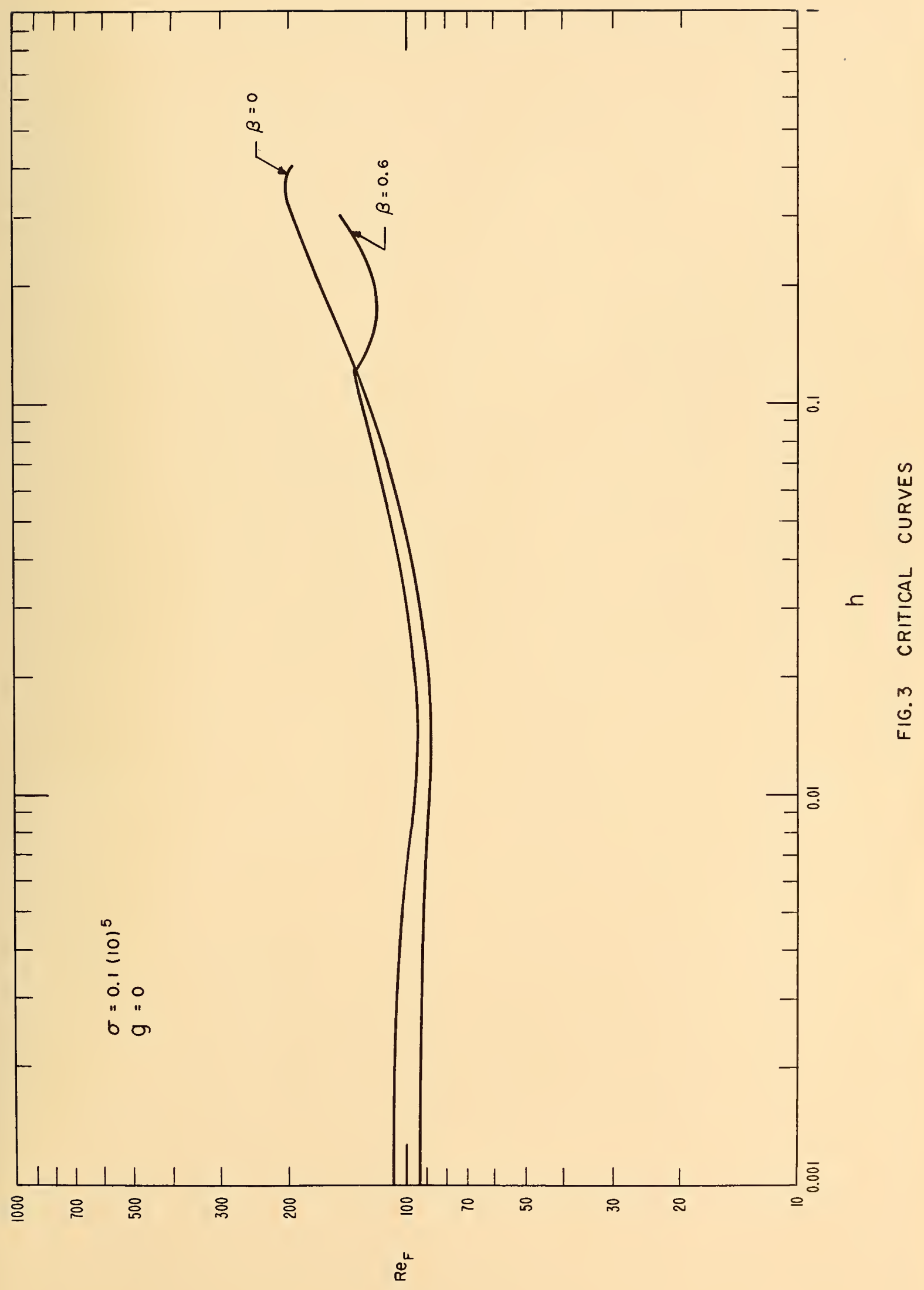





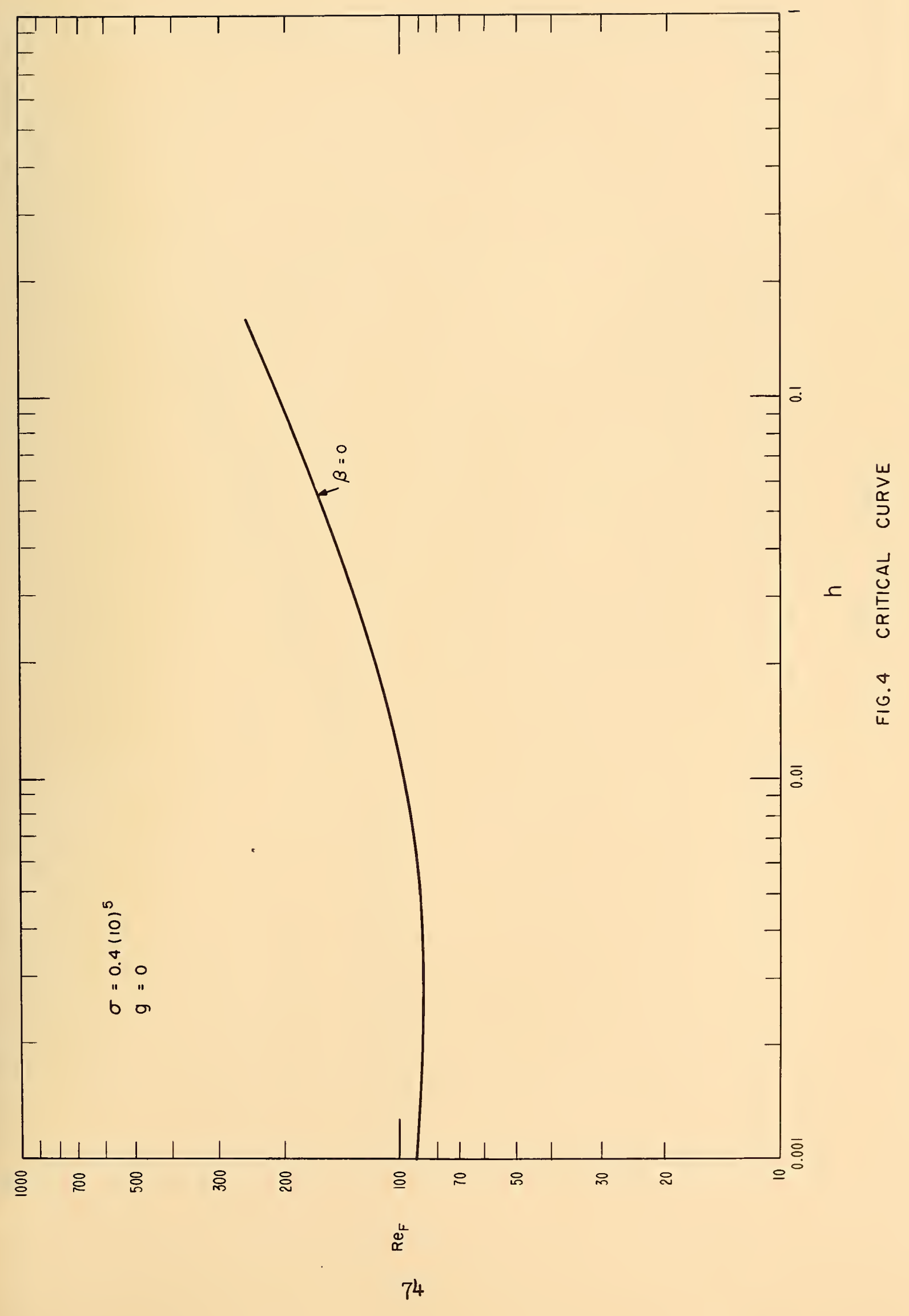




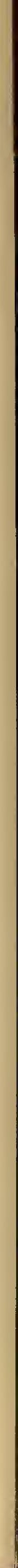




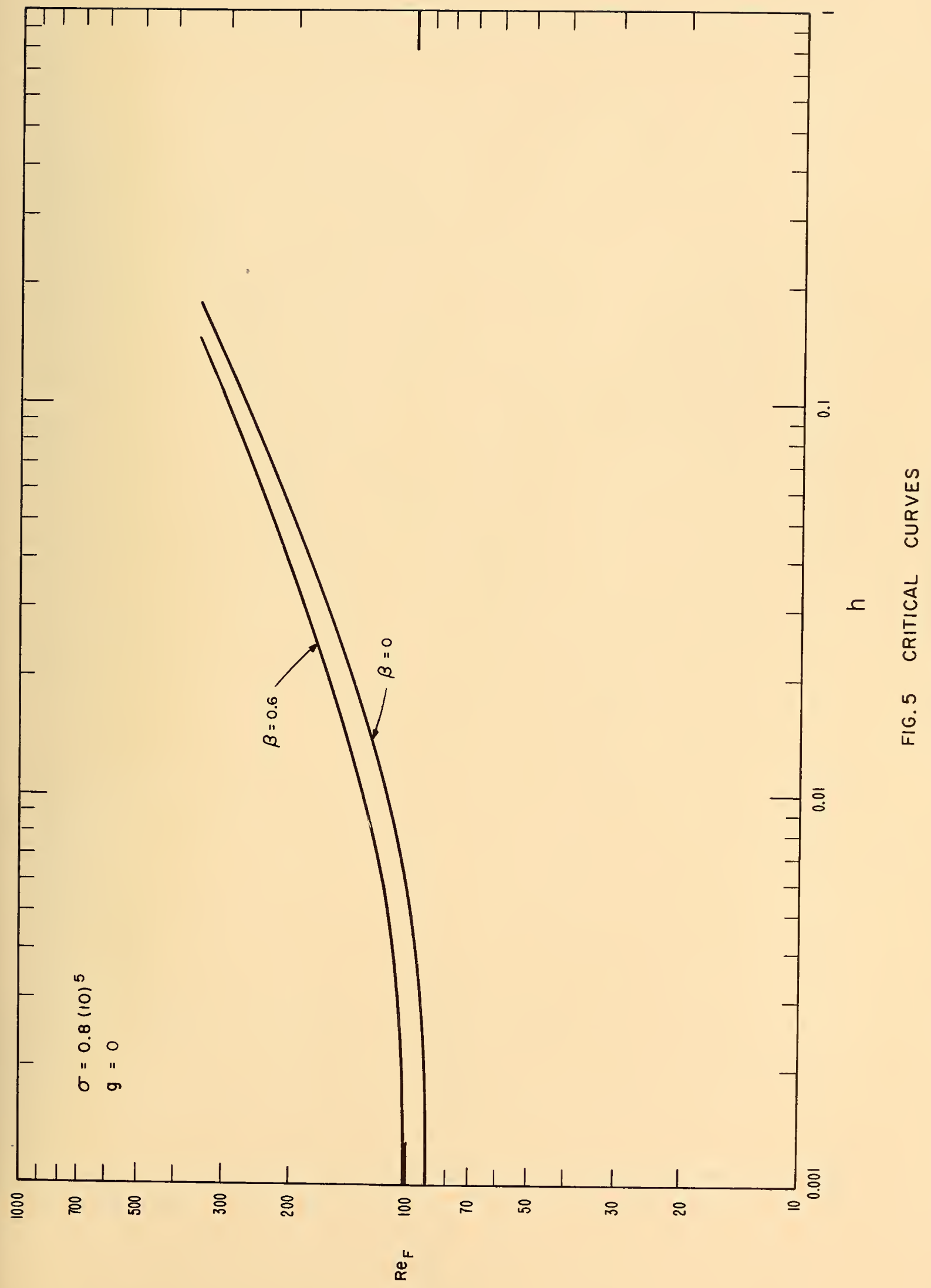




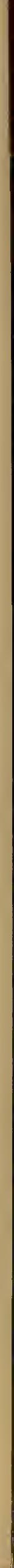




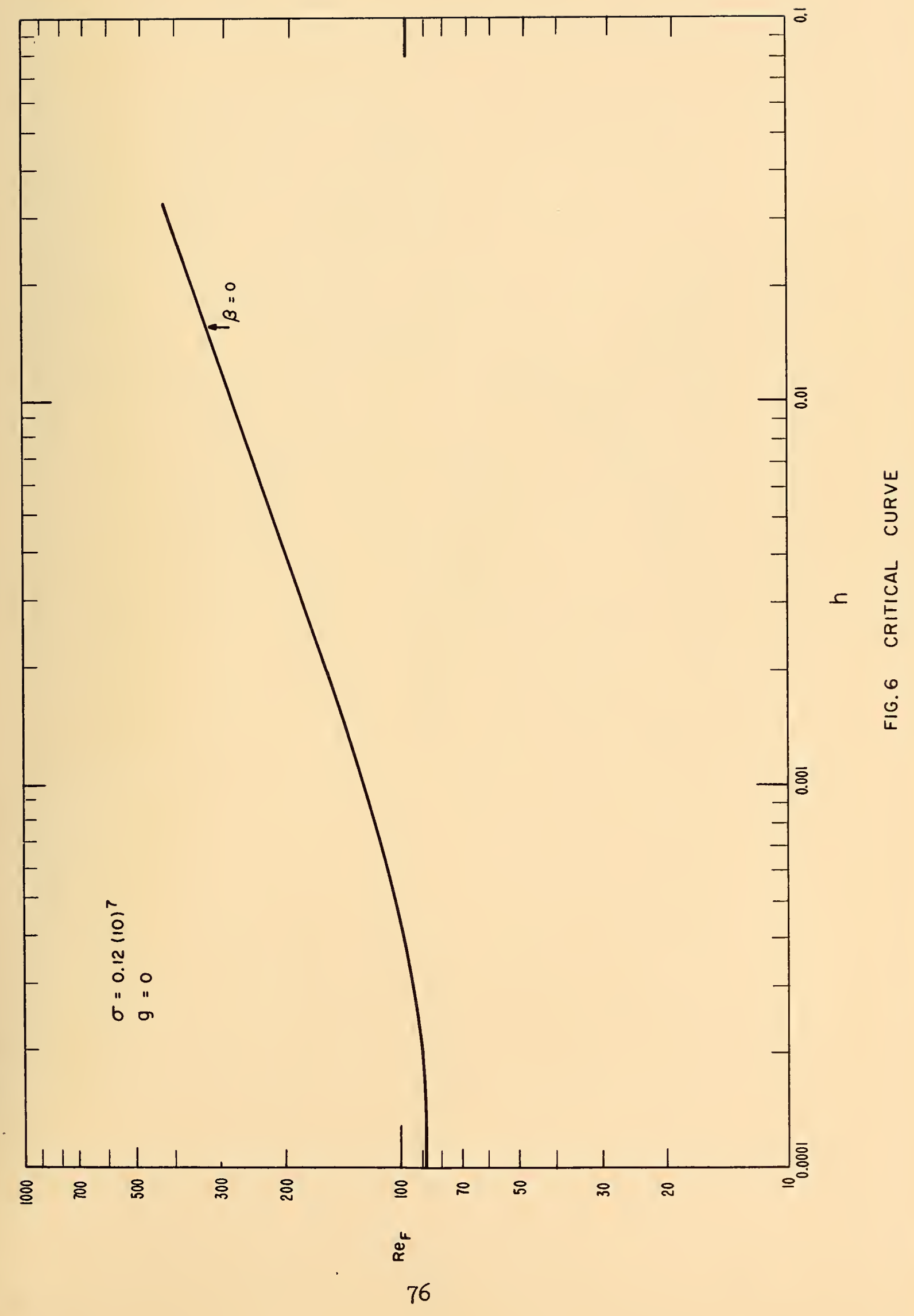





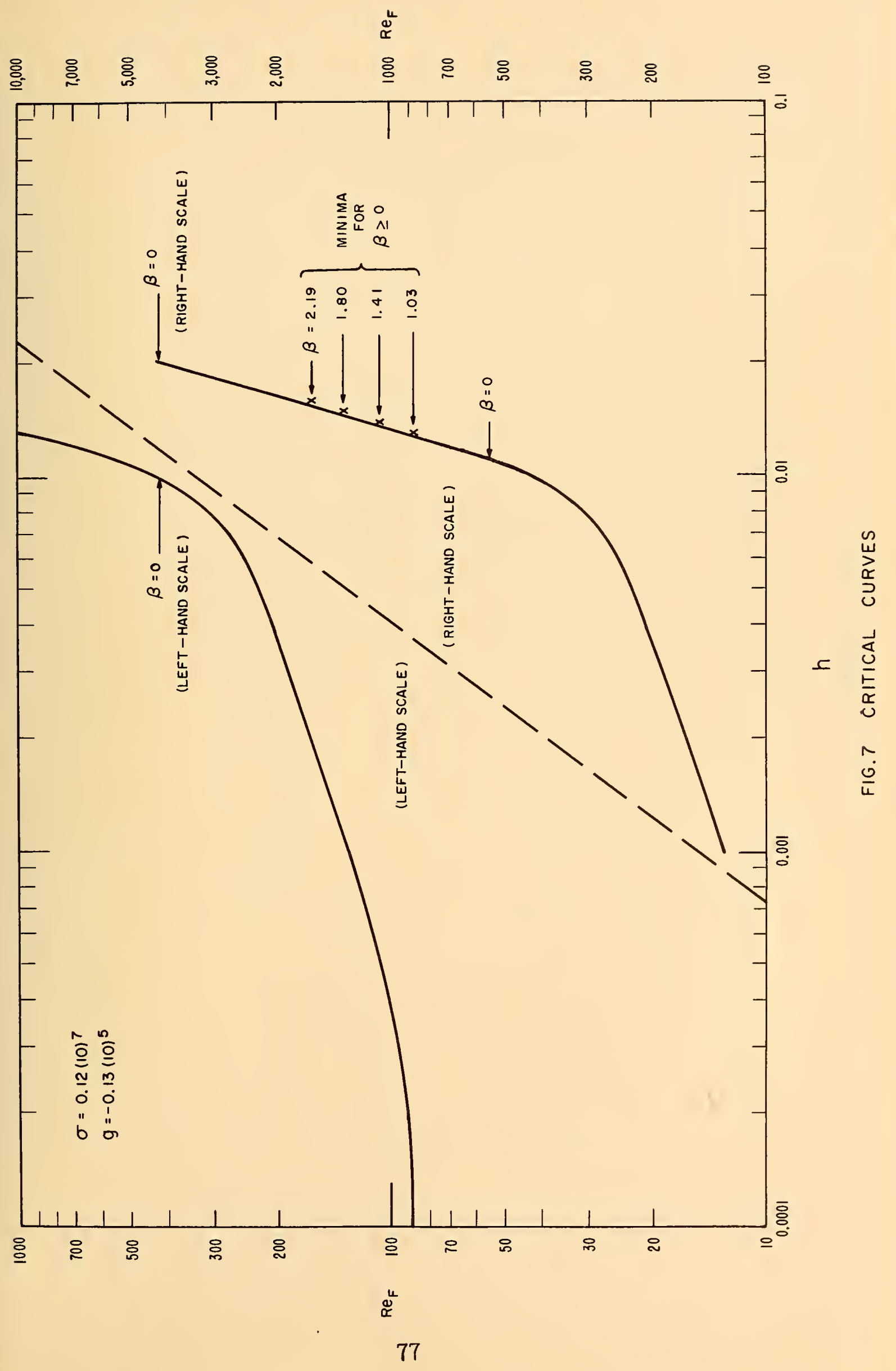





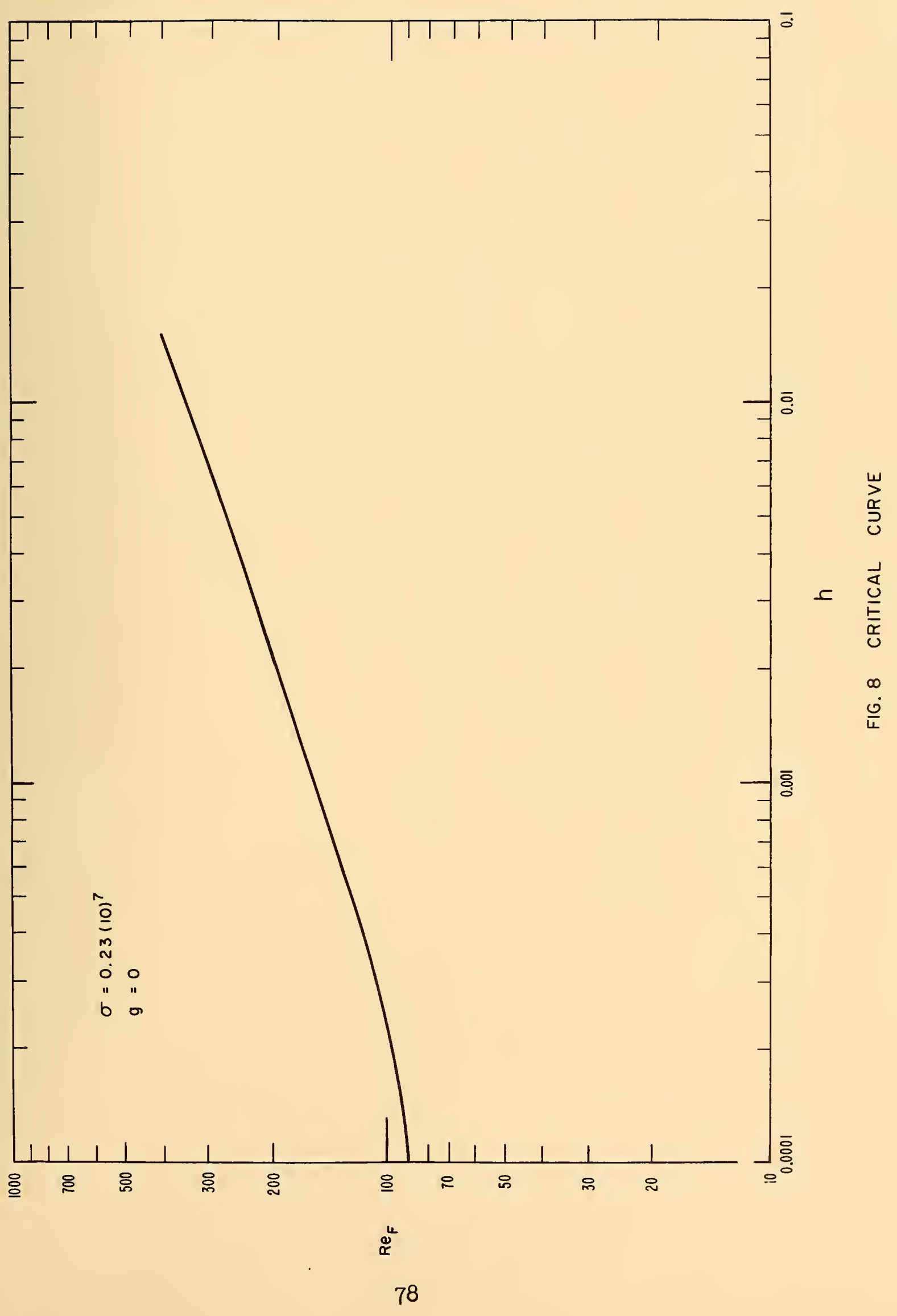



\& \&
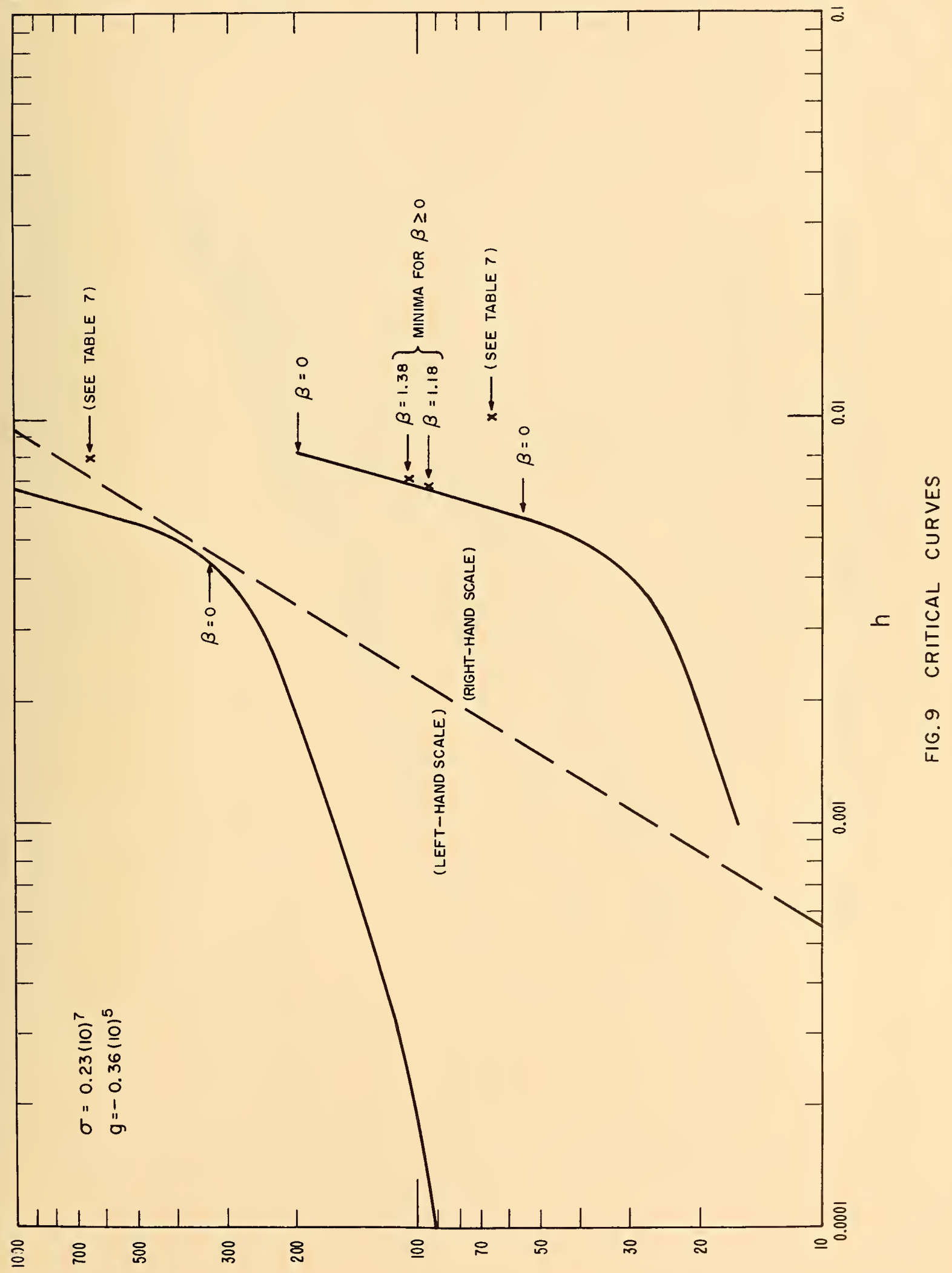

$\dddot{x}^{u}$ 



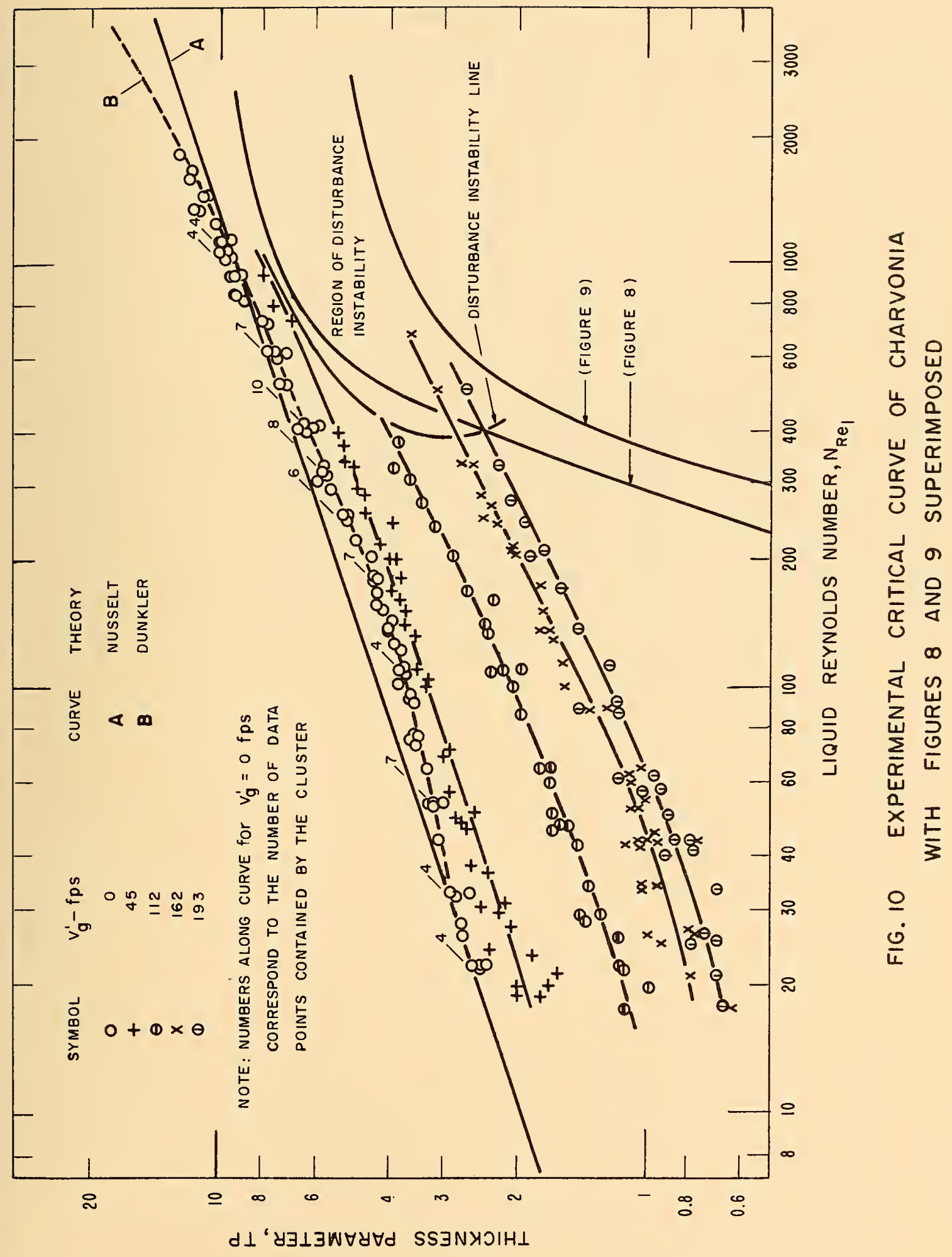




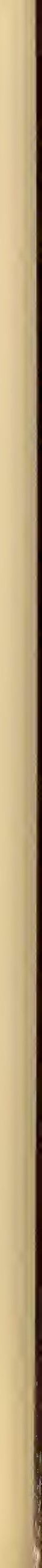


Table 1. $\sigma=0$

\begin{tabular}{|c|c|c|c|c|c|c|c|c|c|c|c|c|}
\hline$g$ & $\beta$ & $h$ & $\left(R_{F}\right)_{C_{0}}$ & $\alpha_{F}$ & $c$ & $\frac{\partial c_{I}}{\partial R_{F}}$ & $\frac{\partial R_{F}}{\partial h}$ & $\frac{\partial R_{E}}{\partial m_{0}}$ & $\begin{array}{l}\partial \mathrm{Bu}_{F_{1}} \\
\partial \mathrm{m}_{1}\end{array}$ & $\begin{array}{l}\partial R_{F} \\
\partial \sigma\end{array}$ & $\frac{\partial R_{F}}{\partial g}$ & $\frac{\partial R_{F}}{\partial \beta}$ \\
\hline \multirow[t]{24}{*}{0} & \multirow[t]{14}{*}{0} & .00100 & 93.5 & 1.91 & .583 & $.42(-3)$ & $-.60(+2)$ & $.22(+1)$ & $-.66(-1)$ & & & \\
\hline & & .00432 & 92.9 & 2.01 & .589 & $.33(-3)$ & $-.22(+3)$ & $.27(+1)$ & $-.80(-1)$ & & & \\
\hline & & .00898 & 91.9 & 2.01 & .589 & $.35(-3)$ & $-.22(+3)$ & $.26(+1)$ & $-.78(-1)$ & & & \\
\hline & & .0154 & 90.5 & 2.01 & .588 & $.37(-3)$ & $-.20(+3)$ & $.24(+1)$ & $-.71(-1)$ & & & \\
\hline & & .0227 & 89.1 & 2.01 & .588 & $.39(-3)$ & $-.19(+3)$ & $.22(+1)$ & $-.607(-1)$ & & & \\
\hline & & .0320 & 87.3 & 2.01 & .587 & $.43(-3)$ & $-.17(+3)$ & $.20(+1)$ & $-.60(-1)$ & & & \\
\hline & & .0428 & 85.2 & 2.12 & .592 & $.37(-3)$ & $-.21(+3)$ & $.22(+1)$ & $-.68(-1)$ & & & \\
\hline & & .0546 & 82.6 & 2.12 & .591 & $.42(-3)$ & $-.11(+3)$ & $.19(+1)$ & $-.58(-1)$ & & & \\
\hline & & .0697 & 79.8 & 2.12 & .590 & $.48(-3)$ & $-.17(+3)$ & $.16(+1)$ & $-.49(-1)$ & & & \\
\hline & & .0848 & 76.5 & 2.23 & .594 & $.44(-3)$ & $-.19(+3)$ & $.17(+1)$ & $-.49(-1)$ & & & \\
\hline & &.$\overline{103}$ & 72.9 & 2.34 & .598 & $.42(-3)$ & $-.21(+3)$ & $.16(+1)$ & $-.49(-1)$ & & & \\
\hline & & .119 & 69.5 & 2.34 & .595 & $.52(-3)$ & $-.21(+3)$ & $.12(+1)$ & $-.39(-1)$ & & & \\
\hline & & .138 & 65.5 & 2.47 & .598 & $.51(-3)$ & $-.19(+3)$ & . $11(+1)$ & $-.34(-1)$ & & & \\
\hline & & .160 & 60.7 & 2.59 & .597 & $.64(-3)$ & $-.12(+3)$ & $.69(0)$ & $-.21(-1)$ & & & , \\
\hline & .2 & .00100 & 95.2 & 1.90 & .585 & $.38(-3)$ & & $.22(+1)$ & $-.69(-1)$ & $-.51(-4)$ & & $.21(+2)$ \\
\hline & .4 & .00100 & 100. & 1.81 & .585 & $.36(-3)$ & & $.24(+1)$ & $-.74(-1)$ & $-.56(-4)$ & & $.40(+2)$ \\
\hline & \multirow[t]{8}{*}{.6} & .00100 & 109. & 1.72 & .589 & $.28(-3)$ & & $.30(+1)$ & $-.94(-1)$ & $-.80(-4)$ & & $.74(+2)$ \\
\hline & & .00432 & 106. & 1.72 & .589 & $.31(-3)$ & $-.74(3)$ & $.27(+1)$ & $-.86(-1)$ & $-.32(-3)$ & $.50(-3)$ & $.59(2)$ \\
\hline & & .00898 & 102. & 1.72 & .588 & $.35(-3)$ & $-.70(3)$ & $.23(1)$ & $-.76(-1)$ & $-.58(-3)$ & $.94(-3)$ & $.41(2)$ \\
\hline & & .0154 & 97.2 & 1.81 & .594 & $.33(-3)$ & $-.81(3)$ & $.24(1)$ & $-.81(-1)$ & $-.13(-2)$ & $.19(-2)$ & $.40(2)$ \\
\hline & & .0227 & 90.9 & 1.81 & .593 & $.42(-3)$ & $-.73(3)$ & $.18(1)$ & $-.66(-1)$ & $-.14(-2)$ & $.24(-2)$ & $.12(2)$ \\
\hline & & .0319 & 83.0 & 1.90 & .596 & $.46(-3)$ & $-.78(3)$ & $.14(1)$ & $-.61(-1)$ & $-.19(-2)$ & $.36(-2)$ & $-.56(1)$ \\
\hline & & .0428 & 73.4 & 1.99 & .598 & $.58(-3)$ & $-.79(3)$ & $.84(0)$ & $-.51(-1)$ & $-.12(-2)$ & $.48(-2)$ & $-.31(2)$ \\
\hline & & .0546 & 63.2 & 2.09 & .597 & $.85(-3)$ & $-.70(3)$ & $.20(0)$ & $-.37(-1)$ & $.24(-2)$ & $.53(-2)$ & $-.50(2)$ \\
\hline
\end{tabular}




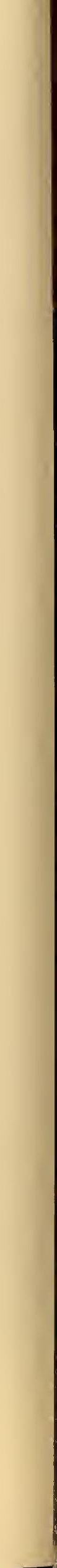




\begin{tabular}{|c|c|c|c|c|c|c|c|c|c|c|c|c|}
\hline 9 & $\beta$ & $h$ & $\left(R_{p}\right)_{m}$ & $\alpha_{F}$ & $c$ & $\begin{array}{l}\text { able } 2 . \\
\frac{\partial c_{I}}{\partial R_{F}}\end{array}$ & $\begin{array}{l}=0.5(1 \\
\frac{\partial R_{F}}{\partial n}\end{array}$ & $\frac{\partial R_{B}}{\partial m_{0}}$ & $\frac{\partial R_{p}}{\partial m_{1}}$ & $\frac{\partial R_{p}}{\partial \sigma}$ & $\frac{\partial R_{B}}{\partial g}$ & $\frac{\partial R_{p}}{\partial \beta}$ \\
\hline \multirow[t]{45}{*}{0} & \multirow[t]{27}{*}{0} & .00432 & 91.6 & 2.00 & .586 & $.35(-3)$ & $-.43(3)$ & $.23(1)$ & $-.72(-1)$ & $-.23(-3)$ & $.49(-3)$ & $.42(1)$ \\
\hline & & .00898 & 89.5 & 2.00 & .582 & $.38(-3)$ & $-.34(3)$ & $.20(1)$ & $-.62(-1)$ & $-.34(-3)$ & $.92(-3)$ & $.24(1)$ \\
\hline & & .0154 & $87 \cdot 3$ & 2.10 & .581 & $.34(-3)$ & $-.31(3)$ & $.21(1)$ & $-.64(-1)$ & $-.44(-3)$ & $.19(-2)$ & $.23(1)$ \\
\hline & & .0227 & 85.4 & 2.10 & .574 & $.40(-3)$ & $-.13(3)$ & $.16(1)$ & $-.47(-1)$ & $+.34(-4)$ & $.21(-2)$ & $.53(0)$ \\
\hline & & .0320 & 84.9 & 2.10 & .567 & $.47(-3)$ & $+.46(2)$ & $.11(1)$ & $-.32(-1)$ & $.10(-2)$ & $.25(-2)$ & $-.19(0)$ \\
\hline & & .0428 & 86.1 & 2.10 & .559 & $.54(-3)$ & $.17(3)$ & $.74(0)$ & $-.21(-1)$ & $.22(-2)$ & $.22(-2)$ & $-.35(0)$ \\
\hline & & .0546 & 88.7 & 2.10 & .553 & $.59(-3)$ & $.25(3)$ & $.50(0)$ & $-.13(-1)$ & $.36(-2)$ & $.25(-2)$ & $+.16(0)$ \\
\hline & & .0697 & 92.6 & 2.00 & .543 & $.70(-3)$ & $.26(3)$ & $.26(0)$ & $-.61(-2)$ & $.42(-2)$ & $.15(-2)$ & $-.54(0)$ \\
\hline & & .0848 & 96.9 & 1.91 & .536 & $.77(-3)$ & $.25(3)$ & $.14(0)$ & $-.20(-2)$ & $.49(-2)$ & $.18(-2)$ & $-.12(1)$ \\
\hline & & .103 & 102. & 1.91 & .531 & $.77(-3)$ & $.26(3)$ & $.68(-3)$ & $+.24(-2)$ & $.59(-2)$ & $.76(-3)$ & $-.37(0)$ \\
\hline & & .119 & 107. & 1.82 & .524 & $.80(-3)$ & $.24(3)$ & $-.70(-1)$ & $.49(-2)$ & $.66(-2)$ & $.19(-2)$ & $-.11(1)$ \\
\hline & & .138 & 112. & 1.82 & .521 & $.78(-3)$ & $.25(3)$ & $-.17(0)$ & $.81(-2)$ & $.73(-2)$ & $-.10(-3)$ & $-.28(0)$ \\
\hline & & .160 & 118. & 1.73 & .514 & $.79(-3)$ & $.23(3)$ & $-.24(0)$ & . $11(-1)$ & $.84(-2)$ & $+.29(-2)$ & $-.95(0)$ \\
\hline & & .185 & 124. & 1.65 & .508 & $.77(-3)$ & $.21(3)$ & $-.32(0)$ & $.13(-1)$ & $.81(-2)$ & $-.32(-3)$ & $-.17(1)$ \\
\hline & & .214 & 131. & 1.65 & .503 & $.73(-3)$ & $.20(3)$ & $-.44(0)$ & $.17(-1)$ & $.98(-2)$ & $+.10(-2)$ & $+.24(0)$ \\
\hline & & .248 & 139. & 1.57 & .496 & $.69(-3)$ & $.18(3)$ & $-.54(0)$ & $.20(-1)$ & $.95(-2)$ & $-.24(-2)$ & $.40(0)$ \\
\hline & & .273 & 144. & 1.57 & .492 & $.65(-3)$ & $.16(3)$ & $-.63(0)$ & $.23(-1)$ & $.11(-1)$ & $.69(-3)$ & $\cdot 31(1)$ \\
\hline & & .301 & 149. & 1.50 & .484 & $.61(-3)$ & $.11(3)$ & $-.70(0)$ & $.26(-1)$ & $.10(-1)$ & $-.37(-2)$ & $.35(1)$ \\
\hline & & .332 & 153. & 1.49 & .477 & $.56(-3)$ & $.69(2)$ & $-.79(0)$ & $.29(-1)$ & $.12(-1)$ & $-.25(-3)$ & $.86(1)$ \\
\hline & & .366 & 156. & 1.37 & .463 & $.54(-3)$ & $.74(0)$ & $-.96(0)$ & $.34(-1)$ & $.13(-1)$ & $+.50(-2)$ & $.40(2)$ \\
\hline & & .404 & 155. & 1.37 & .447 & $.47(-3)$ & $-.14(3)$ & $-.10(1)$ & $.36(-1)$ & $.74(-2)$ & $-.18(-1)$ & $.80(2)$ \\
\hline & & .445 & 143. & 1.30 & .415 & $.39(-3)$ & $-.47(3)$ & $-.10(1)$ & $.29(-1)$ & $-.15(0)$ & $-.60(0)$ & $.13(3)$ \\
\hline & & .491 & 102. & 1.24 & .326 & $.39(-3)$ & $-.11(4)$ & $-.20(0)$ & $.44(-2)$ & $-.51(-1)$ & $-.12(0)$ & $.21(3)$ \\
\hline & & .501 & 78.8 & 1.24 & .264 & $.76(-3)$ & $-.70(3)$ & $.55(0)$ & $.21(-1)$ & $.28(1)$ & $.55(1)$ & $.49(2)$ \\
\hline & & .511 & 63.8 & 1.34 & .197 & $.78(-3)$ & $-.88(3)$ & $-.43(-1)$ & $-.16(-2)$ & $.30(-2)$ & $.16(-1)$ & $.76(2)$ \\
\hline & & $\overline{.521}$ & 52.6 & 1.37 & .123 & $.13(-2)$ & $-.96(3)$ & $-.14(0)$ & $+.33(-4)$ & $-.13(0)$ & $-.19(0)$ & $.13(3)$ \\
\hline & & .532 & 43.2 & 1.40 & .050 & $.20(-2)$ & $=.44(4)$ & $-.12(2)$ & $-.69(0)$ & $-.13(3)$ & $-.16(3)$ & $.80(2)$ \\
\hline & \multirow[t]{18}{*}{.6} & .00432 & 104 & 1.73 & .586 & $.31(-3)$ & $-.10(4)$ & $.26(1)$ & $-.81(-1)$ & $-.27(-3)$ & $.49(-3)$ & $.54(2)$ \\
\hline & & .00898 & 99.6 & 1.73 & .582 & $.38(-3)$ & $-.81(3)$ & $.20(1)$ & $-.65(-1)$ & $-.34(-3)$ & $.87(-3)$ & $.32(2)$ \\
\hline & & .0154 & 93.4 & 1.82 & .579 & $.39(-3)$ & $-.67(3)$ & $.16(1)$ & $-.55(-1)$ & $-.14(-3)$ & $.15(-2)$ & $.27(2)$ \\
\hline & & .0227 & 89.1 & 1.91 & .574 & $.44(-3)$ & $-.34(3)$ & $.10(1)$ & $-.39(-1)$ & $+.10(-2)$ & $.21(-2)$ & $.32(2)$ \\
\hline & & .0320 & 87.4 & 1.82 & .561 & $.70(-3)$ & $-.17(2)$ & $.35(0)$ & $-.18(-1)$ & $.22(-2)$ & $.14(-2)$ & $.16(2)$ \\
\hline & & .0428 & 88.3 & 1.82 & .551 & $.87(-3)$ & $+.18(3)$ & $-.11(-1)$ & $-.72(-2)$ & $.40(-2)$ & $.13(-2)$ & $.26(2)$ \\
\hline & & .0546 & 90.8 & 1.73 & .541 & $.11(-2)$ & $.23(3)$ & $-.24(0)$ & $-.14(-2)$ & $.50(-2)$ & $.72(-3)$ & $.26(2)$ \\
\hline & & .0697 & 94.6 & 1.65 & .531 & $.13(-2)$ & $.25(3)$ & $-.45(0)$ & $+.33(-2)$ & $.62(-2)$ & $.39(-3)$ & $.29(2)$ \\
\hline & & .0848 & 98.1 & 1.50 & .521 & $.14(-2)$ & $.21(3)$ & $-.64(0)$ & $.45(-2)$ & $.67(-2)$ & $-.36(-4)$ & $.21(2)$ \\
\hline & & .103 & 102 . & 1.29 & .509 & $.15(-2)$ & $.13(3)$ & $-.96(0)$ & $.33(-2)$ & $.74(-2)$ & $-.52(-3)$ & $.14(1)$ \\
\hline & & .119 & 103. & 1.12 & .498 & $.15(-2)$ & $.48(2)$ & $-.14(1)$ & $.88(-3)$ & $.82(-2)$ & $-.14(-2)$ & $-.25(2)$ \\
\hline & & .138 & 95.6 & .592 & .451 & $.80(-3)$ & $-.21(3)$ & $-.33(1)$ & $.10(-1)$ & $.25(-2)$ & $-.10(-1)$ & $-.11(3)$ \\
\hline & & .160 & 91.9 & .622 & .431 & $.11(-2)$ & $-.57(2)$ & $-.25(1)$ & $.79(-2)$ & $.69(-2)$ & $-.45(-2)$ & $-.59(2)$ \\
\hline & & .185 & 91.2 & .622 & .411 & $.13(-2)$ & $+.22(2)$ & $-.21(1)$ & $\cdot 79(-2)$ & $\cdot 31(-2)$ & $-.88(-2)$ & $-.29(2)$ \\
\hline & & .214 & 92.6 & .592 & .388 & $.14(-2)$ & $.74(2)$ & $-.18(1)$ & $.94(-2)$ & $.14(-2)$ & $-.98(-2)$ & $-.82(1)$ \\
\hline & & .248 & 96.1 & .592 & .373 & $.15(-2)$ & $.12(3)$ & $-.18(1)$ & $.87(-2)$ & $.15(-2)$ & $-.11(-1)$ & $+.66(1)$ \\
\hline & & .273 & 99.9 & .592 & .365 & $.15(-2)$ & $.16(3)$ & $-.19(1)$ & $.82(-2)$ & $.79(-2)$ & $-.55(-2)$ & $.16(2)$ \\
\hline & & .301 & 106. & .622 & .368 & $.15(-2)$ & $.22(3)$ & $-.22(1)$ & $.66(-2)$ & $.86(-2)$ & $-.91(-2)$ & $.30(2)$ \\
\hline & \multirow[t]{6}{*}{1.2} & .103 & 94.8 & .568 & .402 & $.15(-2)$ & $.74(2)$ & $-.16(1)$ & $.96(-2)$ & $.44(-2)$ & $-.28(-2)$ & $.42(2)$ \\
\hline & & .119 & 93.6 & .568 & .384 & $.17(-2)$ & $.13(3)$ & $-.14(1)$ & $.10(-1)$ & $.59(-2)$ & $-.14(-2)$ & $.48(2)$ \\
\hline & & .138 & 99.2 & .541 & .364 & $.18(-2)$ & $.15(3)$ & $-.13(1)$ & $.12(-1)$ & $.40(-2)$ & $-.32(-2)$ & $.51(2)$ \\
\hline & & .160 & 103. & .541 & .350 & $.18(-2)$ & $.18(3)$ & $-.13(1)$ & $.13(-1)$ & $.63(-2)$ & $-.14(-2)$ & $.60(2)$ \\
\hline & & .185 & 108. & .515 & .333 & $.18(-2)$ & $.20(3)$ & $-.13(1)$ & $.14(-1)$ & $.32(-2)$ & $-.49(-2)$ & $.64(2)$ \\
\hline & & .214 & 115. & .515 & .214 & $.18(-2)$ & $.23(3)$ & $-.13(1)$ & $.14(-1)$ & $\cdot 71(-2)$ & $-.22(-2)$ & $.77(2)$ \\
\hline
\end{tabular}




\begin{tabular}{|c|c|c|c|c|c|c|c|c|c|c|c|c|}
\hline 9 & $\beta$ & $h$ & $\left(R_{p}\right)_{a}$ & $\alpha_{F}$ & $\mathrm{Tat}$ & $\begin{array}{l}\text { e. } \sigma= \\
\frac{\partial c_{r}}{\partial R_{p}}\end{array}$ & $\begin{array}{c}.5(10)^{4} \\
\frac{\partial R}{\partial n}\end{array}$ & $\begin{array}{l}\text { ont'd } \\
\frac{\partial R_{f}}{\partial m_{0}}\end{array}$ & $\frac{\partial R_{E}}{\partial m_{1}}$ & $\frac{\partial k_{p}}{\partial \sigma}$ & 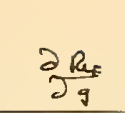 & $\frac{\partial R_{r}}{\partial \beta}$ \\
\hline & & .248 & 124. & .491 & .313 & $.17(-2)$ & $.26(3)$ & $-.14(1)$ & $.14(-1)$ & $.91(-3)$ & $-.11(-1)$ & $.85(2)$ \\
\hline & & .273 & 132. & .445 & .298 & $.16(-2)$ & $.26(3)$ &. $.14(1)$ & $.15(-1)$ & $-.27(-2)$ & $-.13(-1)$ & $.80(2)$ \\
\hline & & .301 & 141. & .445 & .302 & $.14(-2)$ & $.32(3)$ & $-.16(1)$ & $.13(-1)$ & $-.42(-2)$ & $-.19(-1)$ & $.97(2)$ \\
\hline & & .332 & 154. & .404 & .294 & $.12(-2)$ & $.36(3)$ & $-.18(1)$ & $.13(-1)$ & $-.82(-2)$ & $-.24(-1)$ & $.94(2)$ \\
\hline & & .366 & 170. & .404 & .309 & $.10(-2)$ & $.52(3)$ & $-.25(1)$ & $.90(-2)$ & $-.92(-2)$ & $-.37(-1)$ & $.12(3)$ \\
\hline & & .404 & 197. & .366 & .317 & $.76(-3)$ & $.75(3)$ & $-.35(1)$ & $.65(-2)$ &.$- .11(-1)$ & $-.48(-1)$ & $.13(3)$ \\
\hline & & .445 & 244. & .349 & .341 & $.47(-3)$ & $.14(4)$ & $-.64(1)$ & $.61(-3)$ & $-.20(-1)$ & $-.99(-1)$ & $.18(3)$ \\
\hline \multirow[t]{4}{*}{$0.5(10)^{5}$} & 0 & .00163 & 112. & 1.73 & .575 & $.37(-3)$ & $.29(5)$ & $.26(1)$ & $-.80(-1)$ &. $.54(-4)$ & $.83(-3)$ & $.46(1)$ \\
\hline & & .00241 & 142. & 1.42 & .555 & $.37(-3)$ & $.43(5)$ & $.30(1)$ & $-.93(-1)$ & $-.38(-4)$ & $.17(-2)$ & $.43(1)$ \\
\hline & & .00339 & 201. & 1.06 & .526 & $.29(-3)$ & $.70(5)$ & $.45(1)$ &.$- .14(0)$ & $-.22(-4)$ & $.36(-2)$ & $.46(1)$ \\
\hline & & .00454 & 313. & .719 & .493 & $.18(-3)$ & $.18(6)$ & $.88(1)$ & $-.28(0)$ & $-.12(-4)$ & $.10(-1)$ & $.52(1)$ \\
\hline $.5(10)^{5}$ & 0 & .00163 & 23.0 & 3.97 & .524 & $.30(-2)$ & $60(6)$ & $-.18(2)$ & $-.12(1)$ & $-.13(-2)$ & $-.84(-3)$ & $-.50(3)$ \\
\hline
\end{tabular}





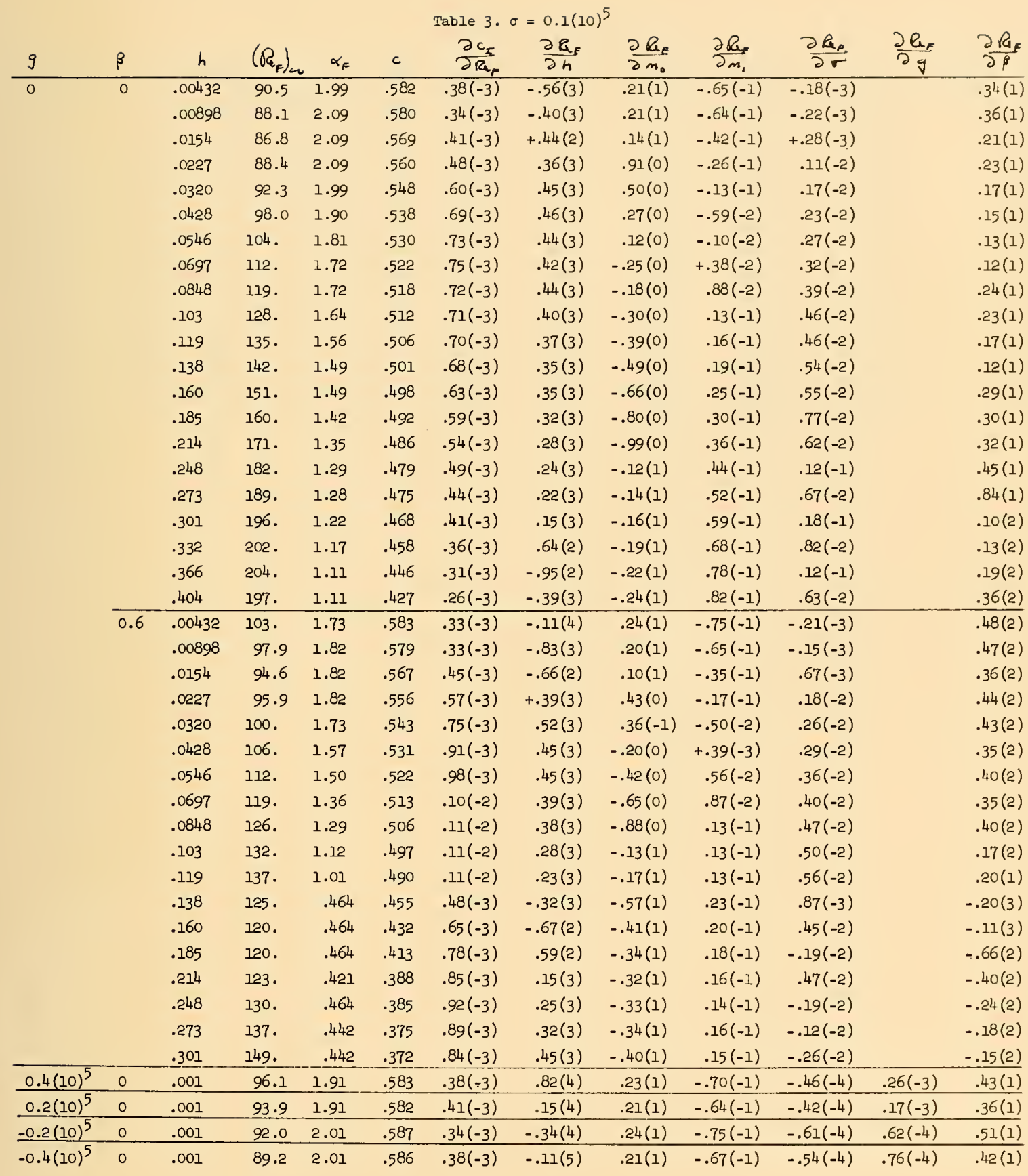





\begin{tabular}{|c|c|c|c|c|c|c|c|c|c|c|c|c|}
\hline 9 & $\beta$ & $h$ & $\left(r_{F}\right)_{a}$ & $\alpha_{F}$ & $c$ & $\frac{\partial c_{r}}{\partial \mathbb{R}_{r}}$ & $\frac{\partial R_{F}}{\partial h}$ & $\frac{\partial R_{F}}{\partial m_{*}}$ & $\frac{\partial a_{r}}{\partial m_{1}}$ & $\frac{\partial R_{E}}{\partial \sigma}$ & $\frac{\partial R_{r}}{\partial j}$ & $\frac{\partial \vec{a}}{\partial \beta}$ \\
\hline \multirow[t]{12}{*}{0} & 0 & .001 & 91.3 & 1.99 & .583 & $.36(-3)$ & & $.22(1)$ & $-.68(-1)$ & $-.45(-4)$ & & $.43(1)$ \\
\hline & & .00432 & 88.6 & 2.09 & .586 & $.38(-3)$ & $.66(3)$ & $.22(1)$ & $-.64(-1)$ & $.99(-4)$ & & $81(1)$ \\
\hline & & .00898 & 95.6 & 1.99 & .547 & $.54(-3)$ & $.19(4)$ & $.57(0)$ & $-.15(-1)$ & $.49(-3)$ & & $.46(1)$ \\
\hline & & .0154 & 108. & 1.81 & .530 & $.65(-3)$ & $.17(4)$ & $.15(0)$ & $-.18(-2)$ & $.73(-3)$ & & $.46(1)$ \\
\hline & & .0227 & 121. & 1.56 & .517 & $.70(-3)$ & $.22(4)$ & $.72(-3)$ & $.32(-2)$ & $.77(-3)$ & & $.34(1)$ \\
\hline & & .0319 & 135. & 1.49 & .509 & $.67(-3)$ & $.12(4)$ & $-.26(0)$ & .12(-1) & $.10(-2)$ & & $.45(1)$ \\
\hline & & .0428 & 149. & 1.42 & .502 & $.63(-3)$ & . $11(4)$ & $-.51(0)$ & .21(-1) & $.13(-2)$ & & $.56(1)$ \\
\hline & & .0546 & 163. & 1.35 & .497 & $.59(-3)$ & $.10(4)$ & $-.76(0)$ & $.29(-1)$ & $.15(-2)$ & & $.64(1)$ \\
\hline & & .0697 & 179. & 1.22 & .491 & $.55(-3)$ & $.88(3)$ & $-.97(0)$ & $.36(-1)$ & $.15(-2)$ & & $.56(1)$ \\
\hline & & .0848 & 194. & 1.17 & .486 & $.50(-3)$ & $.82(3)$ & $-.12(1)$ & $.45(-1)$ & $.20(-2)$ & & $.62(1)$ \\
\hline & & .103 & 210. & 1.11 & .482 & $.45(-3)$ & $.77(3)$ & $-.16(1)$ & $.57(-1)$ & $.19(-2)$ & & $.69(1)$ \\
\hline & & .119 & 224. & 1.06 & .478 & $.42(-3)$ & $.72(3)$ & $-.19(1)$ & $.67(-1)$ & $.30(-2)$ & & . $71(1)$ \\
\hline
\end{tabular}



Table 5. $\sigma=0.8(10)^{5}$

\begin{tabular}{|c|c|c|c|c|c|c|c|c|c|c|c|c|}
\hline 9 & $\beta$ & $h$ & $\left(\mathbb{R}_{p}\right)_{n}$ & $\alpha_{F}$ & $c$ & $\frac{\partial c_{I}}{\partial R_{p}}$ & $\frac{\partial R_{F}}{\partial h}$ & $\frac{\partial R_{x}}{\partial m_{0}}$ & $\frac{\partial R_{E}}{\partial m_{1}}$ & $\frac{\partial \mathbb{R}_{E}}{\partial \sigma}$ & $\frac{\partial G_{p}}{\partial g}$ & $\frac{\partial R_{F}}{\partial \beta}$ \\
\hline \multirow[t]{38}{*}{0} & \multirow[t]{21}{*}{0} & .001 & 89.8 & 1.99 & .577 & $.39(-3)$ & & $.19(1)$ & $-.58(-1)$ & $-.25(-4)$ & & $.35(1)$ \\
\hline & & .00432 & 95.1 & 1.99 & .548 & $.53(-3)$ & $.37(4)$ & $.64(0)$ & $-.17(-1)$ & $.22(-3)$ & $.20(-3)$ & $.49(1)$ \\
\hline & & .00899 & 112. & 1.72 & .525 & $.67(-3)$ & $.31(4)$ & $.96(-1)$ & $+.98(-4)$ & $\cdot 38(-3)$ & $.14(-3)$ & $.49(1)$ \\
\hline & & .0154 & 133. & 1.56 & .511 & $.65(-3)$ & $.26(4)$ & $-.26(0)$ & $.12(-1)$ & $.55(-3)$ & $.69(-4)$ & $.63(1)$ \\
\hline & & .0227 & 151. & 1.42 & .502 & $.62(-3)$ & $.22(4)$ & $-.55(0)$ & $.22(-1)$ & $.66(-3)$ & $-.36(-4)$ & .71(1) \\
\hline & & .0319 & 170. & 1.28 & .494 & $.57(-3)$ & $.18(4)$ & $-.85(0)$ & $.32(-1)$ & $.75(-3)$ & $-.19(-3)$ & $.76(1)$ \\
\hline & & .0428 & 190. & 1.17 & .489 & $.52(-3)$ & $.15(4)$ & $-.12(1)$ & $.42(-1)$ & $.83(-3)$ & $-.37(-3)$ & $.77(1)$ \\
\hline & & .0546 & 208. & 1.06 & .484 & $.47(-3)$ & $.13(4)$ &.$- .14(1)$ & $.51(-1)$ & $.87(-3)$ & $-.56(-3)$ & $.70(1)$ \\
\hline & & .0697 & 230. & 1.01 & .479 & $.42(-3)$ & $.12(4)$ & $-.19(1)$ & $.68(-1)$ & $.11(-2)$ & $-.56(-3)$ & $.86(1)$ \\
\hline & & .0848 & 250 . & .960 & .475 & $.37(-3)$ & .11(4) & $-.24(1)$ & $.84(-1)$ & $.12(-2)$ & $-.14(-2)$ & $.97(1)$ \\
\hline & & .103 & 272. & .870 & .471 & $.33(-3)$ & $.98(3)$ & $-.28(1)$ & $.97(-1)$ & $.11(-2)$ & $-.17(-2)$ & $.81(1)$ \\
\hline & & .119 & 290. & .829 & .468 & $\cdot 31(-3)$ & $.92(3)$ & $-.30(1)$ & $.11(0)$ & $.17(-2)$ & $+.12(-3)$ & $.85(1)$ \\
\hline & & .138 & 311. & .789 & .464 & $.26(-3)$ & $.87(3)$ & $-.39(1)$ & $.14(0)$ & $.13(-2)$ & $-.28(-2)$ & $.93(1)$ \\
\hline & & .160 & 334. & .752 & .460 & $.24(-3)$ & $.82(3)$ & $-.48(1)$ & $.16(0)$ & $.29(-2)$ & $+.39(-2)$ & $.11(2)$ \\
\hline & & .185 & 360. & .752 & .455 & $.19(-3)$ & $.78(3)$ & $-.62(1)$ & $.21(0)$ & $.15(-2)$ & $-.50(-2)$ & $.14(2)$ \\
\hline & & .214 & 388. & .682 & .449 & $.15(-3)$ & $.73(3)$ & $-.83(1)$ & $.27(0)$ & $.88(-2)$ & .31 (-1) & $.24(2)$ \\
\hline & & .248 & 422. & .648 & .443 & $.10(-3)$ & & $-.13(2)$ & $.44(0)$ & $.16(-2)$ & & $.34(2)$ \\
\hline & & .260 & 434. & .623 & .440 & $.90(-4)$ & $.68(3)$ & $-.15(2)$ & $.50(0)$ & $\cdot 30(-2)$ & $-.75(-2)$ & $.37(2)$ \\
\hline & & .273 & 447. & .611 & .437 & $.78(-4)$ & $.66(3)$ & $-.17(2)$ & $.60(0)$ & $.15(0)$ & $.81(0)$ & $.50(2)$ \\
\hline & & .287 & 465. & .587 &. .434 & $.55(-4)$ & $.78(3)$ & $-.26(2)$ & $.87(0)$ & $.67(-2)$ & $.31(-3)$ & $.62(2)$ \\
\hline & & .301 & 486. & .576 & .433 & $.39(-4)$ & $.11(4)$ & $-.36(2)$ & $.13(1)$ & $.11(1)$ & $.59(1)$ & $.12(3)$ \\
\hline & 0.2 & .001 & 91.4 & 1.99 & .579 & $.35(-3)$ & & $.21(1)$ & $-.64(-1)$ & $-.30(-4)$ & & $.23(2)$ \\
\hline & 0.4 & .001 & 96.3 & 1.90 & .579 & $.32(-3)$ & & $.23(1)$ & $-.70(-1)$ & $-.33(-4)$ & & $.43(2)$ \\
\hline & \multirow[t]{15}{*}{0.6} & .001 & 104. & 1.72 & .577 & $.33(-3)$ & & $.22(1)$ & $-.69(-1)$ & $-.30(-4)$ & & $.49(2)$ \\
\hline & & .00432 & 110. & 1.72 & .548 & $.47(-3)$ & $.44(4)$ & $.65(0)$ & $-.18(-1)$ & $.30(-3)$ & $.19(-3)$ & $.68(2)$ \\
\hline & & .00898 & 130. & 1.49 & .524 & $.61(-3)$ & $.36(4)$ & $-.88(-2)$ & $+.23(-2)$ & $.47(-3)$ & $.12(-3)$ & $.70(2)$ \\
\hline & & .0154 & 152 . & 1.29 & .509 & $.63(-3)$ & $.27(4)$ & $-.40(0)$ & $.14(-1)$ & $.59(-3)$ & $.44(-4)$ & $.76(2)$ \\
\hline & & .0227 & 173 . & 1.17 & .500 & $.60(-3)$ & $.23(4)$ & $-.78(0)$ & $.26(-1)$ & $.73(-3)$ & $-.76(-4)$ & $.89(2)$ \\
\hline & & .0320 & 194. & 1.06 & .492 & $.56(-3)$ & $.19(4)$ & $-.12(1)$ & $\cdot 38(-1)$ & $.86(-3)$ & $-.26(-3)$ & $.10(3)$ \\
\hline & & .0428 & 214. & .960 & .486 & $.52(-3)$ & $.16(4)$ & $-.17(1)$ & $.50(-1)$ & $.97(-3)$ & $-.50(-3)$ & $.11(3)$ \\
\hline & & .0546 & 234. & .870 & .481 & $.48(-3)$ & $.14(4)$ & $-.22(1)$ & $.62(-1)$ & $.11(-2)$ & $-.74(-3)$ & $.11(3)$ \\
\hline & & .0697 & 256. & .789 & .476 & $.45(-3)$ & $.13(4)$ & $-.28(1)$ & $.75(-1)$ & $.12(-2)$ & $-.12(-2)$ & $.11(3)$ \\
\hline & & .0848 & 276. & .716 & .472 & $.42(-3)$ & $.11(4)$ & $-.34(1)$ & $.86(-1)$ & $.13(-2)$ & $-.15(-2)$ & $.10(3)$ \\
\hline & & .103 & 299. & .650 & .468 & $\cdot 38(-3)$ & $.11(4)$ & $-.42(1)$ & $.10(0)$ & $.14(-2)$ & $-.25(-2)$ & $.97(2)$ \\
\hline & & .119 & 318. & .619 & .464 & $.35(-3)$ & $.11(4)$ & $-.50(1)$ & $.12(0)$ & $.19(-2)$ & $-.15(-2)$ & $.11(3)$ \\
\hline & & .138 & 340. & .561 & .460 & $.33(-3)$ & $.10(4)$ & $-.60(1)$ & $.13(0)$ & $.17(-2)$ & $-.33(-2)$ & $.94(2)$ \\
\hline & & .160 & 365. & .485 & .457 & $.31(-3)$ & $.10(4)$ & $-.75(1)$ & $.13(0)$ & $.20(-2)$ & $-.47(-2)$ & $.40(2)$ \\
\hline & & .185 & 343. & 1.92 & .431 & $.17(-3)$ & $.10(4)$ & $-.20(2)$ & $.10(0)$ & $.27(-2)$ & $-.14(-1)$ & $-.68(3)$ \\
\hline
\end{tabular}



Table 6. $\sigma=0.12(10)^{7}$

\begin{tabular}{|c|c|c|c|c|c|c|c|c|c|c|c|c|}
\hline 9 & $\beta$ & $h$ & $\left(\mathbb{R}_{F}\right)_{\omega}$ & $\alpha_{F}$ & $c$ & $\frac{\partial c_{I}}{\partial A_{p}}$ & $\frac{\partial R_{F}}{\partial h}$ & $\frac{\partial R_{k}}{\partial m_{0}}$ & $\frac{\partial G_{e}}{\partial m_{1}}$ & $\frac{\partial G_{F}}{\partial \sigma}$ & $\frac{\partial R_{r}}{\partial g}$ & $\frac{\partial a_{p}}{\partial p}$ \\
\hline \multirow[t]{11}{*}{0} & \multirow[t]{11}{*}{0} & .0001 & 88.9 & 2.04 & .573 & $.38(-3)$ & $-.94(4)$ & $.18(1)$ & $-.54(-1)$ & $-.87(-6)$ & $.12(-4)$ & $.42(1)$ \\
\hline & & .000161 & 89.40 & 2.040 & .5639 & $.43(-3)$ & $.25(5)$ & $.13(1)$ & $-.38(-1)$ & $.38(-5)$ & $.14(-4)$ & $.42(1)$ \\
\hline & & .000259 & 93.62 & 1.981 & .5508 & $.53(-3)$ & $.49(5)$ & $.74(0)$ & $-.21(-1)$ & $.12(-4)$ & $.13(-4)$ & $.46(1)$ \\
\hline & & .000418 & 102.6 & 1.867 & .5366 & $.61(-3)$ & $.53(5)$ & $.34(0)$ & $-.79(-2)$ & $.20(-4)$ & $.11(-4)$ & $.52(1)$ \\
\hline & & .000673 & 116.3 & 1.708 & .5228 & $.65(-3)$ & $.46(5)$ & $.34(-1)$ & $.23(-2)$ & $.28(-4)$ & $.75(-5)$ & $.60(1)$ \\
\hline & & .001 & 131. & 1.54 & .512 & $.66(-3)$ & $.37(5)$ & $-.20(0)$ & $.10(-1)$ & $.34(-4)$ & $.34(-5)$ & $.65(1)$ \\
\hline & & .002 & 164. & 1.28 & .497 & $.59(-3)$ & $.24(5)$ & $-.67(0)$ & $.27(-1)$ & $.45(-4)$ & $-.82(-5)$ & $.81(1)$ \\
\hline & & .004 & 207. & 1.05 & .486 & $.48(-3)$ & $.16(5)$ & $-.14(1)$ & $.52(-1)$ & $.58(-4)$ & $-.46(-4)$ & $.10(2)$ \\
\hline & & .007 & 251. & .880 & .479 & $.40(-3)$ & $.11(5)$ & $-.22(1)$ & $.78(-1)$ & $.68(-4)$ & $-.11(-3)$ & $.12(2)$ \\
\hline & & .015 & 324. & .692 & .472 & $.30(-3)$ & $.65(4)$ & $-.36(i)$ & $.13(0)$ & $.84(-4)$ & $-.31(-3)$ & $.15(2)$ \\
\hline & & .030 & 415. & .562 & .465 & $.22(-3)$ & $.45(4)$ & $-.56(1)$ & $.19(0)$ & $.11(-3)$ & $-.74(-3)$ & $.18(2)$ \\
\hline \multirow[t]{32}{*}{$-0.13(10)^{5}$} & \multirow[t]{20}{*}{0} & .0001 & 88.9 & 2.04 & .573 & $.38(-3)$ & $-.94(4)$ & $.18(1)$ & $-.54(-1)$ & $-.87(-6)$ & $.12(-4)$ & $.42(1)$ \\
\hline & & .000161 & 89.40 & 2.040 & .5639 & $.43(-3)$ & $.25(5)$ & $.13(1)$ & $-.38(-1)$ & $.38(-5)$ & $.14(-4)$ & $.42(1)$ \\
\hline & & .000259 & 93.62 & 1.981 & .5508 & $.53(-3)$ & $.49(5)$ & $.74(0)$ & $-.21(-1)$ & $.12(-4)$ & $.13(-4)$ & $.46(1)$ \\
\hline & & .000418 & 102.6 & 1.867 & .5366 & $.61(-3)$ & $.53(5)$ & $.34+(0)$ & $-.79(-2)$ & $.20(-4)$ & $.11(-4)$ & $.52(1)$ \\
\hline & & .000673 & 116.3 & 1.708 & .5228 & $.65(-3)$ & $.46(5)$ & $.34(-1)$ & $.23(-2)$ & $.28(-4)$ & $.75(-5)$ & $.60(1)$ \\
\hline & & .001 & 131. & 1.54 & .512 & $.66(-3)$ & $.37(5)$ & $-.20(0)$ & $.10(-1)$ & $.34(-4)$ & $.34(-5)$ & $.65(1)$ \\
\hline & & .002 & 164. & 1.28 & .497 & $.59(-3)$ & $.25(5)$ & $-.72(0)$ & $.28(-1)$ & $.45(-4)$ & $-.95(-5)$ & $.81(1)$ \\
\hline & & .004 & 210. & 1.03 & .486 & $.48(-3)$ & $.18(5)$ & $-.16(1)$ & $.57(-1)$ & $.59(-4)$ & $-.36(-3)$ & $.10(2)$ \\
\hline & & .007 & 274. & .796 & .482 & $.34(-3)$ & $.24(5)$ & $-.37(1)$ & $.12(0)$ & $.73(-4)$ & $-.41(-2)$ & . $11(2)$ \\
\hline & & .010 & 416. & .556 & .488 & $.14(-3)$ & $.87(5)$ & $-.13(2)$ & $.44(0)$ & $.77(-4)$ & $-.35(-1)$ & $.71(1)$ \\
\hline & & .011 & 529. & .448 & .489 & $.89(-4)$ & $.14(6)$ & $-.23(2)$ & $.78(0)$ & $.62(-4)$ & $-.67(-1)$ & $.72(0)$ \\
\hline & & .012 & 700. & .340 & .487 & $.57(-4)$ & $.20(6)$ & $-.38(2)$ & $.13(1)$ & $.38(-4)$ & $-.11(0)$ & $-.59(1)$ \\
\hline & & .013 & 924. & .256 & .485 & $.41(-4)$ & $.24(6)$ & $-.54(2)$ & $.18(1)$ & $.17(-4)$ & $-.14(0)$ & $-.97(1)$ \\
\hline & & .014 & 1190. & .198 & .483 & . $31(-4)$ & $.28(6)$ & $-.68(2)$ & $.23(1)$ & $.14(-4)$ & $-.17(0)$ & $-.11(2)$ \\
\hline & & .015 & 1500 . & .158 & .483 & $.24(-4)$ & $.32(6)$ & $-.91(2)$ & $.30(1)$ & $.40(-5)$ & $-.23(0)$ & $-.14(2)$ \\
\hline & & .016 & 1840. & .127 & .482 & $.20(-4)$ & $.36(6)$ & $-.11(3)$ & $.37(1)$ & $.94(-5)$ & $-.28(0)$ & $-.16(2)$ \\
\hline & & .017 & 2240 & .105 & .481 & $.16(-4)$ & $.4 i(6)$ & $-.14(3)$ & $.46(1)$ & $-.11(-5)$ & $-.33(0)$ & $-.19(2)$ \\
\hline & & .018 & 2680. & .0877 & .481 & $.14(-4)$ & $.46(6)$ & $-.17(3)$ & $.55(1)$ & $.27(-5)$ & $-.40(0)$ & $-.22(2)$ \\
\hline & & .019 & 3170. & .0742 & .481 & $.21(-4)$ & $.52(6)$ & $-.20(3)$ & $.66(1)$ & $.48(-5)$ & $-.48(0)$ & $-.26(2)$ \\
\hline & & .020 & 3720. & .0629 & .480 & $.98(-5)$ & $.56(6)$ & $-.23(3)$ & $.76(1)$ & $.13(-4)$ & $-.54(0)$ & $-.32(2)$ \\
\hline & 1.0 & .0130 & 875. & .265 & .487 & $.51(-4)$ & $.22(6)$ & $-.44(2)$ & $.14(1)$ & $.72(-4)$ & $-.13(0)$ & $-.90(1)$ \\
\hline & 1.1 & & 874. & .265 & .487 & $.52(-4)$ & $.21(6)$ & $-.42(2)$ & $.14(1)$ & $.83(-4)$ & $-.12(0)$ & $+.17(2)$ \\
\hline & 1.0 & .0131 & 896. & .257 & .487 & $.49(-4)$ & $.22(6)$ & $-.45(2)$ & $.15(1)$ & $.69(-4)$ & $-.13(0)$ & $-.19(2)$ \\
\hline & 1.1 & & 894. & .257 & .487 & $.51(-4)$ & $.21(6)$ & $-.43(2)$ & $.14(1)$ & $.81(-4)$ & $-.12(0)$ & $+.59(1)$ \\
\hline & 1.1 & .0134 & 960. & .239 & .486 & $.46(-4)$ & $.23(6)$ & $-.49(2)$ & $.16(1)$ & $.69(-4)$ & $-.14(0)$ & $-.22(2)$ \\
\hline & 1.2 & & 958. & .239 & .487 & $.47(-4)$ & $.22(6)$ & $-.47(2)$ & $.15(1)$ & $.80(-4)$ & $-.13(0)$ & $+.28(1)$ \\
\hline & 1.4 & .0140 & 1090. & .208 & .486 & $.41(-4)$ & $.24(6)$ & $-.55(2)$ & $.18(1)$ & $.88(-4)$ & $-.16(0)$ & $-.35(1)$ \\
\hline & 1.5 & & 1090. & .208 & .486 & $.42(-4)$ & $.24(6)$ & $-.53(2)$ & $.17(1)$ & $.97(-4)$ & $-.15(0)$ & $+.22(2)$ \\
\hline & 1.8 & .0150 & 1350. & .166 & .486 & $.34(-4)$ & $.28(6)$ & $-.67(2)$ & $.22(1)$ & $.97(-4)$ & $-.19(0)$ & $+.23(0)$ \\
\hline & 1.9 & & 1350. & .166 & .486 & $.35(-4)$ & $.27(6)$ & $-.66(2)$ & $.21(1)$ & $.12(-3)$ & $-.18(0)$ & $.22(2)$ \\
\hline & 2.2 & .0160 & 1640 & .137 & .485 & $.27(-4)$ & $.32(6)$ & $-.85(2)$ & $.27(1)$ & $.12(-3)$ & $-.24(0)$ & $.12(1)$ \\
\hline & 2.3 & & 1640 & .135 & .485 & $.29(-4)$ & $.31(6)$ & $-.81(2)$ & $.25(1)$ & $.13(-3)$ & $-.22(0)$ & 70 \\
\hline
\end{tabular}



Table 7. $\sigma=0.23(10)^{7}$

\begin{tabular}{|c|c|c|c|c|c|c|c|c|c|c|c|c|}
\hline 9 & $\beta$ & $h$ & $\left(G_{F}\right)_{L}$ & $\alpha_{F}$ & c & $\frac{\partial c_{I}}{\partial R_{p}}$ & $\frac{\partial \mu_{f}}{\partial h}$ & $\frac{\partial R_{E}}{\partial m_{0}}$ & $\frac{\partial \mathfrak{a}_{F}}{\partial m_{1}}$ & $\frac{\partial a_{E}}{\partial \sigma}$ & $\frac{\partial R_{f}}{\partial j}$ & $\begin{array}{l}\partial R_{p} \\
\partial \beta\end{array}$ \\
\hline \multirow[t]{13}{*}{0} & \multirow[t]{13}{*}{0} & .0001 & 90.4 & 2.03 & .560 & $.46(-3)$ & $.71(5)$ & $.11(1)$ & $-.32(-1)$ & $.34(-5)$ & $.84(-5)$ & $.44(1)$ \\
\hline & & .000161 & 96.4 & 1.95 & .546 & $.55(-3)$ & $.10(6)$ & $.59(0)$ & $-.16(-1)$ & $.78(-5)$ & $.72(-5)$ & $.49(1)$ \\
\hline & & .000259 & 107. & 1.79 & .531 & $.65(-3)$ & $.92(5)$ & $.24(0)$ & $-.44(-2)$ & $.11(-4)$ & $.54(-5)$ & $.51(1)$ \\
\hline & & .000418 & 122. & 1.63 & .518 & $.66(-3)$ & $.79(5)$ & $-.64(-1)$ & $.56(-2)$ & $.16(-4)$ & $.33(-5)$ & $.62(1)$ \\
\hline & & .000673 & 142. & 1.41 & .506 & $.66(-3)$ & $.57(5)$ & $-.32(0)$ & $.14(-1)$ & $.18(-4)$ & $.71(-6)$ & $.64(1)$ \\
\hline & & .001 & 162. & 1.29 & .498 & $.60(-3)$ & $.47(5)$ & $-.64(0)$ & $+.25(-1)$ & $.23(-4)$ & $-.33(-5)$ & $.79(1)$ \\
\hline & & .002 & 204. & 1.06 & .486 & $.49(-3)$ & $.30(5)$ & $-.13(1)$ & $.49(-1)$ & $.29(-4)$ & $-.21(-4)$ & $.10(2)$ \\
\hline & & .004 & 257. & .854 & .479 & $.39(-3)$ & $.19(5)$ & $-.23(1)$ & $.82(-1)$ & $.36(-4)$ & $-.66(-4)$ & $.13(2)$ \\
\hline & & .005 & 277. & .797 & .477 & $.36(-3)$ & $.16(5)$ & $-.27(1)$ & $.95(-1)$ & $.38(-4)$ & $-.90(-4)$ & $.13(2)$ \\
\hline & & .006 & 294. & .749 & .475 & $.33(-3)$ & $.14(5)$ & $-.29(1)$ & $.10(0)$ & $.38(-4)$ & $-.11(-3)$ & $.13(2)$ \\
\hline & & .008 & 323. & .690 & .473 & $.30(-3)$ & $.12(5)$ & $-.36(1)$ & $.13(0)$ & $.44(-4)$ & $-.17(-3)$ & $.15(2)$ \\
\hline & & .010 & 348. & .644 & .471 & $.27(-3)$ & $.10(5)$ & $-.41(1)$ & $.14(0)$ & $.46(-4)$ & $-.22(-3)$ & $.16(2)$ \\
\hline & & .014 & 389. & .583 & .468 & $.24(-3)$ & $.83(4)$ & $-.50(1)$ & $.17(0)$ & $.52(-4)$ & $-.34(-3)$ & $.18(2)$ \\
\hline \multirow[t]{36}{*}{$-0.36(10)^{5}$} & & .000161 & 96.4 & 1.95 & .546 & $.55(-3)$ & $.10(6)$ & $.59(0)$ & $-.16(-1)$ & $.78(-5)$ & $.72(-5)$ & $.49(1)$ \\
\hline & & .000259 & 107. & 1.79 & .531 & $.65(-3)$ & $.92(5)$ & $.24(0)$ & $-.44(-2)$ & $.11(-4)$ & $.54(-5)$ & $.51(1)$ \\
\hline & & .000418 & 122. & 1.63 & .518 & $.66(-3)$ & $.79(5)$ & $-.64(-1)$ & $.56(-2)$ & $.16(-4)$ & $.33(-5)$ & $.62(1)$ \\
\hline & & .000673 & 142. & 1.41 & .506 & $.66(-3)$ & $.57(5)$ & $-.32(0)$ & $.14(-1)$ & $.18(-4)$ & $.71(-6)$ & $.64(1)$ \\
\hline & & .001 & 162. & 1.29 & .498 & $.60(-3)$ & $.49(5)$ & $-.68(0)$ & $.26(-1)$ & $.23(-4)$ & $-.23(-5)$ & $.81(1)$ \\
\hline & & .002 & 206. & 1.04 & .487 & $.49(-3)$ & $.35(5)$ & $-.15(1)$ & $.54(-1)$ & $.29(-4)$ & $-.11(-3)$ & $.99(1)$ \\
\hline & & .004 & 297. & .743 & .484 & $.29(-3)$ & $.65(5)$ & $-.48(1)$ & $.17(0)$ & $.40(-4)$ & $-.27(-2)$ & $.12(2)$ \\
\hline & & .005 & 399. & .579 & .489 & $.16(-3)$ & $.16(6)$ & $-.12(2)$ & $.40(0)$ & $.41(-4)$ & $-.11(-1)$ & $.81(1)$ \\
\hline & & .0051 & 416. & .560 & .489 & $.14(-3)$ & $.18(6)$ & $-.13(2)$ & $.45(0)$ & $.40(-4)$ & $-.13(-1)$ & $.72(1)$ \\
\hline & & .0052 & 434. & .540 & .489 & $.13(-3)$ & $.20(6)$ & $-.15(2)$ & $.50(0)$ & $\cdot 39(-4)$ & $-.15(-1)$ & $.62(1)$ \\
\hline & & .0053 & 455. & .515 & .489 & $.12(-3)$ & $.22(6)$ & $-.17(2)$ & $.56(0)$ & $\cdot 37(-4)$ & $-.17(-1)$ & $.49(1)$ \\
\hline & & .0054 & 477. & .495 & .490 & $.11(-3)$ & $.24(6)$ & $-.19(2)$ & $.63(0)$ & $\cdot 35(-4)$ & $-.19(-1)$ & $.36(1)$ \\
\hline & & .0055 & 502. & .470 & .489 & $.98(-4)$ & $.26(6)$ & $-.21(2)$ & $.70(0)$ & $.33(-4)$ & $-.21(-1)$ & $.22(1)$ \\
\hline & & .0056 & 529. & .450 & .489 & $.88(-4)$ & $.29(6)$ & $-.24(2)$ & $.80(0)$ & $.32(-4)$ & $-.25(-1)$ & $.93(0)$ \\
\hline & & .0057 & 558. & .426 & .489 & $.81(-4)$ & $\cdot 31(6)$ & $-.26(2)$ & $.87(0)$ & $.29(-4)$ & $-.27(-1)$ & $-.53(0)$ \\
\hline & & .0058 & 590. & .404 & .489 & $.73(-4)$ & $.34(6)$ & $-.29(2)$ & $.97(0)$ & $.26(-4)$ & $-.30(-1)$ & $-.19(1)$ \\
\hline & & .0059 & 624. & .384 & .488 & $.66(-4)$ & $.36(6)$ & $-.34(2)$ & . $11(1)$ & $.25(-4)$ & $-.35(-1)$ & $-.32(1)$ \\
\hline & & .0060 & 660. & .360 & .488 & $.62(-4)$ & $.38(6)$ & $-.35(2)$ & $.12(1)$ & $.21(-4)$ & $-.36(-1)$ & $-.42(1)$ \\
\hline & & .0062 & 739. & .324 & .487 & $.52(-4)$ & $.42(6)$ & $-.42(2)$ & $.14(1)$ & $.16(-4)$ & $-.42(-1)$ & $-.61(1)$ \\
\hline & & .0064 & 826. & .291 & .486 & $.45(-4)$ & $.43(6)$ & $-.45(2)$ & $.15(1)$ & $.12(-4)$ & $-.45(-1)$ & $-.69(1)$ \\
\hline & & .0066 & 919. & .258 & .485 & $.41(-4)$ & $.48(6)$ & $-.54(2)$ & $.18(1)$ & $.10(-4)$ & $-.52(-1)$ & $-.80(1)$ \\
\hline & & .0068 & 1020 & .234 & .485 & $.36(-4)$ & $.53(6)$ & $-.62(2)$ & $.21(1)$ & $.72(-5)$ & $-.60(-1)$ & $-.88(1)$ \\
\hline & & .0070 & 1120. & .210 & .484 & $.33(-4)$ & $.54(6)$ & $-.66(2)$ & $.22(1)$ & $.51(-5)$ & $-.63(-1)$ & $-.89(1)$ \\
\hline & & .00720 & 1240. & .192 & .483 & $.30(-4)$ & $.58(6)$ & $-.75(2)$ & $.25(1)$ & $.40(-5)$ & $-.70(-1)$ & $-.93(1)$ \\
\hline & & .00740 & 1350. & .175 & .483 & $.27(-4)$ & $.62(6)$ & $-.83(2)$ & $.28(1)$ & $.45(-5)$ & $-.76(-1)$ & $-.95(1)$ \\
\hline & & .00750 & 1410. & .167 & .483 & $.26(-4)$ & $.62(6)$ & $-.85(2)$ & $.29(1)$ & $.35(-5)$ & $-.78(-1)$ & $-.96(1)$ \\
\hline & & .00770 & 1540. & .153 & .482 & $.24(-4)$ & $.65(6)$ & $-.93(2)$ & .31(1) & $.16(-5)$ & $-.85(-1)$ & $-.99(1)$ \\
\hline & & .00790 & 1680. & .140 & .482 & $.23(-4)$ & $.67(6)$ & $-.99(2)$ & $.33(1)$ & $.24(-5)$ & $-.89(-1)$ & $-.10(2)$ \\
\hline & & .00810 & 1820. & .129 & .482 & $.21(-4)$ & $.70(6)$ & $-.11(3)$ & $.36(1)$ & $.59(-6)$ & $-.97(-1)$ & $-.10(2)$ \\
\hline & & .00800 & 6460. & .520 & .257 & $.11(-5)$ & $-.58(6)$ & $.68(3)$ & $-.20(2)$ & $.89(-5)$ & $.91(-1)$ & $.24(3)$ \\
\hline & & .0100 & 652. & 1.92 & .365 & $.14(-3)$ & $-.33(4)$ & $-.11(1)$ & $-.68(-1)$ & $-16(-3)$ & $-.23(-2)$ & $-.17(2)$ \\
\hline & .1 & .0060 & 660. & .360 & .488 & $.62(-4)$ & $.37(6)$ & $-.34(2)$ & $.12(1)$ & $.21(-4)$ & $-.35(-1)$ & $-.12(2)$ \\
\hline & .2 & & 658. & .364 & .488 & $.62(-4)$ & $.38(6)$ & $-.35(2)$ & $.12(1)$ & $.22(-4)$ & $-.36(-1)$ & $-.18(2)$ \\
\hline & .3 & & 657. & .364 & .488 & $.63(-4)$ & $.38(6)$ & $-.34(2)$ & $.11(1)$ & $.25(-4)$ & $-.35(-1)$ & $-.19(2)$ \\
\hline & .4 & & 655. & .364 & .488 & $.65(-4)$ & $.37(6)$ & $-.33(2)$ & $.11(1)$ & $.27(-4)$ & $-.34(-1)$ &.$- .15(2)$ \\
\hline & .5 & & 653. & .364 & .489 & $.67(-4)$ & $.36(6)$ & $-.32(2)$ & $.11(1)$ & $\cdot 30(-4)$ & $-.33(-1)$ & $-.55(1)$ \\
\hline
\end{tabular}



Table 7. $\sigma=0.23(10)^{\prime}$ Cont'd

\begin{tabular}{|c|c|c|c|c|c|c|c|c|c|c|c|c|}
\hline 9 & $\beta$ & $h$ & $\left(R_{F}\right)_{\mu}$ & $\alpha_{F}$ & c & $\frac{\partial c_{k}}{\partial A_{E}}$ & $\frac{\partial \varepsilon_{E}}{\partial h}$ & $\frac{\partial a_{1}}{\partial m_{1}}$ & $\frac{\partial R_{F}}{\partial m_{1}}$ & $\frac{\partial \varepsilon_{F}}{\partial \sigma}$ & $\frac{\partial G_{p}}{\partial g}$ & $\frac{\partial k a_{E}}{\partial \beta}$ \\
\hline & .6 & & 653. & .360 & .488 & $.70(-4)$ & $.33(6)$ & $-.30(2)$ & $.10(1)$ & $.34(-4)$ & $-.32(-1)$ & $+.92(1)$ \\
\hline & .5 & .0061 & 689. & .342 & .488 & $.62(-4)$ & $.37(6)$ & $-.34(2)$ & $.11(1)$ & $.26(-4)$ & $-.36(-1)$ & $-.19(2)$ \\
\hline & .6 & & 687. & .343 & .488 & $.64(-4)$ & $.36(6)$ & $-.33(2)$ & $.11(1)$ & $.31(-4)$ & $-.34(-1)$ & $-.60(1)$ \\
\hline & .7 & & 687. & .343 & .488 & $.66(-4)$ & $.35(6)$ & $-.32(2)$ & $.11(1)$ & $.35(-4)$ & $-.33(-2)$ & $+.13(2)$ \\
\hline & .6 & .0062 & 724. & .326 & .488 & $.59(-4)$ & $.39(6)$ & $-.37(2)$ & $.12(2)$ & $.28(-4)$ & $-.38(-1)$ & $-.20(2)$ \\
\hline & .7 & & 722. & .326 & .488 & $.61(-4)$ & $.37(6)$ & $-.35(2)$ & $.12(1)$ & $.33(-4)$ & $-.36(-1)$ & $-.42(1)$ \\
\hline & .8 & & 722. & .326 & .488 & $.63(-4)$ & $.36(6)$ & $-.34(2)$ & $.21(1)$ & $.37(-4)$ & $-.35(-1)$ & $+.17(2)$ \\
\hline & .9 & .0065 & 836. & .280 & .488 & $.52(-4)$ & $.43(6)$ & $-.41(2)$ & $.14(1)$ & $.32(-4)$ & $-.42(-1)$ & $-.15(2)$ \\
\hline & 2.0 & & 834. & .280 & .488 & $.54(-4)$ & $.40(6)$ & $-.40(2)$ & $.13(1)$ & $.38(-4)$ & $-.42(-1)$ & $+.76(1)$ \\
\hline & 1.2 & .00675 & 937. & .250 & .488 & $.48(-4)$ & $.44(6)$ & $-.46(2)$ & $.15(1)$ & $.43(-4)$ & $-.47(-1)$ & $.10(2)$ \\
\hline & 2.3 & & 937. & .248 & .488 & $.50(-4)$ & $.42(6)$ & $-.43(2)$ & $.14(1)$ & $.48(-4)$ & $-.44(-1)$ & $.35(2)$ \\
\hline & 2.4 & .0070 & 1050. & .223 & .487 & $.43(-4)$ & $.48(6)$ & $-.51(2)$ & $.17(1)$ & $.47(-4)$ & $-.53(-1)$ & $.12(2)$ \\
\hline & 1.5 & & 1050. & .221 & .487 & $.45(-4)$ & $.45(6)$ & $-.48(2)$ & $.16(1)$ & $.53(-4)$ & $-.50(-1)$ & $.35(2)$ \\
\hline
\end{tabular}




